Supporting Information for

\title{
Me2AISEt-Promoted Domino Dieckmann Cyclization Enables the Total Synthesis of Polycyclic Polyprenylated Acylphloroglucinols
}

\author{
Liping Wang, ${ }^{\dagger}$ Lian Sun, ${ }^{\dagger}$ Xueying Wang, Rong Wu, Hua Zhou, Changwu Zheng* \\ and Hongxi $\mathrm{Xu}^{*}$ \\ Email: zhengcw@shutcm.edu.cn (CWZ),xuhongxi88@gmail.com (HXX).
}

\section{Contents}

General Information S2

Scalable synthesis of the functionalized linear precursors $14 \mathrm{a}$ and $\mathbf{1 4 b}$ S2

Scalable synthesis of the functionalized linear precursors $14 \mathrm{c}, 14 \mathrm{~d}, 14 \mathrm{e}, 14 \mathrm{f}$. S5

Table S1. Evaluation of the domino Dieckmann cyclization with 14a S9

Procedure for the base-catalyzed cascade Dieckmann condensation with 14a S10

General procedure for the $\mathrm{Me}_{3} \mathrm{Al} / \mathrm{EtSH}$ catalyzed cascade Dieckmann condensation

${ }^{1} \mathrm{H} \mathrm{NMR}$ analysis of the mixed $\mathrm{Me}_{3} \mathrm{Al}$ and $\mathrm{EtSH}$ performed in $\mathrm{CD}_{2} \mathrm{Cl}_{2}$

Completion of total synthesis of epi-clusianone, garcimultiflorone $\mathrm{H}$, garcimultiflorone I, guttiferone I, oblongifolin A, oblongifolin D and anologues

NMR spectroscopic comparison between synthetic and natural products

MTT assay of cell viability and Cytotoxicity data

References 


\section{General Information}

All glassware was dried with a hot air gun and all reactions were carried out under an atmosphere of dry $\mathrm{N}_{2}$ unless otherwise stated. All reagents and dry solvents were used as received from the supplier. Melting points were performed using a Gallenkamp melting point apparatus and are uncorrected. IR spectra were carried out on either a neat oil or a solid using a Perkin-Elmer Spectrum $983 \mathrm{G}$ instrument. Wavelengths $(v)$ are reported in $\mathrm{cm}^{-1}$. ${ }^{1} \mathrm{H}$ and ${ }^{13} \mathrm{C}$ NMR were performed on Bruker AV-400, AV-600 or Agilent 400 NMR spectrometers in $\mathrm{CDCl}_{3}, \mathrm{CD}_{3} \mathrm{OD}$ or DMSO- $d_{6}$. Chemical shifts $(\delta)$ are quoted in ppm, coupling constants $(J)$ in Hz. All spectra are calibrated based on the solvent peak used unless otherwise stated. The following abbreviations apply: (br) broad, (s) singlet, (d) doublet, (t) triplet, (q) quartet and (m) multiplet. Mass spectrometry was performed on a SYNAPT G2-Si HDMS (Waters Corp., Manchester, UK). A Waters 2535 series machine equipped with an Xbridge $\mathrm{C}_{18}$ column $(4.6 \times 250 \mathrm{~mm}, 5 \mathrm{~mm})$ was used for HPLC analysis, and a preparative Xbridge Prep $\mathrm{C}_{18}$ OBD column $(19 \times 250 \mathrm{~mm}, 5 \mathrm{~mm})$ was used for the sample preparation. Flash column chromatography was performed using silica gel (300-400 mesh).

\section{Scalable synthesis of the functionalized linear precursors $14 a$ and $14 b$}

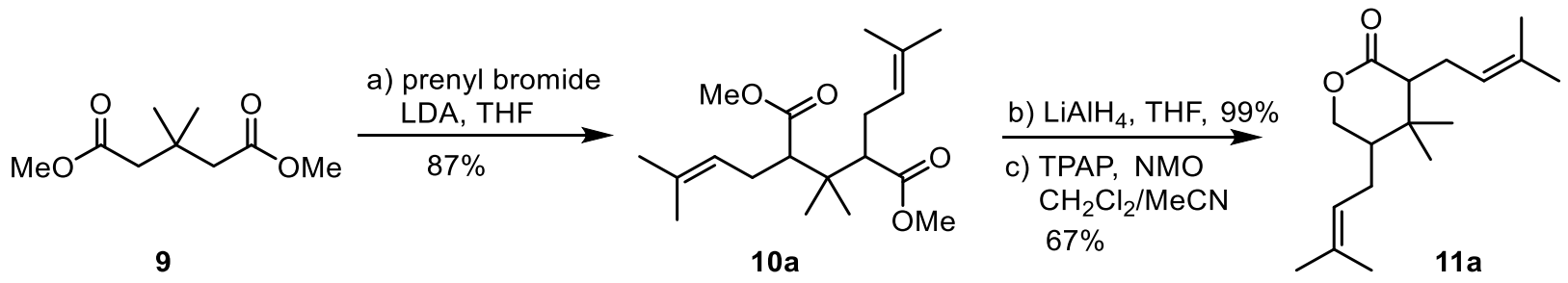

The compound 9 can be prepared according to the following procedure: A mixture of 3,3-dimethylpentanedioic acid $(45 \mathrm{~g}, 281 \mathrm{mmol})$ in methanol $(200 \mathrm{~mL})$ was added thionyl chloride $(45 \mathrm{~mL}, 618 \mathrm{mmol})$ dropwise. The solution was refluxed for 2 hours and concentrated under vacuum to afford compound 9 (52 g, $99 \%$ yield) which was used without further purification.

A $2000 \mathrm{~mL}$ of flame-dried flask charged with compound 9 (50 g, $265.9 \mathrm{mmol})$ and THF (500 mL) was cooled to $-78{ }^{\circ} \mathrm{C}$. A solution of lithium diisopropylamide ( $2 \mathrm{M}$ in THF, $273 \mathrm{~mL}, 546 \mathrm{mmol}$ ) was added dropwise. The solution was stirred for $30 \mathrm{~min}$ at $-78{ }^{\circ} \mathrm{C}$ and then 3, 3-dimethylallyl bromide $(95 \%, 66 \mathrm{~mL}, 546 \mathrm{mmol})$ in $\mathrm{THF}(10 \mathrm{~mL})$ was added dropwise. The reaction mixture was slowly warm to $0{ }^{\circ} \mathrm{C}$ and stirred at $0{ }^{\circ} \mathrm{C}$ for another 5 hours, and then quenched with saturated solution of $\mathrm{NH}_{4} \mathrm{Cl}(400 \mathrm{~mL})$. The aqueous phase was extracted with AcOEt $(3 \times 400 \mathrm{~mL})$, and the combined organic layers were dried over $\mathrm{Na}_{2} \mathrm{SO}_{4}$. Column chromatography yielded 10a (75 g, $87 \%$, cis : trans $\left.=2.5: 1\right)$ as pale yellow oils. $R_{f}=0.7$ (petroleum ether/ethyl acetate, 10:1). ${ }^{1} \mathrm{H}$ NMR $\left(400 \mathrm{MHz}, \mathrm{CDCl}_{3}\right) \delta 4.95-4.90(\mathrm{~m}, 2 \mathrm{H}), 3.61(\mathrm{~s}$, $6 \mathrm{H}), 2.44-2.08(\mathrm{~m}, 6 \mathrm{H}), 1.64(\mathrm{~s}, 6 \mathrm{H}),[1.58(\mathrm{~s}), 1.57(\mathrm{~s})](6 \mathrm{H}),[1.08(\mathrm{~s}), 1.00(\mathrm{~s}), 0.94(\mathrm{~s})](6 \mathrm{H}) .{ }^{13} \mathrm{C} \mathrm{NMR}(101 \mathrm{MHz}$, $\left.\mathrm{CDCl}_{3}\right) \delta 175.1,175.0,133.8,133.7,121.5,121.4,54.0,53.1,51.1,38.0,37.5,26.4,26.0,25.93,25.90,22.2,22.0,21.4$ 17.8, 17.7. IR (thin film) $v_{\max } 2971,2949,1732,1435,1359,1155 \mathrm{~cm}^{-1}$. HRESIMS calcd for $\mathrm{C}_{19} \mathrm{H}_{32} \mathrm{NaO}_{4}\left[\mathrm{M}+\mathrm{Na}^{+}\right.$ 347.2193, found 347.2200.

A solution of compounds 10a $(75 \mathrm{~g}, 231 \mathrm{mmol})$ in dry THF $(460 \mathrm{~mL})$ was cooled to $0{ }^{\circ} \mathrm{C}$. $\mathrm{LiAlH}_{4}(18 \mathrm{~g}, 473 \mathrm{mmol})$ powder was then carefully added in small portions during 15 min under $\mathrm{N}_{2}$. The resulting mixture was stirred for $2 \mathrm{~h}$ at room temperature, and then the reaction was slowly quenched with $18 \mathrm{~mL}$ of $\mathrm{H}_{2} \mathrm{O}, 18 \mathrm{~mL}$ of $15 \% \mathrm{NaOH}$ aq. and $54 \mathrm{~mL}$ of $\mathrm{H}_{2} \mathrm{O}$ at $0{ }^{\circ} \mathrm{C}$ in sequence. The suspension was stirred at room temperature for $15 \mathrm{~min}$, and anhydrous $\mathrm{MgSO}_{4}$ was added. After stirring for $15 \mathrm{~min}$, salts were filtered off through celite, and the residue was washed with AcOEt $(3 \times 30$ $\mathrm{mL}$ ). The combined organic layers were evaporated under reduced pressure to afford diols $\mathbf{S} 1$ as colorless oils in $99 \%$ yield $(61.3 \mathrm{~g}$, cis : trans $=2.3: 1)$ without further purification. $R_{f}=0.4$ (petroleum ether/ethyl acetate, $\left.3: 1\right)$. ${ }^{1} \mathrm{H}$ NMR $(400$ $\left.\mathrm{MHz}, \mathrm{CDCl}_{3}\right) \delta 5.25-5.22(\mathrm{~m}, 2 \mathrm{H}), 3.74(\mathrm{dd}, J=11.2,3.6 \mathrm{~Hz}, 2 \mathrm{H}), 3.59-3.53(\mathrm{~m}, 2 \mathrm{H}), 2.16-1.95(\mathrm{~m}, 4 \mathrm{H}), 1.70(\mathrm{~s}$, 
6H), 1.66-1.60 (m, 8H), [0.92 (s), $0.88(\mathrm{~s}), 0.86(\mathrm{~s})](6 \mathrm{H}) .{ }^{13} \mathrm{C}$ NMR $\left(151 \mathrm{MHz}, \mathrm{CDCl}_{3}\right) \delta 132.9,132.8,124.6,124.5,64.1$, 64.0, 48.4, 48.2, 38.1, 38.0, 26.9, 26.6, 26.0, 22.5, 22.2, 21.9, 18.0. IR (thin film) $v_{\max } 3388,2967,2916,1444,1376$, $1019 \mathrm{~cm}^{-1}$. HRESIMS calcd for $\mathrm{C}_{17} \mathrm{H}_{33} \mathrm{O}_{2}[\mathrm{M}+\mathrm{H}]^{+} 269.2475$, found 269.2486 .

To a solution of the above diols $\mathbf{S 1}(61.3 \mathrm{~g}, 229 \mathrm{mmol})$ in dry $\mathrm{CH}_{2} \mathrm{Cl}_{2}(460 \mathrm{~mL})$ and $\mathrm{MeCN}(46 \mathrm{~mL})(\mathrm{v} / \mathrm{v}=10: 1)$ was added powdered molecular sieves $(4 \AA, 61 \mathrm{~g})$ and 4-methylmorpholine $N$-oxide $(80.5 \mathrm{~g}, 687 \mathrm{mmol})$. tetra-Propylammonium perruthenate was then added in small portions $(4 \mathrm{~g}, 11.45 \mathrm{mmol})$ at $0{ }^{\circ} \mathrm{C}$. The mixture was stirred at $0{ }^{\circ} \mathrm{C}$ for $4 \mathrm{~h}$, filtered through silica and washed with AcOEt $(3 \times 50 \mathrm{~mL})$. The filtrate was concentrated under reduced pressure. Flash column chromatography yielded $11 \mathrm{a}(40.5 \mathrm{~g}, 67 \%$, cis : trans $=3: 1)$ as pale yellow oils. $R_{f}=0.6$ (petroleum ether/ethyl acetate, 10:1). ${ }^{1} \mathrm{H}$ NMR $\left(400 \mathrm{MHz}, \mathrm{CDCl}_{3}\right) \delta 5.29(\mathrm{t}, J=6.9 \mathrm{~Hz}, 0.5 \mathrm{H}), 5.21(\mathrm{t}, J=7.7 \mathrm{~Hz}, 0.5 \mathrm{H})$, $5.08-5.00(\mathrm{~m}, 1 \mathrm{H}), 4.27(\mathrm{dd}, J=11.8,5.5 \mathrm{~Hz}, 0.5 \mathrm{H}), 4.16(\mathrm{dd}, J=11.4,4.3 \mathrm{~Hz}, 0.5 \mathrm{H}), 4.01-3.89(\mathrm{~m}, 1 \mathrm{H}), 2.46-2.26$ $(\mathrm{m}, 1 \mathrm{H}), 2.25-2.07(\mathrm{~m}, 3 \mathrm{H}), 1.88-1.71(\mathrm{~m}, 2 \mathrm{H}),[1.69(\mathrm{~s}), 1.67(\mathrm{~s}), 1.63(\mathrm{~s}), 1.60(\mathrm{~s}), 1.59(\mathrm{~s})](12 \mathrm{H}),[1.11(\mathrm{~s}), 1.02$ (s), $0.96(\mathrm{~s}), 0.86(\mathrm{~s})](6 \mathrm{H}) .{ }^{13} \mathrm{C} \mathrm{NMR}\left(101 \mathrm{MHz}, \mathrm{CDCl}_{3}\right) \delta 174.8,174.2,134.1,132.7,131.7,124.3,122.9,121.8,121.7$, 69.7, 68.7, 53.6, 52.0, 45.04, 44.95, 36.6, 35.8, 26.9, 25.91, 25.88, 25.85, 25.7, 25.1, 24.9, 24.8, 24.4, 23.1, 18.0, 17.91, 17.88, 17.2. IR (thin film) $v_{\max } 2969,2917,1738,1453,1375,1147 \mathrm{~cm}^{-1}$. HRESIMS calcd for $\mathrm{C}_{17} \mathrm{H}_{29} \mathrm{O}_{2}[\mathrm{M}+\mathrm{H}]^{+}$ 265.2162, found 265.2163 .
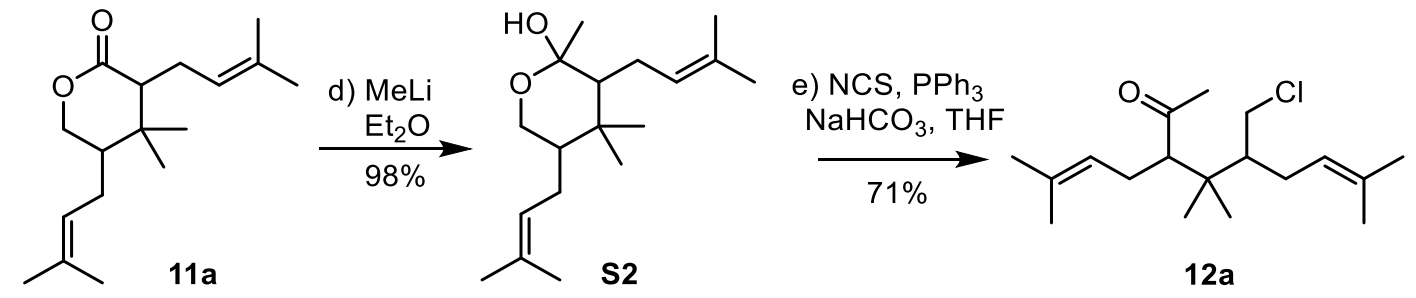

Compound 11a (40.5 g, $153.4 \mathrm{mmol})$ was dissolved in $150 \mathrm{~mL}$ of Et2O under $\mathrm{N}_{2}$. The solution was brought to $-78{ }^{\circ} \mathrm{C}$, and then a solution of methyl lithium $\left(1.6 \mathrm{M}_{\text {in }} \mathrm{Et}_{2} \mathrm{O}, 240 \mathrm{~mL}, 383.6 \mathrm{mmol}\right)$ was added dropwise over 40 minutes. The reaction was stirred at that temperature for 7 hours, and then $\mathrm{H}_{2} \mathrm{O}(40 \mathrm{~mL})$ was added dropwise. The product was extracted with AcOEt $(3 \times 30 \mathrm{~mL})$. The combined organic layers were dried over $\mathrm{Na}_{2} \mathrm{SO}_{4}$ and concentrated. The crude product $\mathbf{S 2}(42.1 \mathrm{~g}, 98 \%)$ was obtained as a pale yellow solid without further purification due to the instability. $R_{f}=0.6$ (petroleum ether/ethyl acetate, 10:1). A solution of $\mathbf{S 2}(42.1 \mathrm{~g}, 150.4 \mathrm{mmol})$ in dry THF $(600 \mathrm{~mL})$ at $-30{ }^{\circ} \mathrm{C}$ was stirred for $15 \mathrm{~min}$, sodium bicarbonate $(21.5 \mathrm{~g}, 255.6 \mathrm{mmol}), N$-chlorosuccinimide $(34.1 \mathrm{~g}, 255.6 \mathrm{mmol})$, and $\mathrm{PPh}_{3}(67 \mathrm{~g}, 255.6$ mmol) were successively added to the mixture. The mixture was then kept stirring at $-30{ }^{\circ} \mathrm{C}$ for 5 hours. The reaction was quenched by $30 \mathrm{~mL}$ of $\mathrm{H}_{2} \mathrm{O}$ and extracted with $\mathrm{CH}_{2} \mathrm{Cl}_{2}(3 \times 600 \mathrm{~mL})$. The combined organic extracts were washed with brine, dried over $\mathrm{Na}_{2} \mathrm{SO}_{4}$, filtered, concentrated, and purified by flash chromatography on silica gel to give 12a as yellow oils ( $15.9 \mathrm{~g}, 71 \% \mathrm{brsm}$, cis : trans $=2.6: 1) . R_{f}=0.8$ (petroleum ether/ethyl acetate, $\left.10: 1\right) .{ }^{1} \mathrm{H} \mathrm{NMR}(400 \mathrm{MHz}$, $\left.\mathrm{CDCl}_{3}\right) \delta 5.12-5.05(\mathrm{~m}, 1 \mathrm{H}), 4.99-4.90(\mathrm{~m}, 1 \mathrm{H}), 3.71-3.52(\mathrm{~m}, 2 \mathrm{H}), 2.85-2.75(\mathrm{~m}, 1 \mathrm{H}), 2.39-2.17(\mathrm{~m}, 3 \mathrm{H}),[2.12$ $(\mathrm{s}), 2.10(\mathrm{~s})](3 \mathrm{H}), 2.03(\mathrm{~m}, 1 \mathrm{H}),[1.71(\mathrm{~s}), 1.67(\mathrm{~s}), 1.65(\mathrm{~s}), 1.57(\mathrm{~s})](12 \mathrm{H}), 1.76-1.67(\mathrm{~m}, 1 \mathrm{H}),[1.06(\mathrm{~s}), 1.02(\mathrm{~s}), 1.00$ (s), 0.95 (s)] (6H). $\left.{ }^{13} \mathrm{C} \mathrm{NMR} \mathrm{(101} \mathrm{MHz,} \mathrm{CDCl}_{3}\right) \delta 213.7,213.5,133.8,133.7,133.6,133.1,123.2,123.0,121.86,121.85$, 58.4, 58.2, 47.43, 47.41, 46.1, 45.6, 39.33, 39.29, 35.0, 34.9, 26.9, 26.63, 26.57, 26.1, 25.91, 25.87, 22.6, 22.4, 22.3, 21.7, 18.1, 17.83, 17.79. IR (thin film) $v_{\max } 2969,2917,1710,1441,1353,1160 \mathrm{~cm}^{-1}$. HRESIMS calcd for $\mathrm{C}_{18} \mathrm{H}_{32} \mathrm{ClO}[\mathrm{M}+$ $\mathrm{H}]^{+}$299.2133, found 299.2147 . 


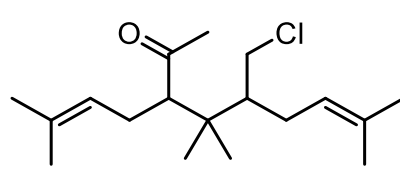

$12 \mathrm{a}$

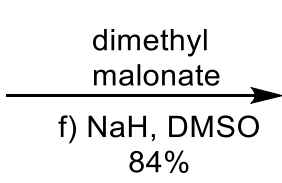

$84 \%$

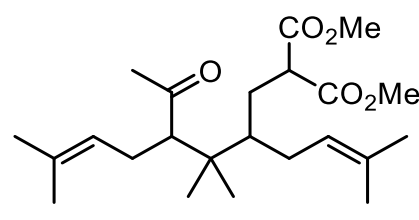

13a

A $2000 \mathrm{~mL}$ of round-bottomed flask was dried and charged with $\mathrm{NaH}(60 \%$ in mineral oil, $32.2 \mathrm{~g}, 804 \mathrm{mmol})$ and DMSO $(700 \mathrm{~mL})$ at $0{ }^{\circ} \mathrm{C}$. Dimethyl malonate $(96.5 \mathrm{~mL}, 844 \mathrm{~mol})$ in $100 \mathrm{~mL}$ of DMSO was added dropwise at $0{ }^{\circ} \mathrm{C}$. After $30 \mathrm{~min}$, compounds 12a (12 g, $40.2 \mathrm{mmol})$ in $30 \mathrm{~mL}$ of DMSO were added dropwise, following with sodium iodide $(121 \mathrm{~g}, 804 \mathrm{mmol})$. Then the reaction was stirred at $80^{\circ} \mathrm{C}$ for $24 \mathrm{~h}$. After cooling to room temperature, the reaction was quenched with $\mathrm{H}_{2} \mathrm{O}$ and extracted with AcOEt $(3 \times 800 \mathrm{~mL})$. The organic layers were dried over $\mathrm{Na}_{2} \mathrm{SO}_{4}$ and purified via flash chromatography to give products $13 \mathrm{a}$ as pale yellow oils $(13.3 \mathrm{~g}, 84 \%$, cis : trans $=3.6: 1)$. The cisand trans-isomers of products 13a were separated by pre-HPLC to confirm the structure. $R_{f}=0.25$ (petroleum ether/ethyl acetate, 10:1). cis-Isomer: ${ }^{1} \mathrm{H}$ NMR $\left(400 \mathrm{MHz}, \mathrm{CDCl}_{3}\right) \delta 5.05-4.96(\mathrm{~m}, 2 \mathrm{H}), 3.72(\mathrm{~s}, 6 \mathrm{H}), 3.54(\mathrm{dd}, J=11.0,4.0 \mathrm{~Hz}$, $1 \mathrm{H}), 2.80(\mathrm{dd}, J=11.6,2.8 \mathrm{~Hz}, 1 \mathrm{H}), 2.38-2.29(\mathrm{~m}, 1 \mathrm{H}), 2.23-2.17(\mathrm{~m}, 2 \mathrm{H}), 2.09(\mathrm{~s}, 3 \mathrm{H}), 1.98-1.85(\mathrm{~m}, 2 \mathrm{H}),[1.67$ $(\mathrm{s}), 1.64(\mathrm{~s}), 1.61(\mathrm{~s}), 1.57(\mathrm{~s})](12 \mathrm{H}), 1.66-1.58(\mathrm{~m}, 1 \mathrm{H}), 1.36-1.30(\mathrm{~m}, 1 \mathrm{H}), 0.94(\mathrm{~s}, 3 \mathrm{H}), 0.86(\mathrm{~s}, 3 \mathrm{H}) .{ }^{13} \mathrm{C} \mathrm{NMR}(101$ $\left.\mathrm{MHz}, \mathrm{CDCl}_{3}\right) \delta 213.7,170.4,170.0,133.4,132.2,124.3,122.2,57.5,52.7,52.5,50.7,42.8,34.7,30.6,28.9,26.8,26.0$, 25.9, 21.6, 21.5, 18.1, 17.8. IR (thin film) $v_{\max } 2968,2924,1738,1709,1435,1152 \mathrm{~cm}^{-1}$. HRESIMS calcd for $\mathrm{C}_{23} \mathrm{H}_{39} \mathrm{O}_{5}$ $[\mathrm{M}+\mathrm{H}]^{+}$395.2792, found 395.2798. trans-Isomer: ${ }^{1} \mathrm{H} \mathrm{NMR}\left(600 \mathrm{MHz}, \mathrm{CDCl}_{3}\right) \delta 5.05(\mathrm{t}, J=7.1 \mathrm{~Hz}, 1 \mathrm{H}), 4.90(\mathrm{t}, J=$ $7.3 \mathrm{~Hz}, 1 \mathrm{H}), 3.73(\mathrm{~s}, 3 \mathrm{H}), 3.72(\mathrm{~s}, 3 \mathrm{H}), 3.51(\mathrm{dd}, J=10.7,4.4 \mathrm{~Hz}, 1 \mathrm{H}), 2.77(\mathrm{dd}, J=11.4,2.6 \mathrm{~Hz}, 1 \mathrm{H}), 2.34-2.28(\mathrm{~m}$, $1 \mathrm{H}), 2.22-2.19(\mathrm{~m}, 1 \mathrm{H}), 2.17-2.12(\mathrm{~m}, 1 \mathrm{H}), 2.10(\mathrm{~s}, 3 \mathrm{H}), 2.00-1.96(\mathrm{~m}, 1 \mathrm{H}), 1.94-1.89(\mathrm{~m}, 1 \mathrm{H}), 1.69(\mathrm{~s}, 3 \mathrm{H}), 1.65$ $(\mathrm{s}, 3 \mathrm{H}), 1.64(\mathrm{~s}, 3 \mathrm{H}), 1.67-1.60(\mathrm{~m}, 1 \mathrm{H}), 1.57(\mathrm{~s}, 3 \mathrm{H}), 1.42-1.38(\mathrm{~m}, 1 \mathrm{H}), 0.97(\mathrm{~s}, 3 \mathrm{H}), 0.85(\mathrm{~s}, 3 \mathrm{H}) .{ }^{13} \mathrm{C} \mathrm{NMR}(151$ $\left.\mathrm{MHz}, \mathrm{CDCl}_{3}\right) \delta 213.6,170.4,170.0,133.4,132.1,124.2,122.1,57.5,52.7,52.5,51.1,42.9,39.3,35.0,30.0,29.4,26.5$, 26.02, 25.95, 21.6, 21.4, 18.1, 17.8. IR (thin film) $v_{\max } 2969,2916,1732,1435,1219,1081 \mathrm{~cm}^{-1}$. HRESIMS calcd for $\mathrm{C}_{23} \mathrm{H}_{39} \mathrm{O}_{5}[\mathrm{M}+\mathrm{H}]^{+}$395.2792, found 395.2805.

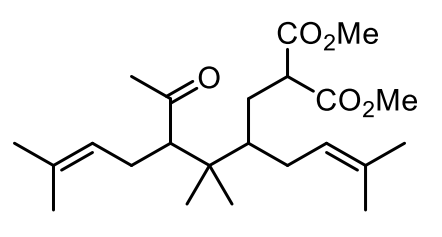

$13 a$

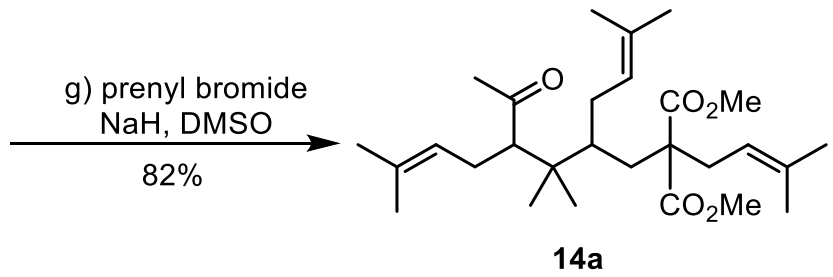

Compounds 13a (7.6 g, $19.3 \mathrm{mmol})$ were added dropwise to a stirred suspension of $\mathrm{NaH}(60 \%$ in mineral oil, $1.2 \mathrm{~g}$, $29 \mathrm{mmol})$ in DMSO $(150 \mathrm{ml})$ at $0{ }^{\circ} \mathrm{C}$. The resulting mixture was stirred at $50{ }^{\circ} \mathrm{C}$ for $0.5 \mathrm{~h}$. After the reaction was cooled down to room temperature, the prenyl bromide $(95 \%, 3.5 \mathrm{~mL}, 29 \mathrm{mmol})$ was added and the mixture was stirred for 6 hours. The reaction was quenched with $\mathrm{H}_{2} \mathrm{O}$ and extracted with AcOEt $(3 \times 150 \mathrm{~mL})$. The combined organic layers were washed with brine, dried over $\mathrm{Na}_{2} \mathrm{SO}_{4}$, filtered, and the solvents were evaporated under reduced pressure to give the crude product, which was purified by flash chromatography. Compounds 14a were obtained as pale yellow oils (7.3 g, $82 \%$, cis : trans $=3: 1) . R_{f}=0.5$ (petroleum ether/ethyl acetate, 10:1). ${ }^{1} \mathrm{H}$ NMR $\left(400 \mathrm{MHz}, \mathrm{CDCl}_{3}\right) \delta 5.10-4.74(\mathrm{~m}$, $3 \mathrm{H}), 3.65(\mathrm{~s}, 3 \mathrm{H}), 3.63(\mathrm{~s}, 3 \mathrm{H}), 2.75-2.51(\mathrm{~m}, 3 \mathrm{H}), 2.37-2.15(\mathrm{~m}, 2 \mathrm{H}), 2.05-1.93(\mathrm{~m}, 4 \mathrm{H}), 1.87-1.74(\mathrm{~m}, 3 \mathrm{H}), 1.66$ -1.47 (m, 19H), [0.87 (s), 0.85 (s), $0.82(\mathrm{~s}), 0.80(\mathrm{~s})](6 \mathrm{H}) .{ }^{13} \mathrm{C} \mathrm{NMR}\left(101 \mathrm{MHz}, \mathrm{CDCl}_{3}\right) \delta 213.9,213.4,172.52,172.45$, 172.4 , 135.63, 135.56, 133.2, 132.9, 130.1, 130.0, 125.2, 125.1, 122.3, 122.1, 117.8, 57.5, 57.3, 56.9, 56.4, 52.44, 52.36, 52.3, 40.06, 40.05, 39.91, 39.86, 35.3, 34.6, 34.0, 33.4, 31.8, 31.4, 30.0, 28.8, 27.2, 26.3, 26.1, 26.0, 25.92, 25.88, 25.8, 22.1, 21.5, 21.1, 18.2, 18.1, 18.0, 17.81, 17.78. HRESIMS calcd for $\mathrm{C}_{28} \mathrm{H}_{47} \mathrm{O}_{5}[\mathrm{M}+\mathrm{H}]^{+} 463.3418$, found 463.3426 . 
<smiles>CC(=O)CC(CC=C(C)C)C(CC=C(C)C)C(C)=O</smiles>

$13 a$

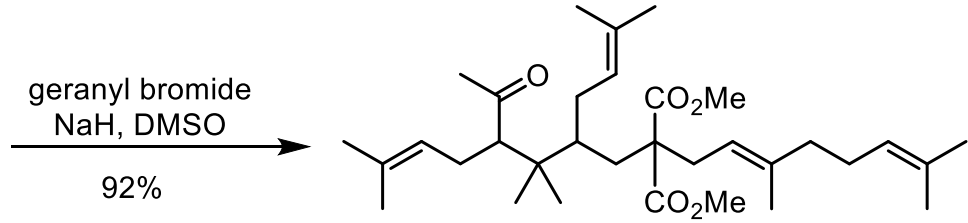

14b

Compounds 14b were prepared according to the general procedure that used for 14a with 13a (4.8 $\mathrm{g}, 12.1 \mathrm{mmol})$, $\mathrm{NaH}(60 \%$ in mineral oil, $728 \mathrm{mg}, 18.2 \mathrm{mmol})$ and geranyl bromide $(96 \%, 3.8 \mathrm{~mL}, 18.2 \mathrm{mmol})$. The residue was purified by flash column chromatography to yield the desired product $\mathbf{1 4 b}(5.9 \mathrm{~g}, 92 \%$, cis : trans $=3.2: 1)$ as pale yellow oils. $R_{f}=0.55$ (petroleum ether/ethyl acetate, 10:1). ${ }^{1} \mathrm{H}$ NMR $\left(400 \mathrm{MHz}, \mathrm{CDCl}_{3}\right) \delta 5.10-4.64(\mathrm{~m}, 4 \mathrm{H}), 3.66-$ $3.65(\mathrm{~m}, 6 \mathrm{H}), 2.76-2.53(\mathrm{~m}, 3 \mathrm{H}), 2.37-2.17(\mathrm{~m}, 2 \mathrm{H}), 2.07-1.75(\mathrm{~m}, 11 \mathrm{H}), 1.67-1.49(\mathrm{~m}, 22 \mathrm{H}),[0.90(\mathrm{~s}), 0.86(\mathrm{~s})$, $0.84(\mathrm{~s}), 0.82(\mathrm{~s})](6 \mathrm{H}) .{ }^{13} \mathrm{C} \mathrm{NMR}\left(101 \mathrm{MHz}, \mathrm{CDCl}_{3}\right) \delta 213.9,213.4,172.5,172.51,172.46,172.4,139.4,139.3,133.2$, 133.0, 131.6, 130.1, 125.3, 125.2, 124.13, 124.11, 122.4, 122.1, 117.74, 117.70, 57.5, 57.4, 57.0, 56.4, 52.44, 52.38, 52.3, 40.2 , 40.11, 40.09, 40.02, 39.97, 39.9, 35.4, 34.6, 34.2, 33.4, 31.8, 31.3, 30.1, 29.0, 27.3, 26.8, 26.7, 26.4, 26.01, 25.95, $25.91,25.87,25.8,22.1,21.53,21.50,21.2,18.2,18.1,17.9,17.83,17.82,16.5,16.4$. IR (thin film) $v_{\max } 2968,2917$, $1737,1711,1435,1216 \mathrm{~cm}^{-1}$. HRESIMS calcd for $\mathrm{C}_{33} \mathrm{H}_{55} \mathrm{O}_{5}[\mathrm{M}+\mathrm{H}]^{+} 531.4129$, found 531.4056 .

\section{Scalable synthesis of the functionalized linear precursors $14 \mathrm{c}, 14 \mathrm{~d}, 14 \mathrm{e}, 14 \mathrm{f}$}<smiles>COC(=O)CC(C)(C)CC(=O)OC</smiles>

9 a) prenyl bromide

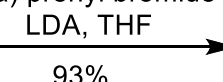

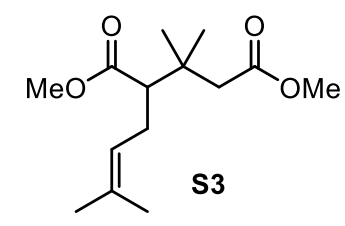

b) geranyl bromide LDA, THF

$96 \%$

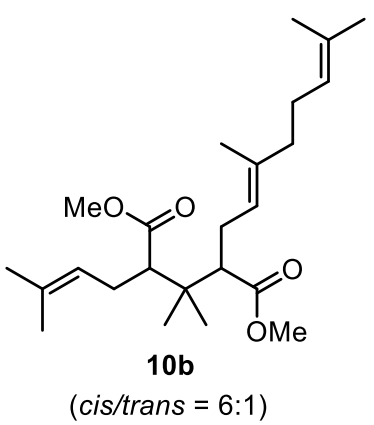

Compound $\mathbf{S 3}$ was prepared according to the general procedure that used for 10a with compound $\mathbf{9}$ (23 $\mathrm{g}, 122 \mathrm{mmol})$, lithium diisopropylamide (2 M in THF, $64 \mathrm{~mL}, 128 \mathrm{mmol})$ and prenyl bromide $(95 \%, 15.6 \mathrm{~mL}, 128 \mathrm{mmol})$. The residue was purified by column chromatography over silica gel to yield the desired product $\mathbf{S 3}(28.9 \mathrm{~g}, 93 \%)$ as a pale yellow oil. $R_{f}=0.65$ (petroleum ether/ethyl acetate, 10:1). ${ }^{1} \mathrm{H}$ NMR $\left(400 \mathrm{MHz}, \mathrm{CDCl}_{3}\right) \delta 4.96(\mathrm{t}, J=7.3 \mathrm{~Hz}, 1 \mathrm{H}), 3.64(\mathrm{~s}, 3 \mathrm{H}), 3.61$ $(\mathrm{s}, 3 \mathrm{H}), 2.41-2.26(\mathrm{~m}, 4 \mathrm{H}), 2.14(\mathrm{dd}, J=12.7,6.5 \mathrm{~Hz}, 1 \mathrm{H}), 1.64(\mathrm{~s}, 3 \mathrm{H}), 1.58(\mathrm{~s}, 3 \mathrm{H}), 1.07(\mathrm{~s}, 3 \mathrm{H}), 1.06(\mathrm{~s}, 3 \mathrm{H}) .{ }^{13} \mathrm{C}$ NMR $\left(101 \mathrm{MHz} \mathrm{CDCl}_{3}\right) \delta 175.0,172.4,133.8,121.4,55.2,51.4,51.2,44.1,35.3,26.2,25.9,25.5,25.1$, 17.7. IR (thin film) $v_{\max } 2952,1737,1435,1206,1155 \mathrm{~cm}^{-1}$. HRESIMS calcd for $\mathrm{C}_{14} \mathrm{H}_{24} \mathrm{NaO}_{4}[\mathrm{M}+\mathrm{Na}]^{+} 279.1567$, found 279.1566 .

A $500 \mathrm{~mL}$ of flame-dried flask at $-78{ }^{\circ} \mathrm{C}$ was charged with compound $\mathbf{S 3}$ (28 g, $\left.109.8 \mathrm{mmol}\right)$ and THF (100 mL). A solution of lithium diisopropylamide ( $2 \mathrm{M}$ in THF, $57.4 \mathrm{~mL}, 115 \mathrm{mmol}$ ) was added dropwise. The solution was stirred for $30 \mathrm{~min}$ at $-78{ }^{\circ} \mathrm{C}$ and then geranyl bromide $(96 \%, 23.8 \mathrm{~mL}, 115 \mathrm{mmol})$ in THF $(20 \mathrm{~mL})$ was added dropwise. The reaction mixture was slowly warmed to $0{ }^{\circ} \mathrm{C}$ and stirred at $0{ }^{\circ} \mathrm{C}$ for another 5 hours, and then quenched with saturated solution of $\mathrm{NH}_{4} \mathrm{Cl}(100 \mathrm{~mL})$. The aqueous phase was extracted with AcOEt $(3 \times 200 \mathrm{~mL})$, and the combined organic layers were dried over $\mathrm{Na}_{2} \mathrm{SO}_{4}$. Column chromatography yielded esters $\mathbf{1 0 b}(41 \mathrm{~g}, 96 \%$, cis/trans $=6: 1)$ as yellow oils. $R_{f}=0.75$ (petroleum ether/ethyl acetate, 10:1). ${ }^{1} \mathrm{H}$ NMR (400 MHz, $\left.\mathrm{CDCl}_{3}\right) \delta 5.05(\mathrm{~m}, 1 \mathrm{H}), 4.94(\mathrm{~m}, 2 \mathrm{H}), 3.62(\mathrm{~s}, 3 \mathrm{H})$, $3.61(\mathrm{~s}, 3 \mathrm{H}), 2.46-2.04(\mathrm{~m}, 6 \mathrm{H}), 2.03-1.92(\mathrm{~m}, 4 \mathrm{H})$, [1.66 (s), $1.65(\mathrm{~s}), 1.59(\mathrm{~s}), 1.58(\mathrm{~s})](15 \mathrm{H})$, [1.10 (s), $1.02(\mathrm{~s})$, $0.96(\mathrm{~s})](6 \mathrm{H}) .{ }^{13} \mathrm{C} \mathrm{NMR}\left(101 \mathrm{MHz}, \mathrm{CDCl}_{3}\right) \delta 175.0,137.4,133.7,131.5,124.3,121.54,121.52,54.1,54.0,51.2,51.1$, 
39.9, 38.0, 26.8, 26.1, 25.99, 25.95, 25.8, 22.3, 21.5, 17.80, 17.77, 16.1. IR (thin film) $v_{\max } 2969,2927,1737,1435,1199$, $1156 \mathrm{~cm}^{-1}$. HRESIMS calcd for $\mathrm{C}_{24} \mathrm{H}_{40} \mathrm{NaO}_{4}[\mathrm{M}+\mathrm{Na}]^{+} 415.2819$, found 415.2824 .

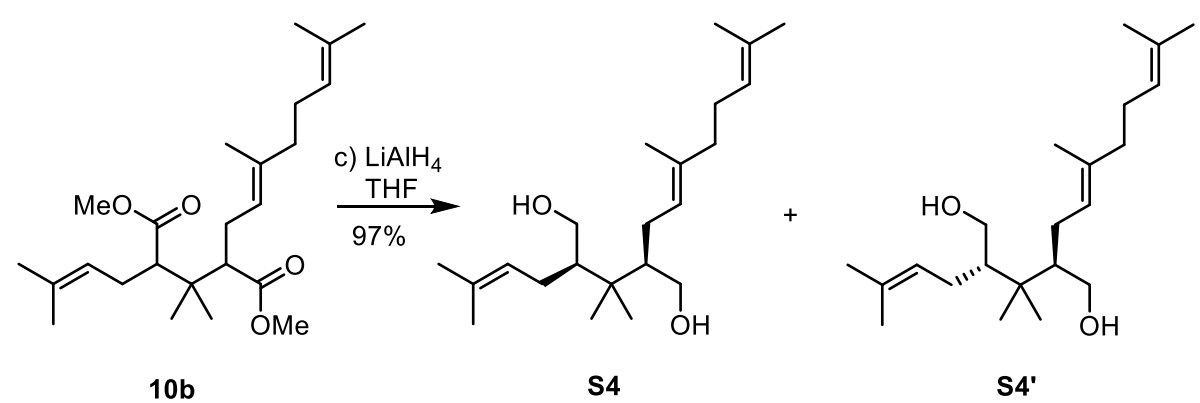

Compounds S4/S4' were prepared according to the general procedure that used for $\mathbf{S 1}$ with compound $\mathbf{1 0 b}$ (39.2 g, $100 \mathrm{mmol})$ and $\mathrm{LiAlH}_{4}(8.2 \mathrm{~g}, 205 \mathrm{mmol})$ in THF. The residue was purified by column chromatography over silica gel to

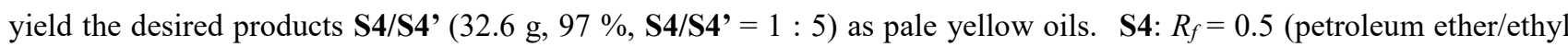
acetate, 3:1). ${ }^{1} \mathrm{H}$ NMR $\left(400 \mathrm{MHz}, \mathrm{CDCl}_{3}\right) \delta 5.22-5.14(\mathrm{~m}, 2 \mathrm{H}), 5.00(\mathrm{t}, J=6.7 \mathrm{~Hz}, 1 \mathrm{H}), 3.70(\mathrm{t}, J=3.2 \mathrm{~Hz}, 1 \mathrm{H}), 3.67(\mathrm{t}$, $J=3.2 \mathrm{~Hz}, 1 \mathrm{H}), 3.53(\mathrm{dd}, J=5.7,2.6 \mathrm{~Hz}, 1 \mathrm{H}), 3.50(\mathrm{dd}, J=5.7,2.7 \mathrm{~Hz}, 1 \mathrm{H}), 2.11-1.88(\mathrm{~m}, 8 \mathrm{H}), 1.64(\mathrm{~s}, 3 \mathrm{H}), 1.64-$ $1.62(\mathrm{~m}, 2 \mathrm{H}), 1.61(\mathrm{~s}, 3 \mathrm{H}), 1.59(\mathrm{~s}, 6 \mathrm{H}), 1.58-1.55(\mathrm{~m}, 2 \mathrm{H}), 1.53(\mathrm{~s}, 3 \mathrm{H}), 0.87(\mathrm{~s}, 3 \mathrm{H}), 0.80(\mathrm{~s}, 3 \mathrm{H}) .{ }^{13} \mathrm{C}$ NMR $(101 \mathrm{MHz}$, $\left.\mathrm{CDCl}_{3}\right) \delta 136.5,132.9,131.9,124.64,124.60,124.2,64.12,64.10,48.23,48.19,40.0,38.0,26.7,26.6,26.0,25.8,22.6$, 22.2, 18.0, 17.8, 16.3. IR (thin film) $v_{\max } 3399,2918,1446,1376,1192,1019 \mathrm{~cm}^{-1}$. HRESIMS calcd for $\mathrm{C}_{22} \mathrm{H}_{41} \mathrm{O}_{2}[\mathrm{M}+$ $\mathrm{H}]^{+}$337.3101, found 337.3108. S4': $R_{f}=0.55$ (petroleum ether/ethyl acetate, $\left.3: 1\right) .{ }^{1} \mathrm{H}$ NMR (400 MHz, $\left.\mathrm{CDCl}_{3}\right) \delta 5.27-$ $5.18(\mathrm{~m}, 2 \mathrm{H}), 5.06(\mathrm{t}, J=6.8 \mathrm{~Hz}, 1 \mathrm{H}), 3.75(\mathrm{dd}, J=4.4,2.3 \mathrm{~Hz}, 1 \mathrm{H}), 3.73(\mathrm{dd}, J=4.4,2.3 \mathrm{~Hz}, 1 \mathrm{H}), 3.56(\mathrm{~d}, J=5.0 \mathrm{~Hz}$, $1 \mathrm{H}), 3.54(\mathrm{~d}, J=5.0 \mathrm{~Hz}, 1 \mathrm{H}), 2.14-1.91(\mathrm{~m}, 10 \mathrm{H}),[1.70(\mathrm{~s}), 1.67(\mathrm{~s}), 1.64(\mathrm{~s}), 1.59(\mathrm{~s})](15 \mathrm{H}), 1.69-1.65(\mathrm{~m}, 2 \mathrm{H}), 0.89$ (s, 6H). ${ }^{13} \mathrm{C}$ NMR (101 MHz, $\left.\mathrm{CDCl}_{3}\right) \delta 136.4,132.8,131.8,124.53,124.47,124.2,64.0,48.5,40.0,38.1,26.9,26.8,26.7$, 26.0, 25.8, 22.0, 18.0, 17.8, 16.3. IR (thin film) $v_{\max } 3407,2964,2925,1742,1453,1376,1245,1190,1082,1029,847$ $\mathrm{cm}^{-1}$. HRESIMS calcd for $\mathrm{C}_{22} \mathrm{H}_{41} \mathrm{O}_{2}[\mathrm{M}+\mathrm{H}]^{+} 337.3101$, found 337.3110 .
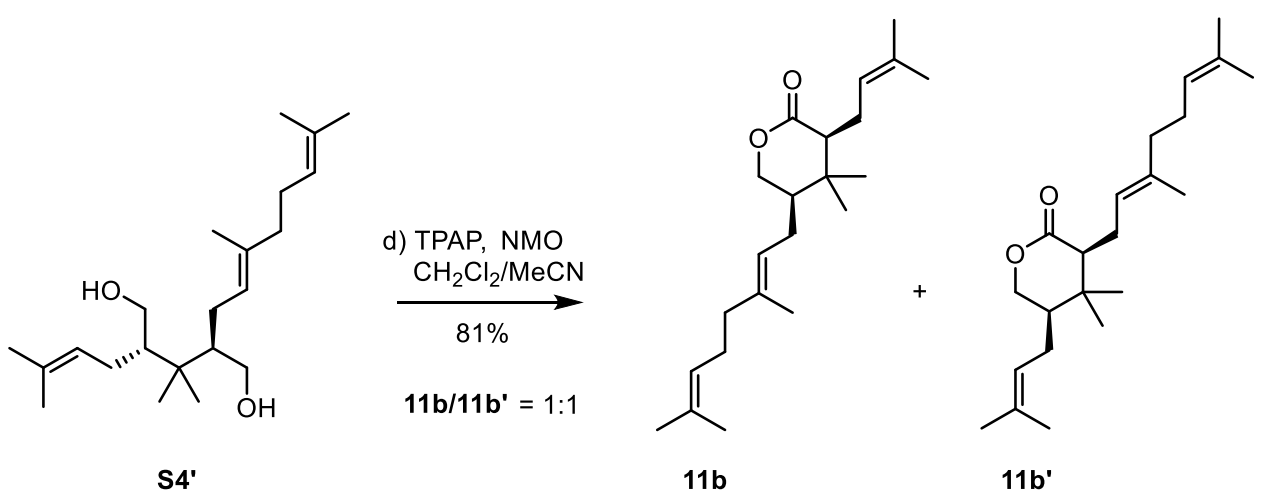

Compounds 11b/11b' were prepared according to the general procedure that used for 11a with compounds S4' (21 g, $62.5 \mathrm{mmol})$, molecular sieves (4 A, $21 \mathrm{~g})$, 4-methylmorpholine $N$-oxide (22 g, $187.5 \mathrm{mmol})$ and tetra-propylammonium perruthenate $(1.1 \mathrm{~g}, 3.125 \mathrm{mmol})$. The residue was purified by column chromatography over silica gel to yield the desired products $\mathbf{1 1} \mathbf{b} / \mathbf{1 1} \mathbf{b}^{\prime}\left(16.8 \mathrm{~g}, 81 \%, \mathbf{1 1} \mathbf{b} / \mathbf{1 1} \mathbf{b}^{\prime}=1: 1\right)$ as pale yellow oils. $R_{f}=0.65$ (petroleum ether/ethyl acetate, $\left.10: 1\right) .{ }^{1} \mathrm{H}$ NMR (400 MHz, $\left.\mathrm{CDCl}_{3}\right) \delta 5.29(\mathrm{~m}, 1 \mathrm{H}), 5.08-5.00(\mathrm{~m}, 2 \mathrm{H}), 4.29-4.24(\mathrm{~m}, 1 \mathrm{H}), 3.99-3.93(\mathrm{~m}, 1 \mathrm{H}), 2.48-2.37(\mathrm{~m}$, $1 \mathrm{H}), 2.28-2.13(\mathrm{~m}, 3 \mathrm{H}), 2.05-1.95(\mathrm{~m}, 4 \mathrm{H}), 1.87-1.72(\mathrm{~m}, 2 \mathrm{H}),[1.69(\mathrm{~s}), 1.67(\mathrm{~s}), 1.65(\mathrm{~s})](6 \mathrm{H}), 1.63(\mathrm{~s}, 3 \mathrm{H}),[1.59$ (s), $1.58(\mathrm{~s})](6 \mathrm{H}), 1.11(\mathrm{~s}, 3 \mathrm{H}), 0.86(\mathrm{~s}, 3 \mathrm{H}) .{ }^{13} \mathrm{C} \mathrm{NMR}\left(101 \mathrm{MHz}, \mathrm{CDCl}_{3}\right) \delta 174.2,174.1,137.5,135.2,134.0,131.71$, 131.66, 131.4, 124.4, 124.3, 124.1, 121.69, 121.65, 69.7, 69.6, 53.6, 53.5, 45.0, 44.9, 39.8, 36.6, 26.9, 26.7, 26.5, 25.9, 
$25.8,25.64,25.56,24.33,24.27,17.92,17.88,17.8,17.2,16.2$. IR (thin film) $v_{\max } 2969,2916,1739,1450,1376,1148$ $\mathrm{cm}^{-1}$. HRESIMS calcd for $\mathrm{C}_{22} \mathrm{H}_{37} \mathrm{O}_{2}[\mathrm{M}+\mathrm{H}]^{+} 333.2788$, found 333.2804.

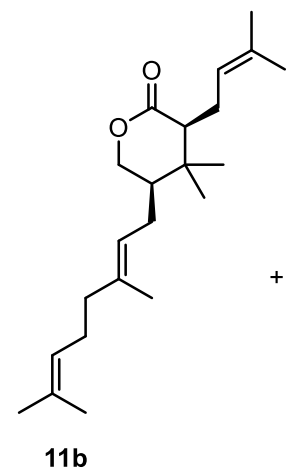

$11 \mathrm{~b}$

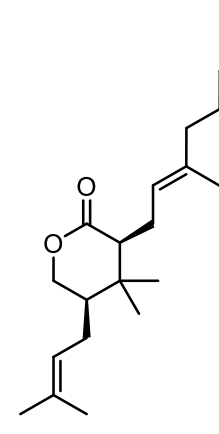

$11 b^{\prime}$<smiles>CC(=O)[C@H](CC=C(C)C)C(C)(C)[C@H](CCl)C/C=C(\C)CCC=C(C)C</smiles>

$12 b$

e) $\mathrm{MeLi}, \mathrm{Et}_{2} \mathrm{O}$

f) $\mathrm{NCS}, \mathrm{PPh}_{3}$ $\underset{59 \%}{\mathrm{NaHCO}_{3}, \mathrm{THF}}$<smiles>CC(=O)[C@H](C/C=C(\C)CCC=C(C)C)C(C)(C)[C@H](CCl)CC=C(C)C</smiles>

$12 b^{\prime}$

According to similar procedures that used for the synthesis of $\mathbf{1 2 a}$, compounds $\mathbf{1 2 b} / \mathbf{1 2 b} \mathbf{b}^{\prime}(16.8 \mathrm{~g})$ were prepared from 11b/11b' $\left(10.9 \mathrm{~g}, \mathbf{1 2 b} / \mathbf{1 2} \mathbf{b}^{\prime}=1.2: 1\right)$ in $59 \%$ yield as a mixture. Pale yellow oils. $R_{f}=0.8$ (petroleum ether/ethyl acetate, 10:1). ${ }^{1} \mathrm{H}$ NMR $\left(400 \mathrm{MHz}, \mathrm{CDCl}_{3}\right) \delta 5.08-4.95(\mathrm{~m}, 3 \mathrm{H}), 3.65(\mathrm{dd}, J=11.6,3.7 \mathrm{~Hz}, 1 \mathrm{H}), 3.56(\mathrm{dt}, J=11.5,3.0,1 \mathrm{H})$, $2.83(\mathrm{dd}, J=11.5,2.6 \mathrm{~Hz}, 1 \mathrm{H}), 2.12(\mathrm{~s}, 3 \mathrm{H}), 2.40-1.93(\mathrm{~m}, 9 \mathrm{H}), 1.70-1.59(\mathrm{~m}, 15 \mathrm{H}), 1.02(\mathrm{~s}, 3 \mathrm{H}), 1.00(\mathrm{~s}, 3 \mathrm{H}) .{ }^{13} \mathrm{C}$ NMR $\left(101 \mathrm{MHz}, \mathrm{CDCl}_{3}\right) \delta 213.71,213.65,137.41,137.36,133.8,133.7,131.7,124.4,124.3,123.1,123.1,122.0,121.9$, 67.6, 58.3, 47.54, 47.50, 45.7, 45.6, 40.1, 40.0, 39.4, 35.2, 35.1, 26.8, 26.74, 26.72, 26.66, 26.1, 25.99, 25.96, 25.9, 25.8, 22.5, 22.4, 18.2, 17.9, 16.4, 16.2. IR (thin film) $v_{\max } 2967,2918,1711,1439,1375,1159 \mathrm{~cm}^{-1}$. HRESIMS calcd for $\mathrm{C}_{23} \mathrm{H}_{40} \mathrm{ClO}[\mathrm{M}+\mathrm{H}]^{+} 367.2762$, found 367.2765 .

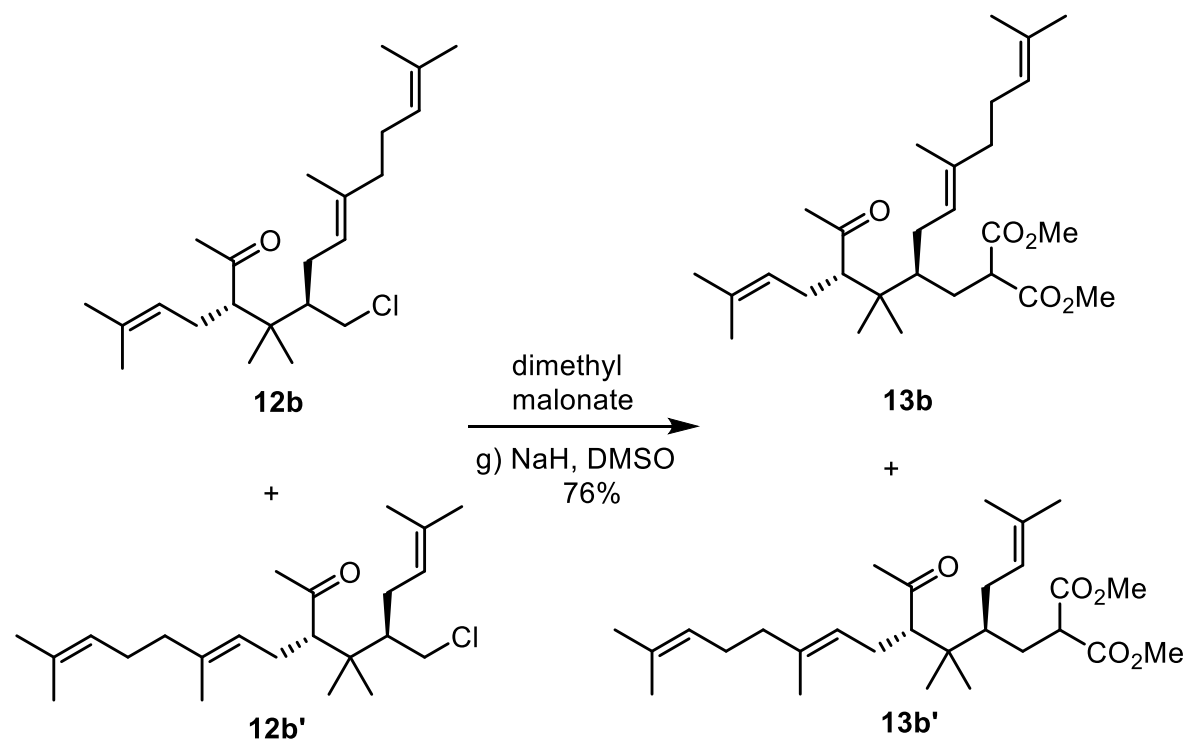

Compounds 13b/13b' were prepared according to the general procedure that used for 13a with 12b/12b' (10.8 g, 29.5 $\mathrm{mmol}), \mathrm{NaH}$ (60\% in mineral oil, $23.6 \mathrm{~g}, 590 \mathrm{mmol})$, sodium iodide $(37.5 \mathrm{~g}, 250 \mathrm{mmol})$ and dimethyl malonate (70.8 $\mathrm{mL}$, $620 \mathrm{mmol}$ ). The residue was purified by column chromatography over silica gel to yield the desired products $\mathbf{1 3 b} / \mathbf{1 3 b}$ ' $\left(10.3 \mathrm{~g}, 76 \%, \mathbf{1 3 b} / \mathbf{1 3 b} \mathbf{b}^{\prime}=1.5: 1\right)$ as pale yellow oils. The regio-isomers of products $\mathbf{1 3 b} / \mathbf{1 3 b}$ ' were separated by prep-HPLC to confirm the structure. 13b' (contaminated with 13b): $R_{f}=0.34$ (petroleum ether/ethyl acetate, $10: 1$ ). ${ }^{1} \mathrm{H}$ NMR (400 MHz, $\left.\mathrm{CDCl}_{3}\right) \delta 5.09-4.96(\mathrm{~m}, 3 \mathrm{H}), 3.714(\mathrm{~s}, 3 \mathrm{H}), 3.709(\mathrm{~s}, 3 \mathrm{H}), 3.57$ (dd, $\left.J=11.2,3.8 \mathrm{~Hz}, 1 \mathrm{H}\right), 2.81(\mathrm{dd}, J$ 
$=11.6,2.8 \mathrm{~Hz}, 1 \mathrm{H}), 2.39-2.30(\mathrm{~m}, 1 \mathrm{H}), 2.24-2.18(\mathrm{~m}, 2 \mathrm{H}), 2.09(\mathrm{~s}, 3 \mathrm{H}), 2.06-2.03(\mathrm{~m}, 2 \mathrm{H}), 1.99-1.93(\mathrm{~m}, 4 \mathrm{H})$ $1.91-1.87(\mathrm{~m}, 1 \mathrm{H}),[1.66(\mathrm{~s}), 1.64(\mathrm{~s}), 1.61(\mathrm{~s}), 1.59(\mathrm{~s}), 1.56(\mathrm{~s})](15 \mathrm{H}), 1.37-1.31(\mathrm{~m}, 1 \mathrm{H}), 0.94(\mathrm{~s}, 3 \mathrm{H}), 0.86(\mathrm{~s}, 3 \mathrm{H})$. ${ }^{13} \mathrm{C}$ NMR $\left(151 \mathrm{MHz}, \mathrm{CDCl}_{3}\right) \delta 213.7,170.4,170.1,135.8,133.4,131.6,124.4,124.1,122.2,57.4,52.7,52.5,50.7,42.7$, 40.0, 39.4, 34.8, 30.7, 28.7, 26.8, 26.6, 26.0, 25.8, 21.7, 21.5, 17.83, 17.79, 16.4. IR (thin film) $v_{\max } 2967,2925,1738$, $1709,1435,1352,1200,1152 \mathrm{~cm}^{-1}$. HRESIMS calcd for $\mathrm{C}_{28} \mathrm{H}_{47} \mathrm{O}_{5}[\mathrm{M}+\mathrm{H}]^{+} 463.3418$, found 463.3419. 13b: $R_{f}=0.35$ (petroleum ether/ethyl acetate, 10:1). ${ }^{1} \mathrm{H}$ NMR $\left(400 \mathrm{MHz}, \mathrm{CDCl}_{3}\right) \delta 5.06-5.00(\mathrm{~m}, 3 \mathrm{H}), 3.72(\mathrm{~s}, 6 \mathrm{H}), 3.55(\mathrm{dd}, J=11.0$, $4.1 \mathrm{~Hz}, 1 \mathrm{H}), 2.81(\mathrm{dd}, J=11.6,2.8 \mathrm{~Hz}, 1 \mathrm{H}), 2.39-2.33(\mathrm{~m}, 1 \mathrm{H}), 2.24-2.18(\mathrm{~m}, 2 \mathrm{H}), 2.09(\mathrm{~s}, 3 \mathrm{H}), 2.05-2.01(\mathrm{~m}, 2 \mathrm{H})$, $1.99-1.94(\mathrm{~m}, 4 \mathrm{H}), 1.91-1.87(\mathrm{~m}, 1 \mathrm{H}),[1.68(\mathrm{~s}), 1.66(\mathrm{~s}), 1.62(\mathrm{~s}), 1.58(\mathrm{~s}), 1.57(\mathrm{~s})](15 \mathrm{H}), 1.36-1.34(\mathrm{~m}, 1 \mathrm{H}), 0.95$ (s, 3H), 0.87 (s, 3H). ${ }^{13} \mathrm{C}$ NMR (151 MHz, $\left.\mathrm{CDCl}_{3}\right) \delta 213.6,170.4,170.0,137.1,132.2,131.6,124.34,124.30,122.2$, $57.5,52.7,52.5,50.8,42.8,40.0,39.5,34.9,30.7,28.9,26.8,26.7,26.0,25.8,21.6,21.5,18.1,17.8,16.1$. IR (thin film) $v_{\max } 2964,2925,1738,1710,1435,1352,1191,1152,967 \mathrm{~cm}^{-1}$. HRESIMS calcd for $\mathrm{C}_{28} \mathrm{H}_{47} \mathrm{O}_{5}[\mathrm{M}+\mathrm{H}]^{+} 463.3418$, found 463.3434 .

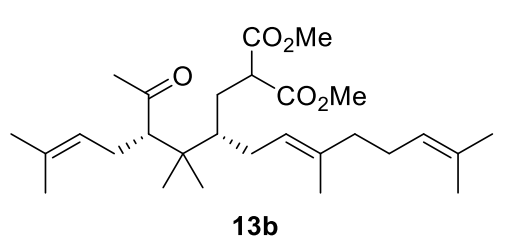

13b<smiles>CC(=O)CC(CC=C(C)C)C(CC=C(C)C)C(C)(C)C(CC=C(C)C)C(C)=O</smiles>

13b'<smiles>CC(=O)OC(C/C=C(/C)CCC=C(C)C)CC(CC(C(C)=O)C(C)(C)C)[C@@H](CC=C(C)C)C(C)=O</smiles>

13b<smiles>CC(=O)C[C@@H](CC=C(C)C)C(C)(C)C(CC(=CCC(C)=CCC=C(C)C)C(C)=O)CC(CC=C(C)C)C(C)=O</smiles>

13b'

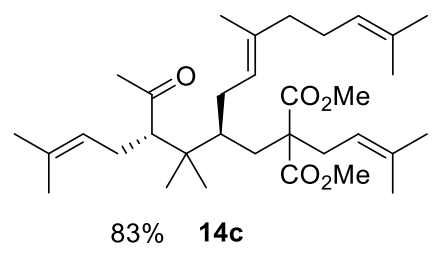

prenyl bromide $\mathrm{NaH}, \mathrm{DMSO}$

$14 c$

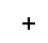<smiles>COC(=O)[C@@H](C=C(C)C)OC(=O)C(C)(CC(CC=C(C)C)C(C)(C)[C@@H](CC=C(C)CCC=C(C)C)C(C)=O)C(=O)OC</smiles>

$75 \% \quad 14 d$

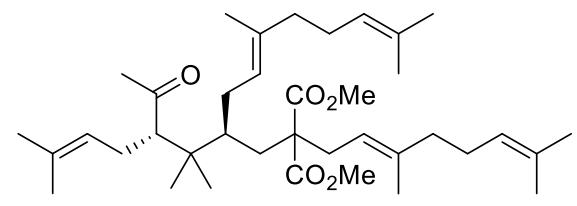

$14 \mathrm{e}$

geranyl bromide $\mathrm{NaH}, \mathrm{DMSO}, 80 \%$

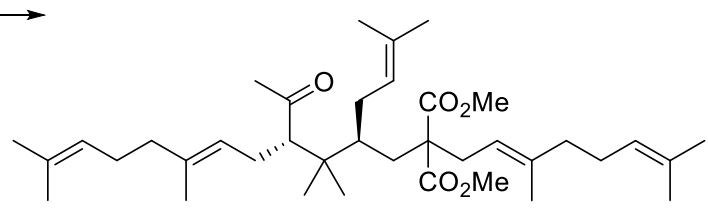

$14 f$

Compounds $\mathbf{1 4 c} / \mathbf{1 4 d}$ were prepared according to the general procedure that used for $\mathbf{1 4 a}$ with compounds $\mathbf{1 3 b} / \mathbf{1 3 b}$, (4.3 g, $\left.9.3 \mathrm{mmol}, \mathbf{1 3 b} / \mathbf{1 3 b} \mathbf{b}^{\prime}=1.5: 1\right), \mathrm{NaH}(60 \%$ in mineral oil, $558 \mathrm{mg}, 14.0 \mathrm{mmol})$ and prenyl bromide $(95 \%, 1.7 \mathrm{~mL}$, $14.0 \mathrm{mmol})$. The residue was purified by column chromatography over silica gel to yield the desired products $14 \mathbf{c}(2.6 \mathrm{~g}$, $83 \%)$ and $14 \mathrm{~d}(1.5 \mathrm{~g}, 75 \%)$ as pale yellow oils. $14 \mathrm{c}: R_{f}=0.45$ (petroleum ether/ethyl acetate, $\left.10: 1\right) .{ }^{1} \mathrm{H} \mathrm{NMR} \mathrm{(600} \mathrm{MHz,}$ $\left.\mathrm{CDCl}_{3}\right) \delta 5.07-5.06(\mathrm{~m}, 2 \mathrm{H}), 4.99-4.91(\mathrm{~m}, 2 \mathrm{H}), 3.67(\mathrm{~s}, 3 \mathrm{H}), 3.66(\mathrm{~s}, 3 \mathrm{H}), 2.76(\mathrm{dd}, J=13.9 \mathrm{~Hz}, 1 \mathrm{H}), 2.71-2.62(\mathrm{~m}$, $1 \mathrm{H}), 2.59-2.53(\mathrm{~m}, 1 \mathrm{H}), 2.40-2.32(\mathrm{~m}, 1 \mathrm{H}), 2.20(\mathrm{~d}, J=15.8 \mathrm{~Hz}, 1 \mathrm{H}), 2.09(\mathrm{~s}, 3 \mathrm{H}), 2.04-1.79(\mathrm{~m}, 9 \mathrm{H}), 1.69(\mathrm{~s}, 3 \mathrm{H})$ $1.66(\mathrm{~s}, 3 \mathrm{H}), 1.63(\mathrm{~s}, 3 \mathrm{H}), 1.60(\mathrm{~s}, 3 \mathrm{H}), 1.59(\mathrm{~s}, 3 \mathrm{H}), 1.56(\mathrm{~s}, 3 \mathrm{H}), 1.54(\mathrm{~s}, 3 \mathrm{H}), 0.87(\mathrm{~s}, 3 \mathrm{H}), 0.84(\mathrm{~s}, 3 \mathrm{H}) .{ }^{13} \mathrm{C}$ NMR $(151$ $\left.\mathrm{MHz}, \mathrm{CDCl}_{3}\right) \delta 213.5,172.6,172.5,135.8,133.7,133.2,131.4,125.2,124.5,122.4,117.8,57.4,57.0,52.5,52.3,40.1$, $39.9,34.7,34.0,31.9,29.9,28.7,27.3,26.6,26.1,26.0,25.9,22.1,21.5,18.1,17.9,17.8,16.4$. IR (thin film) $v_{\max } 2966$, 2924, 1735, 1435, 1218, $1172 \mathrm{~cm}^{-1}$. HRESIMS calcd for $\mathrm{C}_{33} \mathrm{H}_{55} \mathrm{O}_{5}[\mathrm{M}+\mathrm{H}]^{+} 531.4044$, found 531.4068. 14d: $R_{f}=0.55$ 
(petroleum ether/ethyl acetate, 10:1). ${ }^{1} \mathrm{H}$ NMR (400 MHz, $\left.\mathrm{CDCl}_{3}\right) \delta 5.04(\mathrm{~m}, 2 \mathrm{H}), 4.99-4.94(\mathrm{~m}, 2 \mathrm{H}), 3.67(\mathrm{~s}, 3 \mathrm{H}), 3.66$ (s, 3H), $2.76(\mathrm{dd}, J=13.8 \mathrm{~Hz}, 1 \mathrm{H}), 2.70-2.64(\mathrm{~m}, 1 \mathrm{H}), 2.56(\mathrm{dd}, J=15.4,6.6 \mathrm{~Hz}, 1 \mathrm{H}), 2.41-2.32(\mathrm{~m}, 1 \mathrm{H}), 2.20(\mathrm{~d}, J=$ $14.7 \mathrm{~Hz}, 1 \mathrm{H}), 2.07$ (s, 3H), $2.02-1.79(\mathrm{~m}, 9 \mathrm{H}), 1.68(\mathrm{~s}, 3 \mathrm{H}), 1.65(\mathrm{~s}, 3 \mathrm{H}), 1.64(\mathrm{~s}, 3 \mathrm{H}), 1.60(\mathrm{~s}, 3 \mathrm{H}), 1.57(\mathrm{~s}, 3 \mathrm{H}), 1.55(\mathrm{~s}$, $3 \mathrm{H}), 1.54(\mathrm{~s}, 3 \mathrm{H}), 0.88(\mathrm{~s}, 3 \mathrm{H}), 0.84(\mathrm{~s}, 3 \mathrm{H}) .{ }^{13} \mathrm{C} \mathrm{NMR}\left(101 \mathrm{MHz}, \mathrm{CDCl}_{3}\right) \delta 213.4,172.6,172.5,136.9,135.7,131.5$, 130.1, 125.1, 124.3, 122.3, 117.9, 57.4, 57.0, 52.5, 52.3, 40.14, 40.13, 40.0, 34.7, 34.0, 31.8, 28.8, 27.2, 26.8, 26.2, 25.9, 25.8, 22.2, 21.6, 18.14, 18.10, 17.8, 16.1. IR (thin film) $v_{\max } 2967,2922,1737,1711,1435,1218 \mathrm{~cm}^{-1}$. HRESIMS calcd for $\mathrm{C}_{33} \mathrm{H}_{55} \mathrm{O}_{5}[\mathrm{M}+\mathrm{H}]^{+}$531.4044, found 531.4048.

Compounds $\mathbf{1 4 e} / \mathbf{1 4 f} \mathbf{f}$ were prepared according to the general procedure that used for $\mathbf{1 4 a}$ with compounds $\mathbf{1 3 b} / \mathbf{1 3 b}$ ' (6 g, 12.9 mmol, 13b/13b' = 1.5:1), $\mathrm{NaH}(60 \%$ in mineral oil, $774 \mathrm{mg}, 19.3 \mathrm{mmol})$ and geranyl bromide $(96 \%, 4 \mathrm{~mL}, 19.3$ $\mathrm{mmol})$. The residue was purified by column chromatography over silica gel to yield the desired products $\mathbf{1 4 e} / \mathbf{1 4 f}(6.2 \mathrm{~g}$, $80 \%, \mathbf{1 4 e} / \mathbf{1 4 f}=1.2: 1)$ as pale yellow oils. $R_{f}=0.6$ (petroleum ether/ethyl acetate, $\left.10: 1\right) .{ }^{1} \mathrm{H}$ NMR $\left(400 \mathrm{MHz}, \mathrm{CDCl}_{3}\right) \delta$ $5.05-4.94(\mathrm{~m}, 5 \mathrm{H}), 3.66(\mathrm{~s}, 3 \mathrm{H}), 3.65(\mathrm{~s}, 3 \mathrm{H}), 2.76(\mathrm{~d}, J=11.3 \mathrm{~Hz}, 1 \mathrm{H}), 2.65-2.58(\mathrm{~m}, 2 \mathrm{H}), 2.36(\mathrm{dd}, J=11.2 \mathrm{~Hz}, 1 \mathrm{H})$, $2.18(\mathrm{~d}, 1 \mathrm{H}), 2.07(\mathrm{~d}, J=3.3 \mathrm{~Hz}, 4 \mathrm{H}), 2.03-1.79(\mathrm{~m}, 12 \mathrm{H}), 1.67-1.53(\mathrm{~m}, 24 \mathrm{H}), 0.85(\mathrm{dd}, J=12.0,2.2 \mathrm{~Hz}, 6 \mathrm{H}) .{ }^{13} \mathrm{C}$ NMR $\left(101 \mathrm{MHz}, \mathrm{CDCl}_{3}\right) \delta 213.4,213.3,172.50,172.46,139.43,139.37,136.9,133.6,133.2,131.63,131.62,131.5$, $131.4,130.1,125.33,125.25,124.5,124.3,124.1,122.3,117.8,117.7,57.5,57.4,57.0,56.9,52.5,52.3,40.3,40.14$, $40.11,40.0,39.9,34.72,34.68,34.24,34.19,31.79,31.76,29.0,28.8,27.3,27.2,26.83,26.81,26.77,26.6,25.92,25.88$, $25.83,25.80,22.14,22.07,21.6,21.5,18.2,17.9,17.82,17.79,17.78,16.40,16.36,16.1$. IR (thin film) $v_{\max } 2918,1732$, $1711,1435,1216,1106 \mathrm{~cm}^{-1}$. HRESIMS calcd for $\mathrm{C}_{38} \mathrm{H}_{62} \mathrm{NaO}_{5}[\mathrm{M}+\mathrm{Na}]^{+} 621.4489$, found 621.4527 .

\section{Table S1. Evaluation of the domino Dieckmann cyclization with $14 a^{a}$}

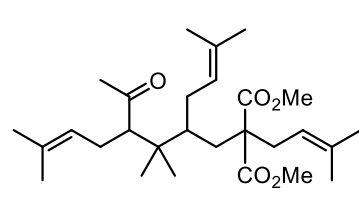

$14 \mathrm{a}$

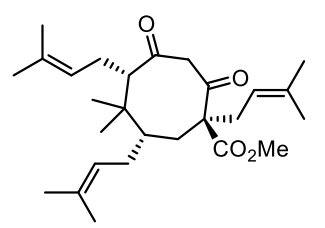

16

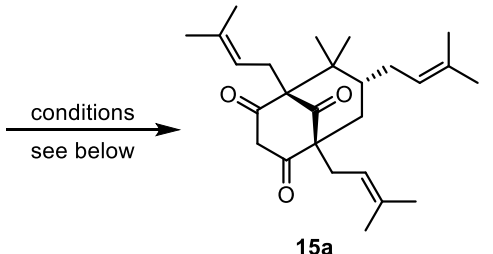

$15 \mathrm{a}$

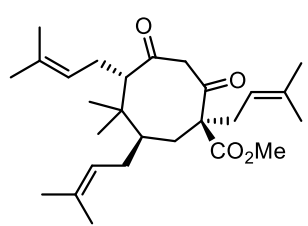

17

\begin{tabular}{|c|c|c|c|}
\hline entry & conditions (equiv) & solvent (temperature) & results, yield \\
\hline 1 & LiHMDS (5) & toluene $\left(75^{\circ} \mathrm{C}\right)$ & $\mathbf{1 6} / \mathbf{1 7}, 67 \%$ \\
\hline 2 & LiHMDS (5) & toluene (reflux) & $\mathbf{1 6} / \mathbf{1 7}, 48 \%$ \\
\hline 3 & LiHMDS (5) & toluene $\left(40{ }^{\circ} \mathrm{C}\right)$ & $\mathbf{1 6} / \mathbf{1 7}, 84 \%$ \\
\hline 4 & $\mathrm{KO}^{t} \mathrm{Bu}(5)$ & THF (reflux) & $-b$ \\
\hline 5 & $\mathrm{NaH}(5)$ & toluene (reflux) & $-b$ \\
\hline 6 & LiHMDS (5), HMPA (5) & toluene $\left(75^{\circ} \mathrm{C}\right)$ & $16 / 17,63 \%$ \\
\hline 7 & $\operatorname{LiHMDS}(5), \mathrm{TiCl}_{4}(0.1)$ & toluene $\left(75^{\circ} \mathrm{C}\right)$ & $-b$ \\
\hline 8 & LiHMDS (5), TMSOTf (0.1) & toluene $\left(75^{\circ} \mathrm{C}\right)$ & $-b$ \\
\hline 9 & LiHMDS (5), TMSCl (0.1) & toluene $\left(75^{\circ} \mathrm{C}\right)$ & $-b$ \\
\hline 10 & LiHMDS (5), $\mathrm{ZnCl}_{2}(0.1)$ & toluene $\left(75^{\circ} \mathrm{C}\right)$ & $-b$ \\
\hline 11 & LiHMDS (10) & toluene $\left(75^{\circ} \mathrm{C}\right)$ & $\mathbf{1 6} / \mathbf{1 7}, 46 \%$ \\
\hline
\end{tabular}




\begin{tabular}{|c|c|c|c|}
\hline 12 & NaHMDS (5) & toluene $\left(75^{\circ} \mathrm{C}\right)$ & $\mathbf{1 6} / \mathbf{1 7}, 10 \%$ \\
\hline 13 & $\mathrm{TiCl}_{4}(6) / \mathrm{Et}_{3} \mathrm{~N}(6)$ & DCE (reflux) & $-b$ \\
\hline 14 & $\mathrm{AlCl}_{3}(6) / \mathrm{Et}_{3} \mathrm{~N}(6)$ & DCE (reflux) & $-b$ \\
\hline 15 & $\mathrm{Me}_{3} \mathrm{Al}(10)$ & $\mathrm{CH}_{2} \mathrm{Cl}_{2} /$ heptane $(1 / 1)$ (reflux) & $-b$ \\
\hline 16 & $\mathrm{Me}_{3} \mathrm{Al}(4) / \mathrm{EtSH}(4)$ & $\mathrm{CH}_{2} \mathrm{Cl}_{2} /$ heptane $(1 / 1)$ (reflux) & $-b$ \\
\hline 17 & $\mathrm{Me}_{3} \mathrm{Al}(30) / \mathrm{EtSH}(30)$ & $\mathrm{CH}_{2} \mathrm{Cl}_{2} /$ heptane $(1 / 1)(\mathrm{rt})$ & $16 / 17(73 \%)$ \\
\hline 18 & $\mathrm{Me}_{3} \mathrm{Al}(10) / \mathrm{EtSH}(10)$ & $\mathrm{CH}_{2} \mathrm{Cl}_{2} /$ heptane $(1 / 1)(\mathrm{rt})$ & $\mathbf{1 6} / \mathbf{1 7}(61 \%)+\mathbf{1 5 a}(5 \%)$ \\
\hline 19 & $\mathrm{Me}_{3} \mathrm{Al}(30) / \mathrm{EtSH}(30)$ & $\mathrm{CH}_{2} \mathrm{Cl}_{2} /$ heptane $(1 / 1)$ (reflux) & $17(12 \%)+\mathbf{1 5 a}(45 \%)$ \\
\hline 20 & $\mathrm{Me}_{3} \mathrm{Al}(40) / \mathrm{EtSH}(40)$ & $\mathrm{CH}_{2} \mathrm{Cl}_{2} /$ heptane $(1 / 1)$ (reflux) & $17(<5 \%)+15 a(60 \%)$ \\
\hline 21 & $\mathrm{Al}(\mathrm{Me})_{3}(40) / \mathrm{PhSH}(40)$ & $\mathrm{CH}_{2} \mathrm{Cl}_{2} /$ heptane $(1 / 1)$ (reflux) & $15 \mathbf{a}(45 \%)$ \\
\hline 22 & $\mathrm{Al}(\mathrm{Me})_{3}(5) / \mathrm{EtSH}(5)$ & $\mathrm{CH}_{2} \mathrm{Cl}_{2} /$ heptane (10/1) (reflux) & 15a $(75 \%)$ \\
\hline 23 & $\mathrm{Al}(\mathrm{Me})_{3}(5) / \mathrm{EtSH}(5)$ & $\mathrm{CH}_{2} \mathrm{Cl}_{2} /$ heptane $(5 / 1)$ (reflux) & $15 \mathbf{a}(70 \%)$ \\
\hline 24 & $\mathrm{Al}(\mathrm{Me})_{3}(10) / \mathrm{EtSH}(10)$ & $\mathrm{CH}_{2} \mathrm{Cl}_{2} /$ heptane $(10 / 1)$ (reflux) & $15 \mathbf{a}(75 \%)$ \\
\hline 25 & $\mathrm{Me}_{2} \mathrm{AlCl}(40) / \mathrm{EtSH}(40)$ & $\mathrm{CH}_{2} \mathrm{Cl}_{2}$ (reflux) & $--^{c}$ \\
\hline 26 & $\mathrm{Me}_{2} \mathrm{AlCl}(10)$ & $\mathrm{CH}_{2} \mathrm{Cl}_{2}$ (reflux) & $--^{c}$ \\
\hline 27 & $\mathrm{AlCl}_{3}(20) / \mathrm{EtSH}(20)$ & $\mathrm{CH}_{2} \mathrm{Cl}_{2}$ (reflux) & $-c$ \\
\hline 28 & LiSEt (30) & THF (reflux) & $--^{b}$ \\
\hline 29 & $p$-Toluenesulfonic acid (0.2) & toluene (reflux) & $-^{c}$ \\
\hline
\end{tabular}

${ }^{a}$ Unless otherwise noted, the reaction was carried out with $14 a(46 \mathrm{mg}, 0.1 \mathrm{mmol})$ under $\mathrm{N}_{2}$ for 2 hours. The two major products $\mathbf{1 6} / \mathbf{1 7}$ was obtained at a ratio of $2 / 1$ to $3 / 1$ in entries $1-3,6$ and 11-12. The two major products $\mathbf{1 6} / \mathbf{1 7}$ was obtained at a ratio of $5 / 1$ to $6 / 1$ in entries $17-18$. A few other minor stereoisomers could also be detected in the mixture by ${ }^{1} \mathrm{H}$ NMR analysis in entries 1-3, 6 and 11-12. ${ }^{b}$ No reaction. ${ }^{c}$ Decomposition of 14a. HMPA $=$ Hexamethylphosphoric triamide. TMSOTf $=$ Trimethylsilyl trifluoromethanesulfonate. $\mathrm{TMSCl}=$ Chlorotrimethylsilane. $\mathrm{DCE}=1$, 2-Dichloroethane.

\section{Procedure for the base-catalyzed cascade Dieckmann condensation with $14 a$}
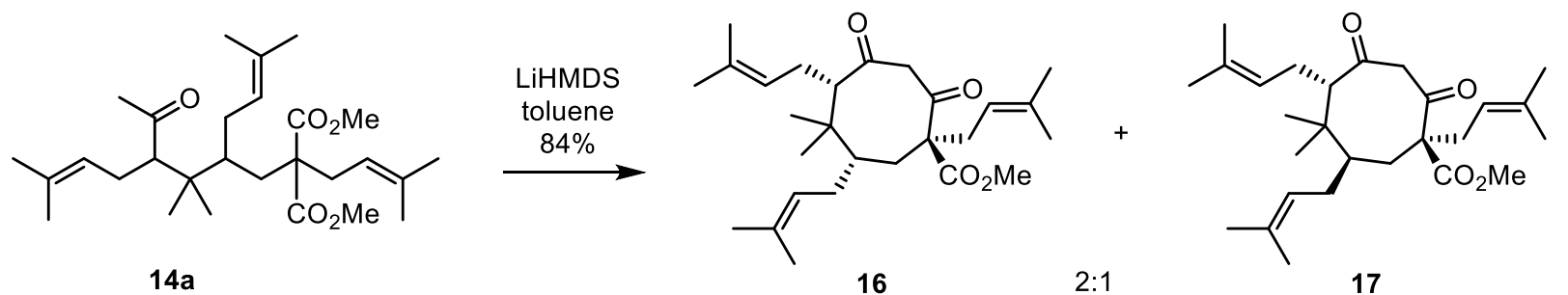

The compound 14a $(46.2 \mathrm{mg}, 0.1 \mathrm{mmol})$ was dissolved in $1.0 \mathrm{~mL}$ of toluene under $\mathrm{N}_{2}$. The solution was brought to $0{ }^{\circ} \mathrm{C}$, and a solution of lithium bis(trimethylsilyl)amide ( $1 \mathrm{M}$ in THF, $0.3 \mathrm{~mL}, 0.3 \mathrm{mmol}$ ) was added dropwise. Then the solution was stirred at $40{ }^{\circ} \mathrm{C}$ for 2 hours and quenched with $1 \mathrm{~N} \mathrm{HCl}$ after the solution was cooled to room temperature. The product was extracted from the aqueous layer with AcOEt $(3 \times 10 \mathrm{~mL})$, and the combined organic layers were dried over $\mathrm{Na}_{2} \mathrm{SO}_{4}$. The residue was purified by column chromatography over silica gel to yield the desired products 16 and $\mathbf{1 7}$ (32 $\mathrm{mg}, 84 \%, \mathbf{1 6} / \mathbf{1 7}=2: 1$ ) as pale yellow oils. If the reaction was stirred at $75{ }^{\circ} \mathrm{C}$ for 2 hours with lithium bis (trimethylsilyl) amide (1 M in THF, $0.5 \mathrm{~mL}, 0.5 \mathrm{mmol})$ in toluene $(1 \mathrm{~mL})$, only $25 \mathrm{mg}$ of 8 -membered products 16 and 17 $(58 \%, 2: 1)$ were prepared as pale yellow oils. Compound 16: $R_{f}=0.8$ (petroleum ether/ethyl acetate, 10:1). ${ }^{1} \mathrm{H}$ NMR $\left(400 \mathrm{MHz}, \mathrm{CDCl}_{3}\right) \delta 5.01(\mathrm{t}, J=7.0 \mathrm{~Hz}, 1 \mathrm{H}), 4.93-4.88(\mathrm{~m}, 2 \mathrm{H}), 3.73(\mathrm{~s}, 3 \mathrm{H}), 3.60(\mathrm{~d}, J=14.5 \mathrm{~Hz}, 1 \mathrm{H}), 3.15(\mathrm{~d}, J=$ $14.4 \mathrm{~Hz}, 1 \mathrm{H}), 2.62-2.57(\mathrm{~m}, 1 \mathrm{H}), 2.52-2.45(\mathrm{~m}, 2 \mathrm{H}), 2.39-2.28(\mathrm{~m}, 2 \mathrm{H}), 2.16-2.12(\mathrm{~m}, 1 \mathrm{H}), 2.00-1.93(\mathrm{~m}, 1 \mathrm{H})$, $1.78-1.70(\mathrm{~m}, 3 \mathrm{H}), 1.67(\mathrm{~s}, 3 \mathrm{H}), 1.65(\mathrm{~s}, 3 \mathrm{H}), 1.63(\mathrm{~s}, 3 \mathrm{H}), 1.61(\mathrm{~s}, 3 \mathrm{H}), 1.57(\mathrm{~s}, 3 \mathrm{H}), 1.55(\mathrm{~s}, 3 \mathrm{H}), 0.97(\mathrm{~s}, 3 \mathrm{H}), 0.88(\mathrm{~s}$, 
3H). ${ }^{13} \mathrm{C} \mathrm{NMR}\left(101 \mathrm{MHz}, \mathrm{CDCl}_{3}\right) \delta 208.4,202.9,171.5,135.9,134.7,131.6,124.2,120.8,118.0,66.5,61.0,60.4,52.1$, $45.4,42.4,37.6,36.1,32.6,27.9,27.2,26.11,26.08,25.9,18.2,18.0,17.7,15.8$. IR (thin film) $v_{\max } 2926,1726,1650$, 1448, 1224, $851 \mathrm{~cm}^{-1}$. HRESIMS calcd for $\mathrm{C}_{27} \mathrm{H}_{43} \mathrm{O}_{4}[\mathrm{M}+\mathrm{H}]^{+}$431.3156, found 431.3160. Compound 17: $R_{f}=0.7$ (petroleum ether/ethyl acetate, 10:1). ${ }^{1} \mathrm{H} \mathrm{NMR}\left(400 \mathrm{MHz}, \mathrm{CDCl}_{3}\right) \delta 4.99-4.91(\mathrm{~m}, 3 \mathrm{H}), 3.88(\mathrm{~d}, J=9.2 \mathrm{~Hz}, 1 \mathrm{H}), 3.66(\mathrm{~s}$, $3 \mathrm{H}), 3.30(\mathrm{~d}, J=9.3 \mathrm{~Hz}, 1 \mathrm{H}), 2.98(\mathrm{dd}, J=11.3,2.2 \mathrm{~Hz}, 1 \mathrm{H}), 2.77(\mathrm{dd}, J=15.2,7.7 \mathrm{~Hz}, 1 \mathrm{H}), 2.68(\mathrm{dd}, J=16.1,2.5 \mathrm{~Hz}$, $1 \mathrm{H}), 2.49-2.41(\mathrm{~m}, 1 \mathrm{H}), 2.25-2.16(\mathrm{~m}, 2 \mathrm{H}), 2.03-1.98(\mathrm{~m}, 1 \mathrm{H}), 1.91-1.80(\mathrm{~m}, 2 \mathrm{H}), 1.66(\mathrm{~s}, 3 \mathrm{H}), 1.62(\mathrm{~s}, 6 \mathrm{H}), 1.58$ $(\mathrm{s}, 3 \mathrm{H}), 1.56(\mathrm{~s}, 6 \mathrm{H}), 0.95(\mathrm{~s}, 3 \mathrm{H}), 0.87(\mathrm{~s}, 3 \mathrm{H}), 0.83-0.76(\mathrm{~m}, 1 \mathrm{H}) .{ }^{13} \mathrm{C} \mathrm{NMR}\left(101 \mathrm{MHz}, \mathrm{CDCl}_{3}\right) \delta 205.1,197.6,172.4$, 135.2, 134.0, 131.8, 124.7, 121.8, 118.2, 65.9, 61.5, 61.3, 52.6, 42.9, 42.6, 32.3, 31.9, 30.7, 27.4, 26.2, 25.94, 25.87, 21.2, 21.0, 18.1, 18.0, 17.8. IR (thin film) $v_{\max } 2925,1726,1450,1375,1218,1058 \mathrm{~cm}^{-1}$. HRESIMS calcd for $\mathrm{C}_{27} \mathrm{H}_{43} \mathrm{O}_{4}[\mathrm{M}+$ $\mathrm{H}]^{+} 431.3156$, found 431.3155 .

\section{General procedure for the $\mathrm{Me}_{3} \mathrm{Al} / \mathrm{EtSH}$ catalyzed cascade Dieckmann condensation}
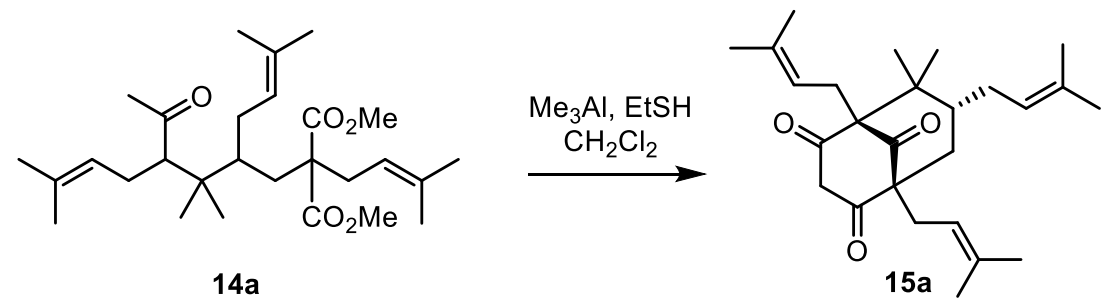

The ethanethiol $(97 \%, 40 \mu \mathrm{L}, 0.5 \mathrm{mmol})$ was dissolved in $1 \mathrm{~mL}$ of $\mathrm{CH}_{2} \mathrm{Cl}_{2}$ under $\mathrm{N}_{2}$. Trimethyl aluminium (1.0 M in heptane, $0.5 \mathrm{~mL}, 0.5 \mathrm{mmol}$ ) was added dropwise with emission of gas. The solution was stirred at room temperature for $0.5 \mathrm{~h}$. Then the solvents were removed under vacuum (with a cold trap in liq. $\mathrm{N}_{2}$ ) to give a white solid which was dissolved in $0.1 \mathrm{~mL}$ of dry heptane under $\mathrm{N}_{2}$. The substrate $14 \mathbf{a}(46 \mathrm{mg}, 0.1 \mathrm{mmol})$ in $1 \mathrm{~mL}$ of $\mathrm{CH}_{2} \mathrm{Cl}_{2} \mathrm{was}$ added dropwise and the reaction was stirred at $50{ }^{\circ} \mathrm{C}$ for 20 hours, quenched by $\mathrm{H}_{2} \mathrm{O}$ after the solution was cooled to room temperature. $1 \mathrm{~N} \mathrm{HCl}$ was added slowly into the white emulsion until the colloidal solution turned transparency and stirred for $10 \mathrm{~min}$. The product was extracted from the aqueous layer extracted with $\mathrm{CH}_{2} \mathrm{Cl}_{2}(3 \times 5 \mathrm{~mL})$. The organic layer was dried over $\mathrm{Na}_{2} \mathrm{SO}_{4}$ and purified via flash chromatography to give bicyclo[3.3.1]nonane product 15a as a yellow oil (30 mg, $75 \%) . R_{f}=0.15$ (petroleum ether/ethyl acetate, $\left.10: 1\right) .{ }^{1} \mathrm{H} \mathrm{NMR}\left(400 \mathrm{MHz}, \mathrm{CDCl}_{3}\right) \delta 5.11(\mathrm{t}, J=7.4 \mathrm{~Hz}, 1 \mathrm{H})$, $4.93(\mathrm{t}, J=7.4 \mathrm{~Hz}, 1 \mathrm{H}), 4.86(\mathrm{t}, J=6.8 \mathrm{~Hz}, 1 \mathrm{H}), 3.56(\mathrm{~d}, J=17.3 \mathrm{~Hz}, 1 \mathrm{H}), 2.96(\mathrm{~d}, J=17.3 \mathrm{~Hz}, 1 \mathrm{H}), 2.57-2.55(\mathrm{~m}, 2 \mathrm{H})$, $2.49(\mathrm{~d}, J=7.4 \mathrm{~Hz}, 2 \mathrm{H}), 2.19-2.15(\mathrm{~m}, 2 \mathrm{H}), 2.04(\mathrm{dd}, J=14.0,5.7 \mathrm{~Hz}, 1 \mathrm{H}), 1.67(\mathrm{~s}, 3 \mathrm{H}), 1.66(\mathrm{~s}, 3 \mathrm{H}), 1.65$ (s, $3 \mathrm{H})$, $1.61(\mathrm{~s}, 6 \mathrm{H}), 1.52(\mathrm{~s}, 3 \mathrm{H}), 1.47-1.41(\mathrm{~m}, 1 \mathrm{H}), 1.34-1.29(\mathrm{~m}, 1 \mathrm{H}), 1.26(\mathrm{~s}, 3 \mathrm{H}), 0.96(\mathrm{~s}, 3 \mathrm{H}) .{ }^{13} \mathrm{C} \mathrm{NMR}(101 \mathrm{MHz}, \mathrm{CDCl})$ $\delta$ 210.6, 203.0, 202.8, 136.6, 135.7, 133.8, 122.7, 118.2, 117.6, 70.3, 64.9, 62.7, 51.3, 46.5, 40.6, 31.3, 29.3, 27.3, 26.5, 26.20, 26.17, 26.0, 23.0, 18.11, 18.05, 18.0. IR (thin film) $v_{\max } 2963,2925,1729,1261,1091,800 \mathrm{~cm}^{-1}$. HRESIMS calcd for $\mathrm{C}_{26} \mathrm{H}_{39} \mathrm{O}_{3}[\mathrm{M}+\mathrm{H}]^{+}$399.2894, found 399.2899. Under the above conditions, this reaction has been run in $0.5 \mathrm{mmol}$ scale $(0.23 \mathrm{~g})$ to afford $15 \mathrm{a}$ in $72 \%$ yield.

If the cyclization reaction was run at $\mathrm{rt}$ instead of $50^{\circ} \mathrm{C}$, the 8 -membered ring intermediates $\mathbf{1 6} / \mathbf{1 7}(3 / 1)$ were obtained in $80 \%$ yield. Under these conditions, the other 8-membered ring intermediate 16' from $14 \mathbf{f}$ was obtained almost as one isomer in 73\% yield. Compound 16' (contaminated with few isomers generated from cis-geranyl group): ${ }^{1} \mathrm{H}$ NMR (600 $\left.\mathrm{MHz}, \mathrm{CDCl}_{3}\right) \delta 5.01-5.04(\mathrm{~m}, 5 \mathrm{H}), 3.92(\mathrm{~d}, J=9.0 \mathrm{~Hz}, 1 \mathrm{H}), 3.66(\mathrm{~s}, 3 \mathrm{H}), 3.28(\mathrm{~d}, J=9.0 \mathrm{~Hz}, 1 \mathrm{H}), 3.01-2.99(\mathrm{~m}, 1 \mathrm{H})$, 2.83-2.79 (m, 1H), $2.74-2.71(\mathrm{~m}, 1 \mathrm{H}), 2.51-2.43(\mathrm{~m}, 2 \mathrm{H}), 2.28-2.20(\mathrm{~m}, 2 \mathrm{H}), 2.02-1.83(\mathrm{~m}, 9 \mathrm{H}), 1.68(\mathrm{~s}, 3 \mathrm{H}), 1.67$ $(\mathrm{s}, 3 \mathrm{H}), 1.63(\mathrm{~s}, 6 \mathrm{H}), 1.59(\mathrm{~s}, 6 \mathrm{H}), 1.55(\mathrm{~s}, 6 \mathrm{H}), 1.25-1.23(\mathrm{~m}, 2 \mathrm{H}), 0.97(\mathrm{~s}, 3 \mathrm{H}), 0.86(\mathrm{~s}, 3 \mathrm{H}) .{ }^{13} \mathrm{C} \mathrm{NMR}(151 \mathrm{MHz}$, $\left.\mathrm{CDCl}_{3}\right) \delta 205.1,197.2,172.2,139.0,135.0,134.0,131.6,131.6,124.7,124.3,124.2,121.8,118.3,77.4,77.2,66.0,61.5$, 61.5, 52.6, 43.0, 42.7, 40.1, 39.9, 32.3, 31.8, 30.4, 27.4, 26.7, 26.5, 25.9, 25.9, 25.8, 21.2, 21.1, 17.8, 17.8, 16.4, 16.2. HRESIMS calcd for $\mathrm{C}_{37} \mathrm{H}_{59} \mathrm{O}_{4}[\mathrm{M}+\mathrm{H}]^{+} 567.4413$, found 567.4416 . 


\section{${ }^{1} \mathrm{H}$ NMR analysis of the mixed $\mathrm{Me}_{3} \mathrm{Al}$ and $\mathrm{EtSH}$ performed in $\mathrm{CD}_{2} \mathrm{Cl}_{2}$}

To gain more insight into the significance of this reaction, the active species of the domino Dieckmann reaction were then verified. First, the ${ }^{1} \mathrm{H}$ NMR spectroscopic analysis in $\mathrm{CD}_{2} \mathrm{Cl}_{2}$ indicated that a new quartet at $2.71 \mathrm{ppm}$ and a new single at $-0.49 \mathrm{ppm}$ emerged after mixing $\mathrm{Me}_{3} \mathrm{Al}$ with $\mathrm{EtSH}$ in ratios of 1:1 or 1:3. Their integrals were approximately 1:3, which indicated the generation of $\mathrm{Me}_{2} \mathrm{AlSEt}$ rather than $\mathrm{Al}(\mathrm{SEt})_{3}$ as the active Lewis acidic species. To the best of our knowledge, $\mathrm{Me}_{2} \mathrm{AlSEt}$ has been shown to be an excellent reagent for transthioesterifications. This is the first study to demonstrate that $\mathrm{Me}_{2} \mathrm{AlSEt}$ can be used as a Lewis acid and promote a Dieckmann cyclization.

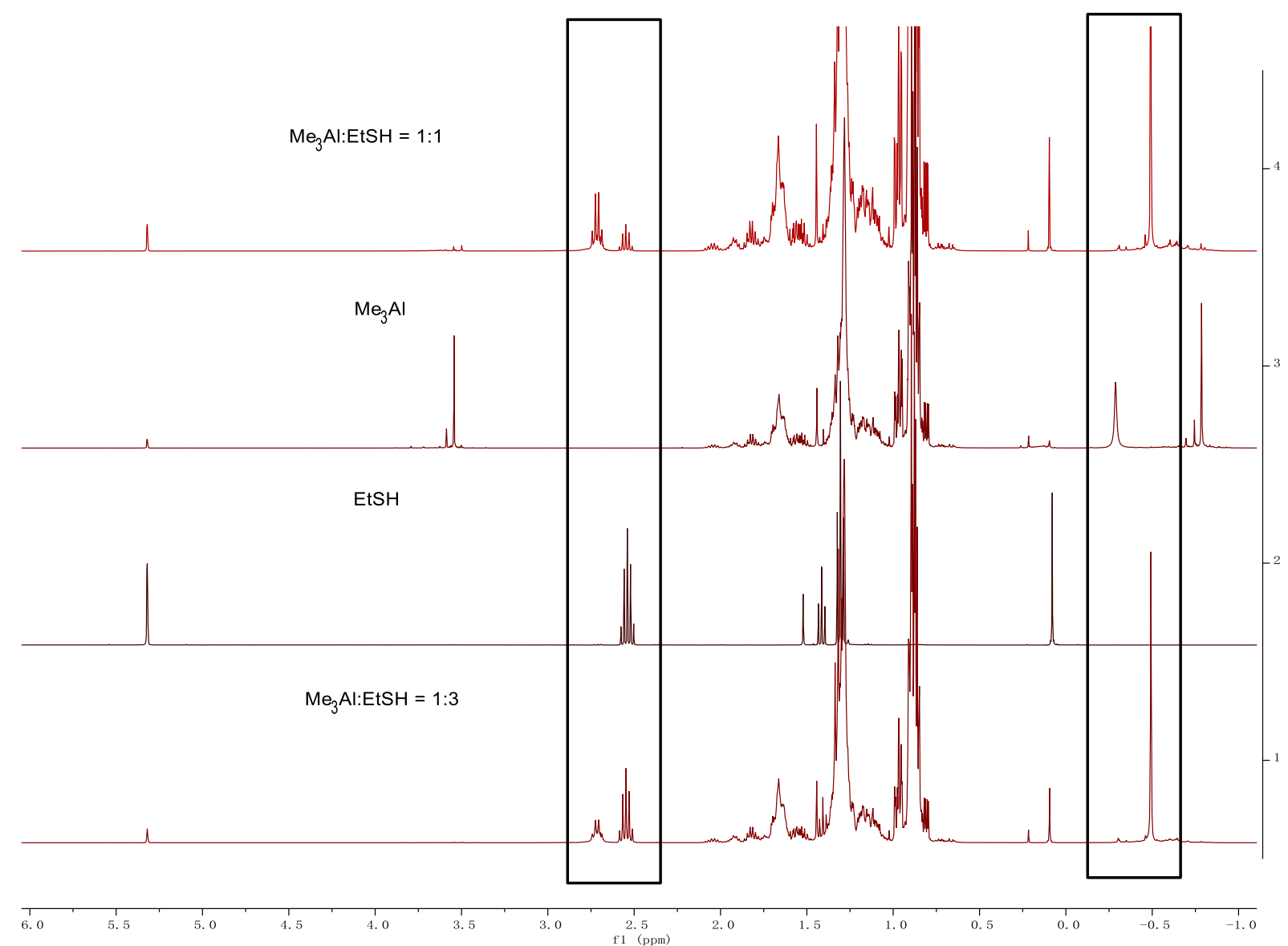

Procedures for the ${ }^{1} \mathrm{H}$ NMR analysis

(1) $\mathrm{Me}_{3} \mathrm{Al}: \mathrm{EtSH}=1: 1$. The ethanethiol $(97 \%, 33 \mu \mathrm{L}, 0.43 \mathrm{mmol})$ was dissolved in $1 \mathrm{~mL}$ of $\mathrm{CD}_{2} \mathrm{Cl}_{2}$ under $\mathrm{N}_{2}$, and trimethylaluminium (1.0 M in heptane, $0.43 \mathrm{~mL}, 0.43 \mathrm{mmol}$ ) was added dropwise with emission of gas. The solution was stirred at room temperature for $0.5 \mathrm{~h}$ and then analyzed by ${ }^{1} \mathrm{H}$ NMR directly.

(2) $\mathrm{Me}_{3} \mathrm{Al}$ : trimethylaluminium $(1.0 \mathrm{M}$ in heptane, $65 \mu \mathrm{L})$ in $0.5 \mathrm{~mL}$ of $\mathrm{CD}_{2} \mathrm{Cl}_{2}$.

(3) EtSH: Ethanethiol $(97 \%, 5 \mu \mathrm{L})$ in $0.5 \mathrm{~mL}$ of $\mathrm{CD}_{2} \mathrm{Cl}_{2}$.

(4) $\mathrm{Me}_{3} \mathrm{Al}: \mathrm{EtSH}=1: 3$. The ethanethiol $(97 \%, 100 \mu \mathrm{L}, 1.29 \mathrm{mmol})$ and trimethylaluminium $(1.0 \mathrm{M}$ in heptane, $0.43 \mathrm{~mL}$, $0.43 \mathrm{mmol}$ ) in $1 \mathrm{~mL}$ of $\mathrm{CD}_{2} \mathrm{Cl}_{2}$ was stirred at room temperature for $0.5 \mathrm{~h}$ and then analyzed by ${ }^{1} \mathrm{H} \mathrm{NMR}$.

Notes: As the generated $\mathrm{Me}_{2} \mathrm{AlSEt}$ was not soluble in $\mathrm{CD}_{2} \mathrm{Cl}_{2}$, the effort to remove the heptane after the reaction failed. Herein the ${ }^{1} \mathrm{H}$ NMR was taken with heptane which shown the signals at the range of 0.5-2.0 ppm. 


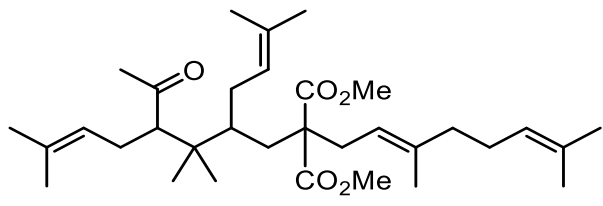

14b

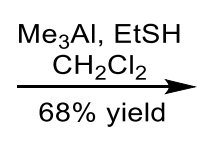

$68 \%$ yield

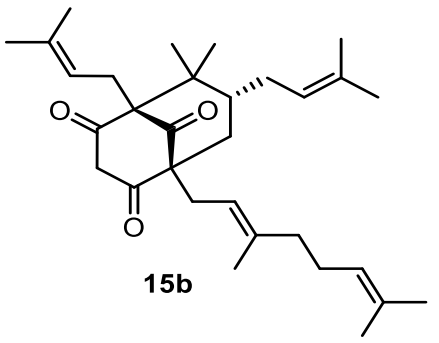

Compound 15b was prepared according to the general procedure that used for $\mathbf{1 5 a}$ with $\mathbf{1 4 b}(106 \mathrm{mg}, 0.2 \mathrm{mmol})$ to afford a yellow oil $(64 \mathrm{mg}, 68 \%) . R_{f}=0.15$ (petroleum ether/ethyl acetate, 10:1). ${ }^{1} \mathrm{H} \mathrm{NMR}\left(600 \mathrm{MHz}, \mathrm{CDCl}_{3}\right) \delta 5.13(\mathrm{t}$, $J=7.6 \mathrm{~Hz}, 1 \mathrm{H}), 5.01(\mathrm{t}, J=6.8 \mathrm{~Hz}, 1 \mathrm{H}), 4.95(\mathrm{t}, J=6.4 \mathrm{~Hz}, 1 \mathrm{H}), 4.86(\mathrm{t}, J=6.0 \mathrm{~Hz}, 1 \mathrm{H}), 3.55(\mathrm{~d}, J=17.1 \mathrm{~Hz}, 1 \mathrm{H}), 2.94$ $(\mathrm{d}, J=17.1 \mathrm{~Hz}, 1 \mathrm{H}), 2.57-2.54(\mathrm{~m}, 2 \mathrm{H}), 2.49(\mathrm{~d}, J=7.4 \mathrm{~Hz}, 2 \mathrm{H}), 2.18(\mathrm{t}, J=14.6 \mathrm{~Hz}, 2 \mathrm{H}), 2.08-1.95(\mathrm{~m}, 5 \mathrm{H}), 1.66(\mathrm{~s}$, $3 \mathrm{H}), 1.64(\mathrm{~s}, 3 \mathrm{H}), 1.63(\mathrm{~s}, 3 \mathrm{H}), 1.60(\mathrm{~s}, 6 \mathrm{H}), 1.56(\mathrm{~s}, 3 \mathrm{H}), 1.51(\mathrm{~s}, 3 \mathrm{H}), 1.48-1.39(\mathrm{~m}, 1 \mathrm{H}), 1.34-1.29(\mathrm{~m}, 1 \mathrm{H}), 1.26(\mathrm{~s}$, $3 \mathrm{H}), 0.96(\mathrm{~s}, 3 \mathrm{H}) .{ }^{13} \mathrm{C} \mathrm{NMR}\left(101 \mathrm{MHz}, \mathrm{CDCl}_{3}\right) \delta 210.4,202.8,139.2,136.4,133.6,131.4,124.1,122.6,118.0,117.5$, 70.2, 64.7, 62.9, 51.2, 46.5, 40.6, 40.0, 40.1, 31.2, 29.2, 27.1, 26.6, 26.4, 26.1, 25.8, 25.7, 23.0, 17.91, 17.87, 17.7, 16.3. IR (thin film) $v_{\max } 2925,1711,1593,1454,1376,1243 \mathrm{~cm}^{-1}$. HRESIMS calcd for $\mathrm{C}_{31} \mathrm{H}_{47} \mathrm{O}_{3}[\mathrm{M}+\mathrm{H}]^{+} 467.3530$, found 467.3544 .<smiles>COC(CC=C(C)C)(CC(CC=C(C)CCC=C(C)C)C(C)(C)C(CC=C(C)C)[C@@H](C)C(C)=O)C(C)(C)O</smiles>

$14 c$

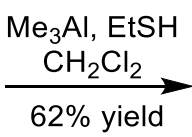

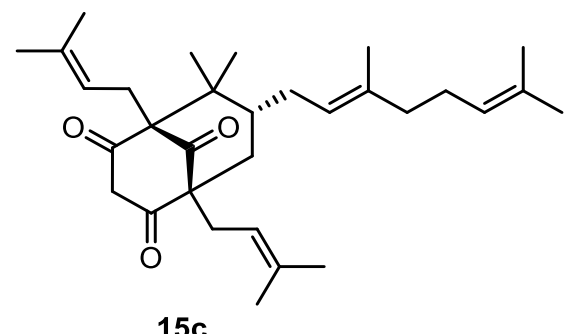

$15 c$

Compound 15c was prepared according to the general procedure that used for 15a with 14c $(106 \mathrm{mg}, 0.2 \mathrm{mmol})$, at $50{ }^{\circ} \mathrm{C}$ for 24 hours to afford a yellow oil $(58 \mathrm{mg}, 62 \%) . R_{f}=0.2$ (petroleum ether/ethyl acetate, 10:1). ${ }^{1} \mathrm{H}$ NMR (400 $\left.\mathrm{MHz}, \mathrm{CDCl}_{3}\right) \delta 5.12-5.04(\mathrm{~m}, 2 \mathrm{H}), 4.90(\mathrm{~m}, 2 \mathrm{H}), 3.57(\mathrm{~d}, J=17.2 \mathrm{~Hz}, 1 \mathrm{H}), 2.96(\mathrm{~d}, J=17.2 \mathrm{~Hz}, 1 \mathrm{H}), 2.61-2.55(\mathrm{~m}$, 2H), $2.48(\mathrm{~d}, J=7.3 \mathrm{~Hz}, 2 \mathrm{H}), 2.21-2.15(\mathrm{~m}, 2 \mathrm{H}), 2.07-2.02(\mathrm{~m}, 3 \mathrm{H}), 1.98-1.95(\mathrm{~m}, 2 \mathrm{H}), 1.67(\mathrm{~s}, 3 \mathrm{H}), 1.654(\mathrm{~s}, 3 \mathrm{H})$, $1.648(\mathrm{~s}, 3 \mathrm{H}), 1.61(\mathrm{~s}, 6 \mathrm{H}), 1.60(\mathrm{~s}, 3 \mathrm{H}), 1.51(\mathrm{~s}, 3 \mathrm{H}), 1.49-1.42(\mathrm{~m}, 1 \mathrm{H}), 1.36-1.30(\mathrm{~m}, 1 \mathrm{H}), 1.27(\mathrm{~s}, 3 \mathrm{H}), 0.97(\mathrm{~s}, 3 \mathrm{H})$. ${ }^{13} \mathrm{C} \mathrm{NMR}\left(101 \mathrm{MHz}, \mathrm{CDCl}_{3}\right) \delta 210.6,203.0,202.8,137.3,136.5,135.7,131.6,124.3,122.9,118.2,117.6,70.4,64.8$, $62.9,51.4,46.6,40.9,39.9,31.5,29.3,27.3,26.7,26.6,26.20,26.16,25.9,23.1,18.11,18.06,17.9$, 16.3. IR (thin film) $v_{\max } 2970,2925,1730,1705,1621,1595,1454,1375,1395,1238 \mathrm{~cm}^{-1}$. HRESIMS calcd for $\mathrm{C}_{31} \mathrm{H}_{47} \mathrm{O}_{3}[\mathrm{M}+\mathrm{H}]^{+}$ 467.3530 , found 467.3521 .
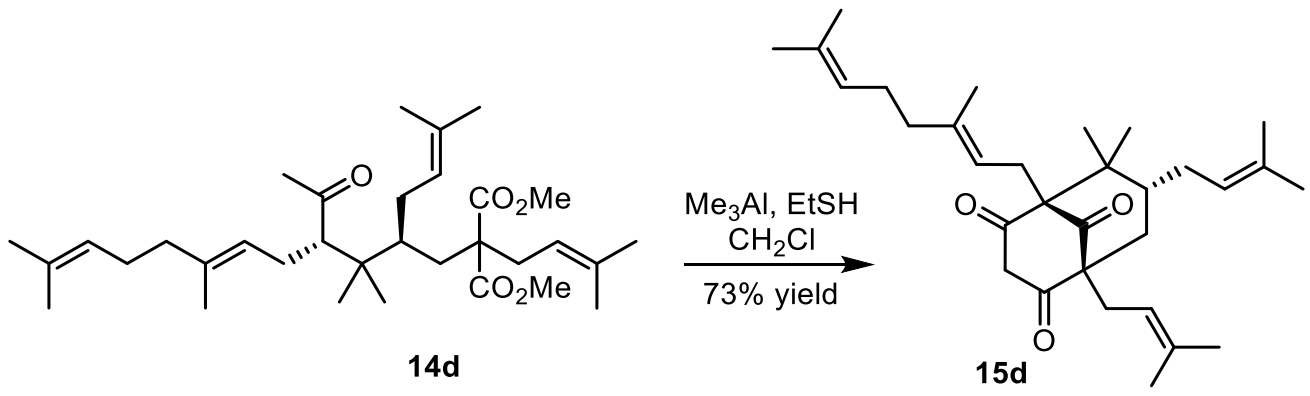

Compound 15d was prepared according to the general procedure that used for 15a with 14d (106 mg, $0.2 \mathrm{mmol})$, at $50{ }^{\circ} \mathrm{C}$ for 24 hours to afford a yellow oil $(68 \mathrm{mg}, 73 \%) . R_{f}=0.2$ (petroleum ether/ethyl acetate, 10:1). ${ }^{1} \mathrm{H}$ NMR (400 $\left.\mathrm{MHz}, \mathrm{CDCl}_{3}\right) \delta 5.10(\mathrm{t}, J=7.3 \mathrm{~Hz}, 1 \mathrm{H}), 4.95(\mathrm{t}, J=6.7 \mathrm{~Hz}, 2 \mathrm{H}), 4.86(\mathrm{t}, J=6.7 \mathrm{~Hz}, 1 \mathrm{H}), 3.54(\mathrm{~d}, J=17.2 \mathrm{~Hz}, 1 \mathrm{H}), 2.97$ $(\mathrm{d}, J=17.2 \mathrm{~Hz}, 1 \mathrm{H}), 2.61-2.56(\mathrm{~m}, 2 \mathrm{H}), 2.48(\mathrm{~d}, J=7.4 \mathrm{~Hz}, 2 \mathrm{H}), 2.17(\mathrm{dd}, J=17.0 \mathrm{~Hz}, 2 \mathrm{H}), 2.06-2.01(\mathrm{~m}, 2 \mathrm{H}), 1.94$ 
$-1.92(\mathrm{~m}, 1 \mathrm{H}),[1.67(\mathrm{~s}), 1.65(\mathrm{~s})](9 \mathrm{H}), 1.61(\mathrm{~s}, 6 \mathrm{H}), 1.69-1.60(\mathrm{~m}, 1 \mathrm{H}), 1.55(\mathrm{~s}, 3 \mathrm{H}), 1.51(\mathrm{~s}, 3 \mathrm{H}), 1.47-1.40(\mathrm{~m}, 2 \mathrm{H})$, $1.34-1.29(\mathrm{~m}, 1 \mathrm{H}), 1.26(\mathrm{~s}, 3 \mathrm{H}), 0.97(\mathrm{~s}, 3 \mathrm{H}) .{ }^{13} \mathrm{C} \mathrm{NMR}\left(101 \mathrm{MHz}, \mathrm{CDCl}_{3}\right) \delta 210.5,202.9,202.8,140.1,135.7,133.7$, $131.8,124.3,122.8,118.2,117.6,70.4,64.9,62.8,51.3,46.5,40.8,40.1,31.4,29.4,27.2,26.6,26.5,26.2,26.0,25.8$, 23.0, 18.1, 18.0, 17.8, 16.4. IR (thin film) $v_{\max } 2967,2924,1732,1712,1592,1445,1376,1235 \mathrm{~cm}^{-1}$. HRESIMS calcd for $\mathrm{C}_{31} \mathrm{H}_{47} \mathrm{O}_{3}[\mathrm{M}+\mathrm{H}]^{+}$467.3530, found 467.3526.
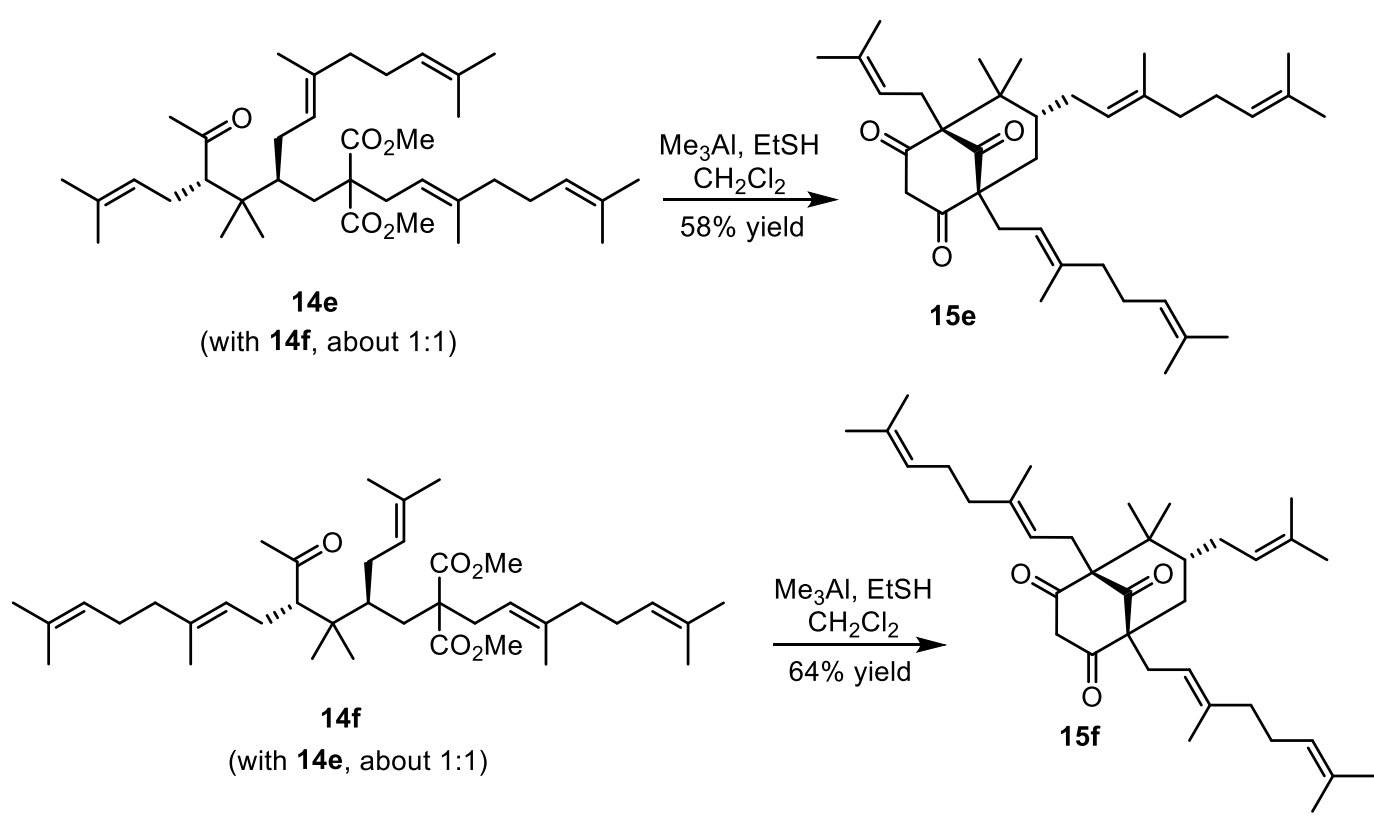

Compound 15e and $15 \mathrm{f}$ was prepared according to the general procedure that used for 15a with the mixture of 14e and $\mathbf{1 4 f}(240 \mathrm{mg}, 0.4 \mathrm{mmol}, \mathbf{1 4} / \mathbf{1 4 f}=1.2: 1)$ at $50{ }^{\circ} \mathrm{C}$ for 24 hours to afford $\mathbf{1 5 e}(62 \mathrm{mg}, 58 \%)$ and $\mathbf{1 5 f}(68 \mathrm{mg}, 64 \%)$ as yellow oils. Compound 15e: $R_{f}=0.2$ (petroleum ether/ethyl acetate, 10:1). ${ }^{1} \mathrm{H}$ NMR $\left(600 \mathrm{MHz}, \mathrm{CDCl}_{3}\right) \delta 5.13(\mathrm{t}, J=$ $6.9 \mathrm{~Hz}, 1 \mathrm{H}), 5.06(\mathrm{t}, J=7.0 \mathrm{~Hz}, 1 \mathrm{H}), 5.00(\mathrm{t}, J=6.8 \mathrm{~Hz}, 1 \mathrm{H}), 4.93(\mathrm{t}, J=6.4 \mathrm{~Hz}, 1 \mathrm{H}), 4.87$ (t, $J=6.2 \mathrm{~Hz}, 1 \mathrm{H}), 3.56(\mathrm{~d}, J$ $=17.0 \mathrm{~Hz}, 1 \mathrm{H}), 2.94(\mathrm{~d}, J=17.0 \mathrm{~Hz}, 1 \mathrm{H}), 2.60-2.52(\mathrm{~m}, 2 \mathrm{H}), 2.49(\mathrm{~d}, J=7.4 \mathrm{~Hz}, 2 \mathrm{H}), 2.22-2.16(\mathrm{~m}, 2 \mathrm{H}), 2.08-2.00$ (m, 6H), $1.96-1.94(\mathrm{~d}, J=7.2 \mathrm{~Hz}, 3 \mathrm{H}), 1.67$ (s, 3H), $1.64(\mathrm{~s}, 3 \mathrm{H}), 1.63(\mathrm{~s}, 3 \mathrm{H}), 1.61(\mathrm{~s}, 3 \mathrm{H}), 1.60(\mathrm{~s}, 3 \mathrm{H}), 1.59(\mathrm{~s}, 3 \mathrm{H})$, $1.56(\mathrm{~s}, 3 \mathrm{H}), 1.50(\mathrm{~s}, 3 \mathrm{H}), 1.48-1.42(\mathrm{~m}, 1 \mathrm{H}), 1.35-1.32(\mathrm{~m}, 1 \mathrm{H}), 1.27(\mathrm{~s}, 3 \mathrm{H}), 0.97(\mathrm{~s}, 3 \mathrm{H}) .{ }^{13} \mathrm{C}$ NMR $(101 \mathrm{MHz}$, $\left.\mathrm{CDCl}_{3}\right) \delta 210.6,202.91,202.89,139.3,137.3,136.5,131.57,131.55,124.32,124.26,122.9,118.1,117.6,70.4,64.8$, 63.2, 51.5, 46.7, 41.1, 40.1, 39.9, 31.5, 29.4, 27.3, 26.8, 26.7, 26.6, 26.2, 25.9, 23.2, 18.1, 17.84, 17.80, 16.4, 16.3. IR (thin film) $v_{\max } 2968,2924,1731,1711,1592,1446,1375,1242 \mathrm{~cm}^{-1}$. HRESIMS calcd for $\mathrm{C}_{36} \mathrm{H}_{55} \mathrm{O}_{3}[\mathrm{M}+\mathrm{H}]^{+} 535.4146$, found 535.4189. Compound 15f: $R_{f}=0.3$ (petroleum ether/ethyl acetate, 10:1). ${ }^{1} \mathrm{H}$ NMR (600 MHz, $\left.\mathrm{CDCl}_{3}\right) \delta 5.13$ (t, $J=$ $7.6 \mathrm{~Hz}, 1 \mathrm{H}), 5.01(\mathrm{t}, J=6.8 \mathrm{~Hz}, 1 \mathrm{H}), 4.95(\mathrm{t}, J=6.4 \mathrm{~Hz}, 2 \mathrm{H}), 4.86(\mathrm{t}, J=6.0 \mathrm{~Hz}, 1 \mathrm{H}), 3.54(\mathrm{~d}, J=17.1 \mathrm{~Hz}, 1 \mathrm{H}), 2.96(\mathrm{~d}$, $J=17.1 \mathrm{~Hz}, 1 \mathrm{H}), 2.60-2.53(\mathrm{~m}, 2 \mathrm{H}), 2.49(\mathrm{~d}, J=7.4 \mathrm{~Hz}, 2 \mathrm{H}), 2.20-2.15(\mathrm{~m}, 2 \mathrm{H}), 2.07-1.89(\mathrm{~m}, 9 \mathrm{H}), 1.66(\mathrm{~s}, 3 \mathrm{H})$, $1.645(\mathrm{~s}, 3 \mathrm{H}), 1.639(\mathrm{~s}, 3 \mathrm{H}), 1.62(\mathrm{~s}, 3 \mathrm{H}), 1.61(\mathrm{~s}, 3 \mathrm{H}), 1.56(\mathrm{~s}, 3 \mathrm{H}), 1.55(\mathrm{~s}, 3 \mathrm{H}), 1.51(\mathrm{~s}, 3 \mathrm{H}), 1.47-1.41(\mathrm{~m}, 1 \mathrm{H}), 1.34-$ $1.30(\mathrm{~m}, 1 \mathrm{H}), 1.26(\mathrm{~s}, 3 \mathrm{H}), 0.96(\mathrm{~s}, 3 \mathrm{H}) .{ }^{13} \mathrm{C} \mathrm{NMR}\left(101 \mathrm{MHz}, \mathrm{CDCl}_{3}\right) \delta 210.5,202.9,202.8,184.2,140.1,139.4,133.7$, 131.7, 131.6, 124.23, 124.21, 122.8, 118.1, 117.6, 70.4, 64.9, 63.0, 51.3, 46.6, 40.7, 40.2, 40.1, 31.3, 29.4, 27.2, 26.9, $26.7,26.5,25.94,25.85,23.1,18.0,17.8,16.4,16.3$. IR (thin film) $v_{\max } 2968,2924,1731,1712,1592,1446,1375,1240$ $\mathrm{cm}^{-1}$. HRESIMS calcd for $\mathrm{C}_{36} \mathrm{H}_{55} \mathrm{O}_{3}[\mathrm{M}+\mathrm{H}]^{+}$535.4146, found 535.4188. 
Completion of total synthesis of epi-clusianone, garcimultiflorone H, garcimultiflorone I, guttiferone I, oblongifolin A, oblongifolin D and anologues

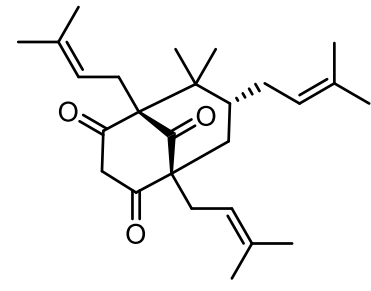

$15 a$

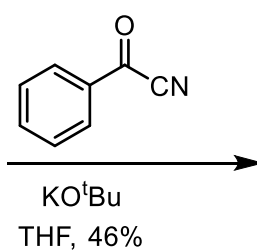

THF, $46 \%$

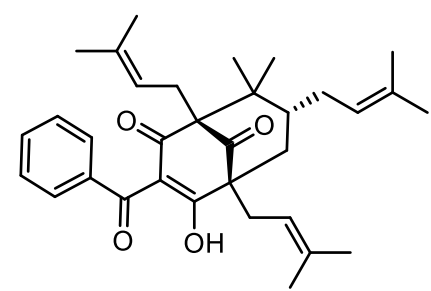

epi-clusianone (1)

The KO $\mathrm{Bu}$ (17 mg, $0.15 \mathrm{mmol})$, 4-dimethylaminopyridine (6 mg, $0.05 \mathrm{mmol})$ and compound $15 \mathrm{a}(20 \mathrm{mg}, 0.05$ mmol) were dissolved in $3 \mathrm{~mL}$ of THF under $\mathrm{N}_{2}$ and stirred at $0{ }^{\circ} \mathrm{C}$ for $10 \mathrm{~min}$. After benzoyl cyanide $(13 \mathrm{mg}, 0.1 \mathrm{mmol})$ was added, the mixture was warmed up to room temperature and stirred for 2 hours. The reaction was quenched with saturated solution of $\mathrm{NH}_{4} \mathrm{Cl}(5 \mathrm{~mL})$. The product was extracted from the aqueous layer with AcOEt $(3 \times 10 \mathrm{~mL})$. The organic layer was dried over $\mathrm{Na}_{2} \mathrm{SO}_{4}$, concentrated and purified via flash chromatography to yield epi-clusianone (1) $(11.5 \mathrm{mg}, 46 \%)$ as a white powder. $R_{f}=0.5$ (petroleum ether/ethyl acetate, 10:1). HRESIMS calcd for $\mathrm{C}_{33} \mathrm{H}_{43} \mathrm{O}_{4}[\mathrm{M}+$ $\mathrm{H}]^{+}$503.3156, found 503.3150.<smiles>CC(=O)Oc1ccc(C(=O)Cl)cc1OC(C)=O</smiles><smiles>CC(C)=CC[C@H]1CC2(CC=C(C)C)C(=O)CC(=O)C1(CC=C(C)C)C2(C)C</smiles>

$15 a$
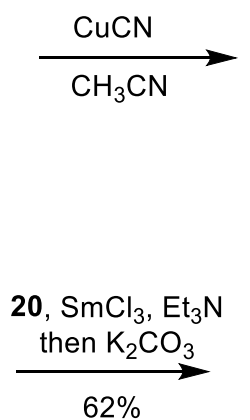

$62 \%$<smiles>CC(=O)Oc1ccc(C(=O)C#N)cc1OC(C)=O</smiles>

20<smiles>CC(C)=CC[C@H]1C[C@@]2(CC=C(C)C)C(=O)C1(CC=C(C)C)C(C)(C)[C@H]2C(=O)c1ccc(O)c(O)c1</smiles>

garcimultiflorone H (2)

A modified procedure based on Sen's method was used. ${ }^{2}$ Compound 20 was prepared as follows: To a mixture of $\mathrm{CuCN}(4.45 \mathrm{~g}, 50 \mathrm{mmol})$ in $\mathrm{CH}_{3} \mathrm{CN}(80 \mathrm{~mL})$ was added 3,4-bis(acetyloxy)benzoyl chloride (6.4 g, $\left.25 \mathrm{mmol}\right)$. The reaction mixture was refluxed for $4 \mathrm{~h}$. The mixture was then cooled, filtered and concentrated. The compound $\mathbf{2 0}$ was obtained by recrystallization from the residue in $\mathrm{CH}_{2} \mathrm{Cl}_{2}$ as a white solid $(4.9 \mathrm{~g}, 80 \%)$. ${ }^{1} \mathrm{H} \mathrm{NMR}\left(400 \mathrm{MHz}, \mathrm{CDCl}_{3}\right) \delta 8.08$ (dd, $J=8.6,2.2 \mathrm{~Hz}, 1 \mathrm{H}), 7.97(\mathrm{~d}, J=2.2 \mathrm{~Hz}, 1 \mathrm{H}), 7.48$ (d, $J=8.6 \mathrm{~Hz}, 1 \mathrm{H}), 2.34$ (d, $J=0.8 \mathrm{~Hz}, 6 \mathrm{H})$.

A solution of samarium (III) chloride $(2 \mathrm{mg}, 0.008 \mathrm{mmol})$, triethylamine $(15 \mu \mathrm{L}, 0.105 \mathrm{mmol})$ and compound $15 \mathbf{a}(35$ $\mathrm{mg}, 0.088 \mathrm{mmol})$ in $5 \mathrm{~mL}$ of toluene under $\mathrm{N}_{2}$ was stirred at $0{ }^{\circ} \mathrm{C}$ for $10 \mathrm{~min}$. After 20 (43 $\left.\mathrm{mg}, 0.176 \mathrm{mmol}\right)$ was added, the mixture was warmed up to room temperature and stirred for 2 hours. Then toluene was evaporated and potassium carbonate $(49 \mathrm{mg}, 0.352 \mathrm{mmol})$ and methanol $(5 \mathrm{~mL})$ were added into the resulting solution and stirred for 2 hours. The reaction was quenched with $10 \mathrm{~mL}$ of $1 \mathrm{~N} \mathrm{HCl}$. The product was extracted from the aqueous layer with AcOEt $(3 \times 20$ $\mathrm{mL}$ ). The organic layer was dried over $\mathrm{Na}_{2} \mathrm{SO}_{4}$, concentrated and purified via pre-HPLC to yield garcimultiflorone $\mathrm{H}(2)$ (29 mg, $62 \%$ ) as a light yellow gum. $R_{f}=0.6$ (dichloromethane/methanol, 10:1). HRESIMS calcd for $\mathrm{C}_{33} \mathrm{H}_{43} \mathrm{O}_{6}[\mathrm{M}+\mathrm{H}]^{+}$ 535.3054, found 535.3134. 


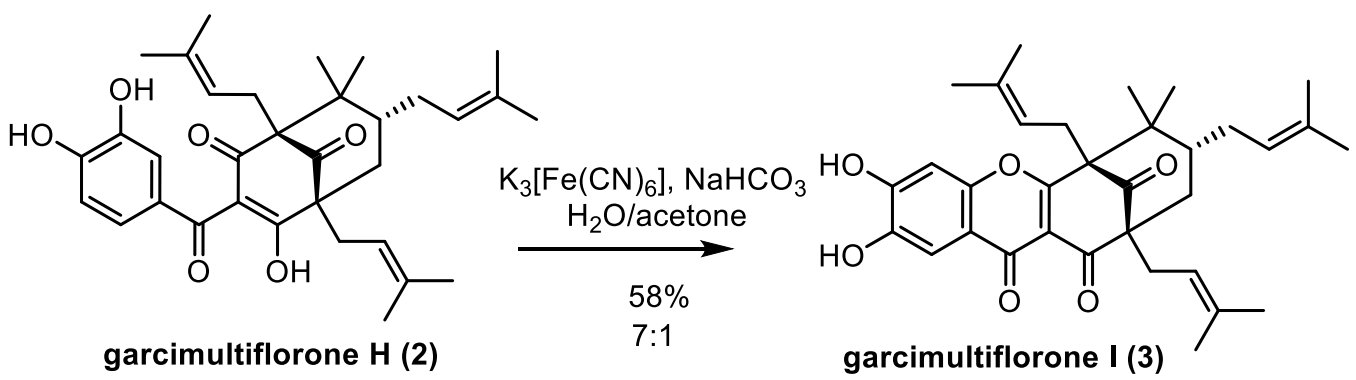

Garcimultiferone H (2) (30 mg, $0.056 \mathrm{mmol})$ and potassium ferricyanide (185 mg, $0.56 \mathrm{mmol})$ were dissolved in 2 $\mathrm{mL}$ of acetone under $\mathrm{N}_{2}$ at $0{ }^{\circ} \mathrm{C}$. Sodium bicarbonate $(47 \mathrm{mg}, 0.56 \mathrm{mmol})$ in $2 \mathrm{~mL}$ of $\mathrm{H}_{2} \mathrm{O}$ was added dropwise over one minute. Then the solution was stirred at $0{ }^{\circ} \mathrm{C}$ for 20 min and quenched with $1 \mathrm{~N} \mathrm{HCl}$ and then extracted with EtOAc $(3 \times$ $10 \mathrm{~mL}$ ). The combined organic layers are dried over $\mathrm{Na}_{2} \mathrm{SO}_{4}$, concentrated and purified via pre-HPLC to yield garcimultiflorone I (3) (17 mg, $58 \%$ ) as a light yellow gum. $R_{f}=0.5$ (dichloromethane/methanol, 10:1). HRESIMS calcd for $\mathrm{C}_{33} \mathrm{H}_{41} \mathrm{O}_{6}[\mathrm{M}+\mathrm{H}]^{+}$533.2898, found 533.2903.

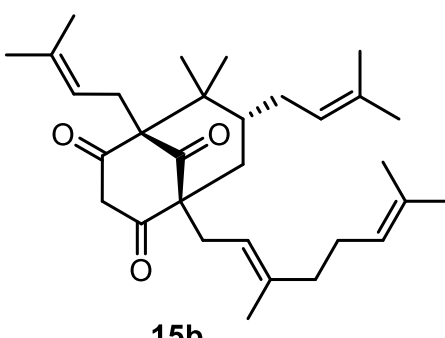

$15 b$

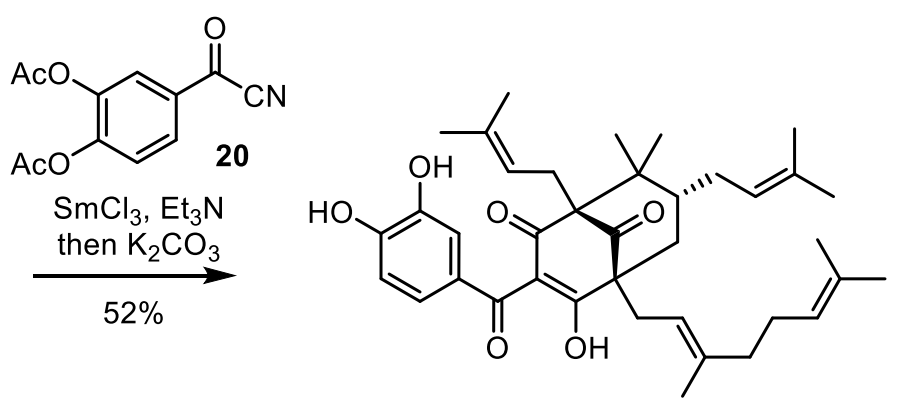

guttiferone I (4)

Prepared according to the general procedure that used for garcimultiflorone $\mathrm{H}$ with compound $\mathbf{1 5 b}(46.6 \mathrm{mg}, 0.1$ mmol), samarium (III) chloride $(2.5 \mathrm{mg}, 0.01 \mathrm{mmol})$, triethylamine (17 $\mu \mathrm{L}, 0.12 \mathrm{mmol})$ and 20 (49.5 mg, $0.2 \mathrm{mmol}) \mathrm{in} 5$ $\mathrm{mL}$ of toluene. The crude mixture was then treated by potassium carbonate $(56 \mathrm{mg}, 0.4 \mathrm{mmol})$ in $10 \mathrm{~mL}$ of methanol. The residue was purified via pre-HPLC to yield guttiferone I (4) (31 mg, $52 \%$ ) as a light yellow gum. $R_{f}=0.7$ $\left(\mathrm{Cl}_{2} \mathrm{CH}_{2} /\right.$ methanol, 10:1). HRESIMS calcd for $\mathrm{C}_{38} \mathrm{H}_{49} \mathrm{O}_{6}[\mathrm{M}-\mathrm{H}]^{-} 601.3529$, found 601.3553 .
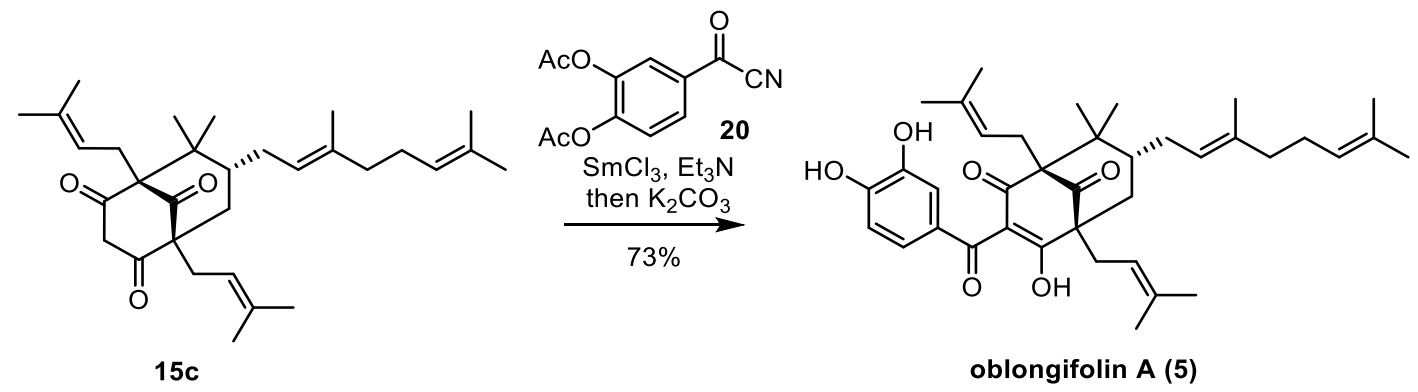

Prepared according to the general procedure that used for garcimultiflorone $\mathrm{H}$ with compound $\mathbf{1 5 c}(37 \mathrm{mg}, 0.08$ mmol), samarium (III) chloride ( $2 \mathrm{mg}, 0.008 \mathrm{mmol})$, triethylamine (13 $\mu \mathrm{L}, 0.095 \mathrm{mmol})$ and 20 (40 mg, $0.16 \mathrm{mmol}) \mathrm{in} 5$ $\mathrm{mL}$ of toluene. The crude mixture was then treated by potassium carbonate (44 $\mathrm{mg}, 0.32 \mathrm{mmol})$ in $10 \mathrm{~mL}$ of methanol. The residue was purified via pre-HPLC to yield oblongifolin A (5) (35 mg, $73 \%$ ) as a light yellow gum. $R_{f}=0.7$ (dichloromethane/methanol, 10:1). HRESIMS calcd for $\mathrm{C}_{38} \mathrm{H}_{51} \mathrm{O}_{6}[\mathrm{M}+\mathrm{H}]^{+}$603.3680, found 603.3688. 

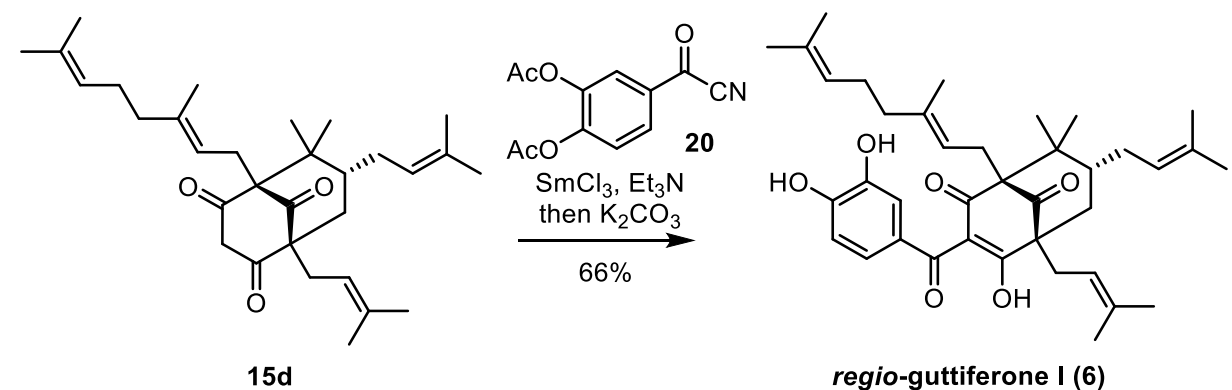

Prepared according to the general procedure that used for garcimultiflorone $\mathrm{H}$ with compound $\mathbf{1 5 d}$ (45 $\mathrm{mg}, 0.096$ mmol), samarium (III) chloride ( $2.5 \mathrm{mg}, 0.0096 \mathrm{mmol})$, triethylamine ( $16 \mu \mathrm{L}, 0.116 \mathrm{mmol})$ and 20 (47 mg, $0.192 \mathrm{mmol})$ in $5 \mathrm{~mL}$ of toluene. The crude mixture was then treated by potassium carbonate $(53 \mathrm{mg}, 0.384 \mathrm{mmol})$ in $10 \mathrm{~mL}$ of methanol. The residue was purified via pre-HPLC to yield regio-guttiferone I (6) (38 mg, 66 \%) as a light yellow gum. $R_{f}$ $=0.7$ (dichloromethane/methanol, 10:1). ${ }^{1} \mathrm{H}$ NMR $\left(600 \mathrm{MHz}, \mathrm{CD}_{3} \mathrm{OD}+0.1 \%\right.$ TFA- $d$ ) $\delta 7.18(\mathrm{~d}, J=2.1 \mathrm{~Hz}, 1 \mathrm{H}), 6.96(\mathrm{dd}$, $J=8.3,2.2 \mathrm{~Hz}, 1 \mathrm{H}), 6.67(\mathrm{~d}, J=8.3 \mathrm{~Hz}, 1 \mathrm{H}), 5.19(\mathrm{t}, J=7.3 \mathrm{~Hz}, 1 \mathrm{H}), 5.01-4.96(\mathrm{~m}, 1 \mathrm{H}), 4.96-4.96(\mathrm{~m}, 2 \mathrm{H}), 2.73(\mathrm{dd}$, $J=13.5,8.8 \mathrm{~Hz}, 1 \mathrm{H}), 2.63(\mathrm{dd}, J=13.3,4.4 \mathrm{~Hz}, 1 \mathrm{H}), 2.52(\mathrm{dd}, J=14.0,8.6 \mathrm{~Hz}, 1 \mathrm{H}), 2.44(\mathrm{dd}, J=14.0,4.8 \mathrm{~Hz}, 1 \mathrm{H})$, $2.20-2.12(\mathrm{~m}, 2 \mathrm{H}), 2.11-1.98(\mathrm{~m}, 2 \mathrm{H}), 1.97-1.85(\mathrm{~m}, 4 \mathrm{H}), 1.72(\mathrm{~s}, 3 \mathrm{H}), 1.69(\mathrm{~s}, 3 \mathrm{H}), 1.68(\mathrm{~s}, 3 \mathrm{H}), 1.64(\mathrm{~s}, 3 \mathrm{H}), 1.53$ (s, 3H), $1.52-1.49(\mathrm{~m}, 1 \mathrm{H}), 1.47(\mathrm{~s}, 3 \mathrm{H}), 1.42(\mathrm{~s}, 3 \mathrm{H}), 1.27(\mathrm{~s}, 3 \mathrm{H}), 1.03(\mathrm{~s}, 3 \mathrm{H}) \cdot{ }^{13} \mathrm{C}$ NMR $\left(151 \mathrm{MHz}, \mathrm{CD}_{3} \mathrm{OD}+0.1 \%\right.$ TFA-d) $\delta 209.8,195.8,195.4,195.0,152.6,146.2,139.5,135.6,133.8,132.2,129.5,125.4,125.3,125.1,120.8,120.3$, $117.9,117.3,115.1,67.8,62.3,49.3,47.8,41.0,32.2,30.8,30.1,27.9,27.3,26.8,26.3,26.0,25.8,23.3,18.2,18.1,17.7$, 17.0. HRESIMS calcd for $\mathrm{C}_{38} \mathrm{H}_{51} \mathrm{O}_{6}[\mathrm{M}+\mathrm{H}]^{+}$603.3680, found 603.3707 .

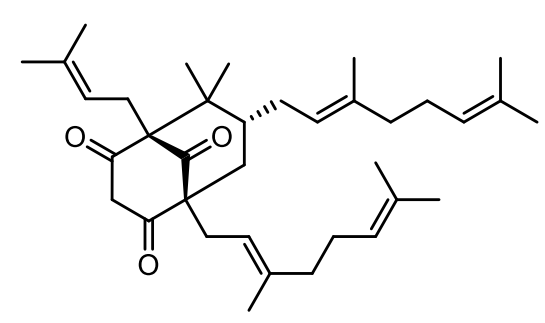

$15 e$
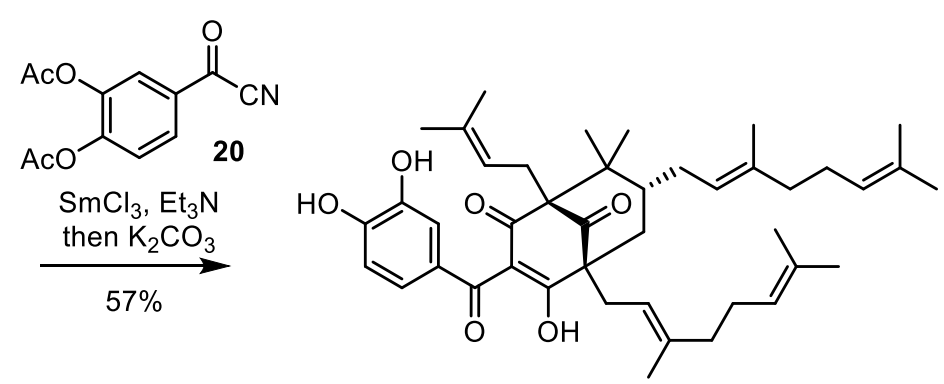

oblongifolin D (7)

Prepared according to the general procedure that used for garcimultiflorone $\mathrm{H}$ with compound 15e $(32 \mathrm{mg}, 0.06$ mmol), samarium (III) chloride ( $1.5 \mathrm{mg}, 0.006 \mathrm{mmol})$, triethylamine (10 $\mu \mathrm{L}, 0.071 \mathrm{mmol})$ and 20 (30 mg, $0.12 \mathrm{mmol})$ in $5 \mathrm{~mL}$ of toluene. The crude mixture was then treated by potassium carbonate $(33 \mathrm{mg}, 0.24 \mathrm{mmol})$ in $10 \mathrm{~mL}$ of methanol. The residue was purified via pre-HPLC to yield oblongifolin D (7) $(23 \mathrm{mg}, 57 \%)$ as a light yellow gum. $R_{f}=0.8$ (dichloromethane/methanol, 10:1). HRESIMS calcd for $\mathrm{C}_{43} \mathrm{H}_{59} \mathrm{O}_{6}[\mathrm{M}+\mathrm{H}]^{+}$671.4306, found 671.4326. 

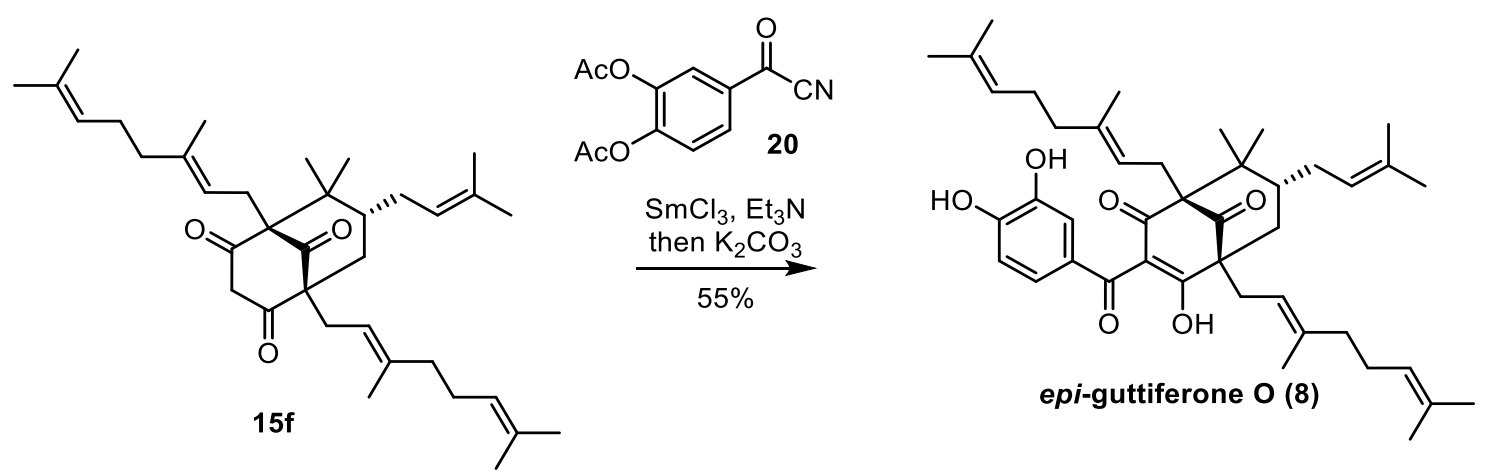

Prepared according to the general procedure that used for garcimultiflorone $\mathrm{H}$ with compound $\mathbf{1 5 f}(32 \mathrm{mg}, 0.06$ mmol), samarium (III) chloride $(1.5 \mathrm{mg}, 0.006 \mathrm{mmol})$, triethylamine $(10 \mu \mathrm{L}, 0.071 \mathrm{mmol})$ and $20(30 \mathrm{mg}, 0.12 \mathrm{mmol}) \mathrm{in}$ $5 \mathrm{~mL}$ of toluene. The crude mixture was then treated by potassium carbonate $(33 \mathrm{mg}, 0.24 \mathrm{mmol})$ in $10 \mathrm{~mL}$ of methanol. The residue was purified via pre-HPLC to yield epi-guttiferone $\mathrm{O}(\mathbf{8})(21.8 \mathrm{mg}, 55 \%)$ as a light yellow gum. $R_{f}=0.8$ (dichloromethane/methanol, 10:1). ${ }^{1} \mathrm{H}$ NMR $\left(400 \mathrm{MHz}, \mathrm{CD}_{3} \mathrm{OD}+0.1 \%\right.$ TFA- $d$ ) $\delta 7.20(\mathrm{~d}, J=2.2 \mathrm{~Hz}, 1 \mathrm{H}), 6.96(\mathrm{dd}, J=$ $8.3,2.3 \mathrm{~Hz}, 1 \mathrm{H}), 6.65(\mathrm{~d}, J=8.4 \mathrm{~Hz}, 1 \mathrm{H}), 5.19(\mathrm{t}, J=7.3 \mathrm{~Hz}, 1 \mathrm{H}), 5.07$ (t, $J=7.6 \mathrm{~Hz}, 1 \mathrm{H}), 5.02-4.92(\mathrm{~m}, 2 \mathrm{H}), 4.91-$ $4.89(\mathrm{~m}, 1 \mathrm{H}), 2.74(\mathrm{dd}, J=13.5,8.7 \mathrm{~Hz}, 1 \mathrm{H}), 2.62(\mathrm{dd}, J=13.8,5.2 \mathrm{~Hz}, 1 \mathrm{H}), 2.58-2.43(\mathrm{~m}, 2 \mathrm{H}), 2.22-2.16(\mathrm{~m}, 2 \mathrm{H})$, $2.12-2.05(\mathrm{~m}, 2 \mathrm{H}), 2.05-1.84(\mathrm{~m}, 8 \mathrm{H}), 1.69(\mathrm{~s}, 6 \mathrm{H}), 1.64(\mathrm{~s}, 3 \mathrm{H}), 1.61(\mathrm{~s}, 3 \mathrm{H}), 1.59-1.50(\mathrm{~m}, 1 \mathrm{H}), 1.54(\mathrm{~s}, 6 \mathrm{H}), 1.47$ $(\mathrm{s}, 3 \mathrm{H}), 1.43(\mathrm{~s}, 3 \mathrm{H}), 1.25(\mathrm{~s}, 3 \mathrm{H}) 1.03(\mathrm{~s}, 3 \mathrm{H}) .{ }^{13} \mathrm{C} \mathrm{NMR}\left(101 \mathrm{MHz}, \mathrm{CD}_{3} \mathrm{OD}+0.1 \% \mathrm{TFA}-d\right) \delta 209.8,195.9,195.4,195.1$, $152.6,146.2,139.5,139.2,133.8,132.21,132.19,129.5,125.4,125.3,125.2,120.5,120.4,117.9,117.3,115.1,67.7$, $62.3,49.2,47.8,41.1,40.9,32.0,30.1,27.9,27.8,27.3,26.8,25.98,25.96,25.8,23.3,18.1,17.82,17.74,16.96,16.9$. HRESIMS calcd for $\mathrm{C}_{43} \mathrm{H}_{57} \mathrm{O}_{6}[\mathrm{M}-\mathrm{H}]^{-} 669.4160$, found 669.4177 .
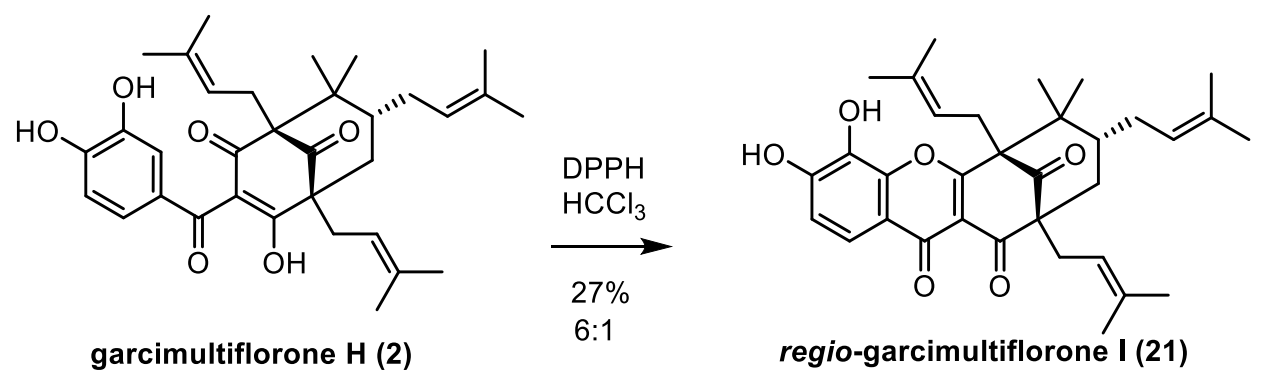

Garcimultiferone H (2) (29 mg, $0.054 \mathrm{mmol}$ ) was treated with 2,2-di(4-tert-octylphenyl)-1-picrylhydrazyl (DPPH) $(70.2 \mathrm{mg}, 0.11 \mathrm{mmol})$ in $\mathrm{CHCl}_{3}$ at room temperature under darkness for $24 \mathrm{~h}$. After evaporation of the solvent in vacuo, the residue was applied to a silica gel column to give $7.7 \mathrm{mg}$ of regio-garcimultiflorone I (21) as a pale yellow powder $(27 \%) . R_{f}=0.4$ (dichloromethane/methanol, 10:1). ${ }^{1} \mathrm{H}$ NMR (600 MHz, DMSO-d $) \delta 10.66(\mathrm{~s}, 1 \mathrm{H}), 9.52(\mathrm{~s}, 1 \mathrm{H}), 7.38(\mathrm{~d}$, $J=8.6 \mathrm{~Hz}, 1 \mathrm{H}), 6.98(\mathrm{~d}, J=8.6 \mathrm{~Hz}, 1 \mathrm{H}), 5.18(\mathrm{t}, J=7.6 \mathrm{~Hz}, 1 \mathrm{H}), 4.85-4.79(\mathrm{~m}, 1 \mathrm{H}), 4.52-4.45(\mathrm{~m}, 1 \mathrm{H}), 2.89(\mathrm{dd}, J=$ $13.9,8.8 \mathrm{~Hz}, 1 \mathrm{H}), 2.71(\mathrm{dd}, J=14.0,4.9 \mathrm{~Hz}, 1 \mathrm{H}), 2.40(\mathrm{dd}, J=14.4,8.2 \mathrm{~Hz}, 1 \mathrm{H}), 2.33(\mathrm{dd}, J=14.5,6.9 \mathrm{~Hz}, 1 \mathrm{H}), 2.26$ $(\mathrm{dd}, J=14.4,10.7 \mathrm{~Hz}, 1 \mathrm{H}), 1.93(\mathrm{~d}, J=14.5 \mathrm{~Hz}, 1 \mathrm{H}), 1.80(\mathrm{dd}, J=14.4,4.3 \mathrm{~Hz}, 1 \mathrm{H}), 1.72-1.64(\mathrm{~m}, 2 \mathrm{H}), 1.67(\mathrm{~s}, 3 \mathrm{H})$, $1.61(\mathrm{~s}, 3 \mathrm{H}), 1.601(\mathrm{~s}, 3 \mathrm{H}), 1.596(\mathrm{~s}, 3 \mathrm{H}), 1.41(\mathrm{~s}, 3 \mathrm{H}), 1.35(\mathrm{~s}, 3 \mathrm{H}), 1.20(\mathrm{~s}, 3 \mathrm{H}), 1.03(\mathrm{~s}, 3 \mathrm{H}) .{ }^{13} \mathrm{C} \mathrm{NMR}(101 \mathrm{MHz}$, DMSO- $\left.d_{6}\right) \delta 205.8,190.7,176.9,172.1,150.7,144.4,134.4,133.7,133.6,132.4,122.9,119.5,118.3,117.2,116.3,115.5$, $114.5,65.9,62.1,47.4,45.9,37.0,28.4,27.8,26.6,25.9,25.8,25.7,25.5,20.4,17.8,17.5$. HRESIMS calcd for $\mathrm{C}_{33} \mathrm{H}_{41} \mathrm{O}_{6}$ $[\mathrm{M}+\mathrm{H}]^{+}$533.2898, found 533.2906. 
Table S2. Comparison of ${ }^{1} \mathrm{H}$ NMR and ${ }^{13} \mathrm{C}$ NMR data for epi-clusianone $(\mathbf{1})^{4}$ in $\mathrm{CDCl}_{3}$

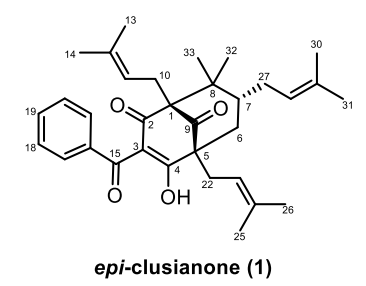

\begin{tabular}{|c|c|c|c|c|}
\hline \multirow[b]{2}{*}{ Position } & \multicolumn{2}{|c|}{ epi-clusianone (a) isolated } & \multicolumn{2}{|c|}{ epi-clusianone (a) synthetic } \\
\hline & $\delta \mathrm{C}(151 \mathrm{MHz})$ & $\delta \mathrm{H}\left(600 \mathrm{MH}_{\mathrm{Z}}\right)$ & $\delta \mathrm{C}\left(101 \mathrm{MH}_{\mathrm{Z}}\right)$ & $\delta \mathrm{H}\left(400 \mathrm{MH}_{\mathrm{z}}\right)$ \\
\hline 1 & 68.6 & & 68.8 & \\
\hline 2 & 193.7 & & 193.8 & \\
\hline 3 & 115.9 & & 116.0 & \\
\hline 4 & 196.6 & & 196.9 & \\
\hline 5 & 58.4 & & 58.6 & \\
\hline $6 a x$ & 39.0 & 2.22, dd $(14.4,7.0)$ & 39.2 & $2.22, \mathrm{~m}$ \\
\hline 6 eq & & $2.26, \mathrm{dd}(14.4,1.8)$ & & $2.26, \mathrm{~m}$ \\
\hline 7 & 46.3 & 1.44 , overlapped & 46.5 & 1.44 , overlapped \\
\hline 8 & 48.3 & & 48.5 & \\
\hline 9 & 208.1 & & 208.3 & \\
\hline 10 & 26.2 & $2.47, \mathrm{~m}$ & 26.2 & $2.46, \mathrm{~m}$ \\
\hline 11 & 120.1 & $5.12, \mathrm{~m}$ & 120.3 & $5.13, \mathrm{~m}$ \\
\hline 12 & 134.8 & & 135.0 & \\
\hline 13 & 18.1 & $1.70, \mathrm{~s}$ & 18.3 & $1.71, \mathrm{~s}$ \\
\hline 14 & 26.0 & $1.66, \mathrm{~s}$ & 25.9 & $1.67, \mathrm{~s}$ \\
\hline 15 & 197.5 & & 197.7 & \\
\hline 16 & 136.8 & & 136.9 & \\
\hline 17,21 & 129.0 & $7.52, \mathrm{~d}(7.6)$ & 129.1 & $7.52, \mathrm{~m}$ \\
\hline 18,20 & 127.8 & $7.38, \mathrm{t}(7.6)$ & 128.0 & $7.37, \mathrm{t}(7.6)$ \\
\hline 19 & 132.6 & $7.53, \mathrm{t}(7.6)$ & 132.8 & $7.53, \mathrm{~m}$ \\
\hline 22 & 30.5 & $2.62, \mathrm{~m}$ & 30.5 & $2.63, \mathrm{~m}$ \\
\hline 23 & 118.9 & $5.15, \mathrm{~m}$ & 119.1 & $5.13, \mathrm{~m}$ \\
\hline 24 & 134.7 & & 134.9 & \\
\hline 25 & 18.1 & $1.67, \mathrm{~s}$ & 18.3 & $1.67 \mathrm{~s}$ \\
\hline 26 & 26.0 & $1.80, \mathrm{~s}$ & 26.2 & $1.80, \mathrm{~s}$ \\
\hline 27 & 28.9 & 2.05 , overlapped & 29.1 & 2.05 , overlapped \\
\hline & & 2.16 , overlapped & & 2.16 , overlapped \\
\hline 28 & 123.5 & $4.85, \mathrm{~m}$ & 123.6 & $4.86, \mathrm{~m}$ \\
\hline 29 & 132.9 & & 133.1 & \\
\hline 30 & 17.7 & $1.43, \mathrm{~s}$ & 17.9 & $1.43, \mathrm{~s}$ \\
\hline 31 & 25.7 & $1.58, \mathrm{~s}$ & 25.9 & $1.58, \mathrm{~s}$ \\
\hline 32 & 27.0 & $0.97, \mathrm{~s}$ & 27.1 & $0.97, \mathrm{~s}$ \\
\hline 33 & 22.5 & $1.13, \mathrm{~s}$ & 22.7 & $1.14, \mathrm{~s}$ \\
\hline
\end{tabular}

epi-clusianone (b) isolated

$\begin{array}{lllll}\text { Position } & \delta \mathrm{C}(151 \mathrm{MHz}) & \delta \mathrm{H}\left(600 \mathrm{MH}_{\mathrm{Z}}\right) & \delta \mathrm{C}\left(101 \mathrm{MH}_{\mathrm{Z}}\right) & \delta \mathrm{H}(400 \mathrm{c} \\ 1 & 65.5 & & 196.9 & \\ 2 & 196.7 & & 115.7 & \\ 3 & 115.5 & & 193.8 & \\ 4 & 193.6 & & 63.4 & 2.01, \mathrm{~m} \\ 5 & 63.2 & 2.01, \mathrm{dd}(14.4,7.0) & 40.5 & 2.11, \mathrm{~m}\end{array}$




\begin{tabular}{|c|c|c|c|c|}
\hline 7 & 46.6 & 1.44 , overlapped & 46.7 & 1.44 , overlapped \\
\hline 8 & 48.8 & & 49.0 & \\
\hline 9 & 208.3 & & 208.4 & \\
\hline 10 & 25.9 & $2.72, \mathrm{~m}$ & 26.2 & $2.72, \mathrm{~m}$ \\
\hline 11 & 118.6 & $4.8, \mathrm{~m}$ & 118.7 & $4.8, \mathrm{~m}$ \\
\hline 12 & 134.8 & & 135.0 & \\
\hline 13 & 18.0 & $1.68, \mathrm{~s}$ & 18.2 & $1.67, \mathrm{~s}$ \\
\hline 14 & 25.8 & $1.61, \mathrm{~s}$ & 25.9 & $1.61, \mathrm{~s}$ \\
\hline 15 & 197.2 & & 197.4 & \\
\hline 16 & 136.8 & & 136.9 & \\
\hline 17,21 & 128.9 & $7.55, \mathrm{~d}(7.6)$ & 129.1 & $7.55, \mathrm{~m}$ \\
\hline 18,20 & 127.8 & $7.40, \mathrm{t}(7.6)$ & 128.0 & $7.37, \mathrm{t}(7.6)$ \\
\hline 19 & 132.6 & $7.53, \mathrm{t}(7.6)$ & 132.8 & $7.53, \mathrm{~m}$ \\
\hline \multirow[t]{2}{*}{22} & 30.7 & $2.44, \mathrm{~m}$ & 30.7 & $2.44, \mathrm{~m}$ \\
\hline & & $2.52, \mathrm{~m}$ & & $2.53, \mathrm{~m}$ \\
\hline 23 & 119.7 & $5.24, \mathrm{~m}$ & 119.8 & $5.22, \mathrm{~m}$ \\
\hline 24 & 134.6 & & 134.9 & \\
\hline 25 & 18.2 & $1.66, \mathrm{~s}$ & 18.3 & $1.67, \mathrm{~s}$ \\
\hline 26 & 26.1 & $1.76, \mathrm{~s}$ & 26.2 & $1.76, \mathrm{~s}$ \\
\hline 27 & 28.9 & 2.00, overlapped & 29.1 & 2.00 , overlapped \\
\hline 28 & 124.0 & $4.87, \mathrm{~m}$ & 124.2 & $4.86, \mathrm{~m}$ \\
\hline 29 & 133.0 & & 133.1 & \\
\hline 30 & 17.8 & $1.50, \mathrm{~s}$ & 17.9 & $1.50, \mathrm{~s}$ \\
\hline 31 & 25.7 & $1.64, \mathrm{~s}$ & 25.9 & $1.64, \mathrm{~s}$ \\
\hline 32 & 26.9 & $1.06, \mathrm{~s}$ & 26.7 & $1.06, \mathrm{~s}$ \\
\hline 33 & 22.8 & $1.30, \mathrm{~s}$ & 22.8 & $1.30, \mathrm{~s}$ \\
\hline
\end{tabular}

Table S3. Comparison of ${ }^{1} \mathrm{H}$ NMR and ${ }^{13} \mathrm{C}$ NMR data for natural and synthetic garcimultiflorone $\mathrm{H}(2)^{5}$ in $\mathrm{CD}_{3} \mathrm{OD}+0.1 \%$ TFA- $d$

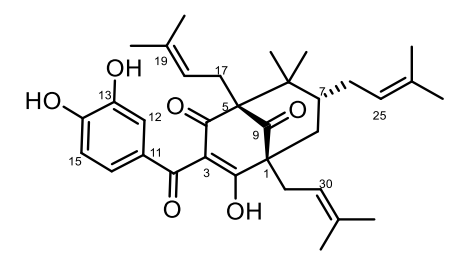

garcimultiflorone $H$ (2)

\begin{tabular}{|c|c|c|c|c|}
\hline \multirow[b]{2}{*}{ Position } & \multicolumn{2}{|c|}{ garcimultiflorone $\mathbf{H}$ isolated } & \multicolumn{2}{|c|}{ garcimultiflorone $\mathbf{H}$ synthetic } \\
\hline & $\delta \mathrm{C}\left(151 \mathrm{MH}_{\mathrm{Z}}\right)$ & $\delta \mathrm{H}\left(600 \mathrm{MH}_{\mathrm{Z}}\right)$ & $\delta \mathrm{C}\left(101 \mathrm{MHz}_{\mathrm{Z}}\right)$ & $\delta \mathrm{H}\left(600 \mathrm{MH}_{\mathrm{Z}}\right)$ \\
\hline 1 & 62.4 & & 62.2 & \\
\hline 2 & 195.2 & & 195.3 & \\
\hline 3 & 117.9 & & 117.8 & \\
\hline 4 & 194.7 & & 194.7 & \\
\hline 5 & 67.7 & & 67.8 & \\
\hline
\end{tabular}




\begin{tabular}{|c|c|c|c|c|}
\hline 6 & 49.3 & & $\mathrm{CD}_{3} \mathrm{OD}$ signal & \\
\hline 7 & 47.8 & $1.51, \mathrm{~m}$ & 47.8 & $1.51, \mathrm{~m}$ \\
\hline 8 eq & 40.8 & $2.15, \mathrm{~d}(13.7)$ & 40.8 & $2.15, \mathrm{~d}(14.1)$ \\
\hline $8 \mathrm{ax}$ & & $2.08, \mathrm{dd}(13.7,6.6)$ & & $2.08, \mathrm{dd}(13.8,7.4)$ \\
\hline 9 & 209.9 & & 209.9 & \\
\hline 10 & 196.0 & & 195.9 & \\
\hline 11 & 129.7 & & 129.5 & \\
\hline 12 & 117.4 & $7.17, \mathrm{~s}$ & 117.4 & $7.17, \mathrm{~d}(2.1)$ \\
\hline 13 & 146.2 & & 146.2 & \\
\hline 14 & 152.6 & & 152.5 & \\
\hline 15 & 115.2 & $6.69, \mathrm{~d}(8.2)$ & 115.2 & $6.70, \mathrm{~d}(8.3)$ \\
\hline 16 & 125.1 & $6.97, \mathrm{~d}(8.2)$ & 125.1 & $6.97, \mathrm{dd}(8.3,2.1)$ \\
\hline \multirow[t]{2}{*}{17} & 27.1 & $2.72, \mathrm{t}$-like & 27.1 & $2.72, \mathrm{dd}(13.6,9.0)$ \\
\hline & & 2.58, d-like & & 2.58, d-like \\
\hline 18 & 120.8 & 4.93, br s & 120.8 & 4.93 , br s \\
\hline 19 & 135.6 & & 135.7 & \\
\hline 20 & 26.3 & $1.71, \mathrm{~s}$ & 26.3 & $1.72, \mathrm{~s}$ \\
\hline 21 & 18.2 & $1.68, \mathrm{~s}$ & 18.2 & $1.68, \mathrm{~s}$ \\
\hline 22 & 27.3 & $1.01, \mathrm{~s}$ & 27.3 & $1.02, \mathrm{~s}$ \\
\hline 23 & 23.2 & $1.24, \mathrm{~s}$ & 23.2 & $1.25, \mathrm{~s}$ \\
\hline \multirow[t]{2}{*}{24} & 30.2 & 2.15, d-like & 30.1 & 2.16, d-like \\
\hline & & $2.06, \mathrm{~m}$ & & $2.05, \mathrm{~m}$ \\
\hline 25 & 125.4 & 4.87 , overlap & 125.4 & 4.87 , overlap \\
\hline 26 & 133.7 & & 133.7 & \\
\hline 27 & 26.0 & $1.67, \mathrm{~s}$ & 26.0 & $1.65, \mathrm{~s}$ \\
\hline 28 & 18.1 & $1.50, \mathrm{~s}$ & 18.1 & $1.48, \mathrm{~s}$ \\
\hline \multirow[t]{2}{*}{29} & 32.1 & $2.50, \mathrm{t}$-like & 32.1 & $2.52, \mathrm{dd}(14.0,8.3)$ \\
\hline & & 2.47, d-like & & 2.47, d-like \\
\hline 30 & 120.9 & 5.16, br s & 120.8 & $5.17, \mathrm{t}(7.2)$ \\
\hline 31 & 135.5 & & 135.5 & \\
\hline 32 & 18.3 & $1.68, \mathrm{~s}$ & 18.3 & \\
\hline 33 & 26.3 & $1.71, \mathrm{~s}$ & 26.3 & \\
\hline
\end{tabular}

Table S4. Comparison of ${ }^{1} \mathrm{H}$ NMR and ${ }^{13} \mathrm{C}$ NMR data for natural and synthetic garcimultiflorone I (3) $)^{6}$ in DMSO- $d_{6}$

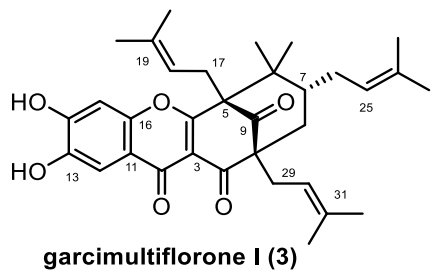

\begin{tabular}{|c|c|c|c|c|}
\hline \multirow[b]{2}{*}{ Position } & \multicolumn{2}{|c|}{ garcimultiflorone I isolated } & \multicolumn{2}{|c|}{ garcimultiflorone I synthetic } \\
\hline & $\delta \mathrm{C}\left(151 \mathrm{MH}_{\mathrm{Z}}\right)$ & $\delta \mathrm{H}\left(400 \mathrm{MH}_{\mathrm{Z}}\right)$ & $\delta \mathrm{C}\left(101 \mathrm{MH}_{\mathrm{Z}}\right)$ & $\delta \mathrm{H}\left(400 \mathrm{MH}_{\mathrm{Z}}\right)$ \\
\hline 1 & 65.7 & & 65.8 & \\
\hline
\end{tabular}


116.1

$7.23, \mathrm{~s}$

108.1

146.0

145.6

153.1

$6.87, \mathrm{~s}$

103.1

$1.81, \mathrm{dd}(14.3,4.5)$

28.5

17.5

Several ${ }^{1} \mathrm{H}$ NMR and ${ }^{13} \mathrm{C}$ NMR data in red are not consistent. After comparing the data with the original NMR file, we found this inconsistence is due to the poor quality of the original spectrum.

Table S5. Comparison of ${ }^{1} \mathrm{H}$ NMR and ${ }^{13} \mathrm{C}$ NMR data for natural and synthetic guttiferone $\mathrm{I}(4)^{6}$ in $\mathrm{CD}_{3} \mathrm{OD}+0.1 \%$ TFA- $d$ 


\begin{tabular}{|c|c|c|c|c|}
\hline & guttiferone I isolated & & guttiferone I synthetic & \\
\hline Position & $\delta \mathrm{C}\left(125 \mathrm{MH}_{\mathrm{Z}}\right)$ & $\delta \mathrm{H}\left(500 \mathrm{MH}_{\mathrm{Z}}\right)$ & $\delta \mathrm{C}\left(101 \mathrm{MH}_{\mathrm{Z}}\right)$ & $\delta \mathrm{H}\left(400 \mathrm{MH}_{\mathrm{Z}}\right)$ \\
\hline 1 & 194.9 & & 194.9 & \\
\hline 2 & 118.4 & & 117.8 & \\
\hline 3 & 195.4 & & 195.4 & \\
\hline 4 & 68.1 & & 68.0 & \\
\hline 5 & 47.9 & & 47.8 & \\
\hline 6 & 41.0 & $1.98, \mathrm{~m}$ & 41.0 & $1.98, \mathrm{~m}$ \\
\hline 7 & 40.7 & $2.04, \mathrm{~m}$ & 40.7 & $2.09, \mathrm{~m}$ \\
\hline 8 & 61.8 & & 61.7 & \\
\hline 9 & 209.8 & & 209.8 & \\
\hline 10 & 196.0 & & 195.9 & \\
\hline 11 & 129.6 & & 129.5 & \\
\hline 12 & 117.3 & $7.19, \mathrm{~d}(1.9)$ & 117.3 & $7.19, \mathrm{~d}(2.1)$ \\
\hline 13 & 146.2 & & 146.2 & \\
\hline 14 & 152.5 & & 152.5 & \\
\hline 15 & 115.1 & $6.68, \mathrm{~d}(8.3)$ & 115.1 & $6.68, \mathrm{~d}(8.3)$ \\
\hline 16 & 125.2 & 6.98, dd $(1.9,8.3)$ & 125.2 & $6.97, \mathrm{dd}(2.2,8.3)$ \\
\hline \multirow[t]{2}{*}{17} & 27.1 & $2.54, \mathrm{~m}$ & 27.1 & $2.57, \mathrm{~m}$ \\
\hline & & 2.75, br dd $(9.1,13.5)$ & & 2.73, br dd $(9.0,13.5)$ \\
\hline 18 & 120.8 & 4.90, br t $(9.1)$ & 120.8 & $4.95, \mathrm{~m}$ \\
\hline 19 & 135.7 & & 135.7 & \\
\hline 20 & 25.9 & $1.65, \mathrm{~s}$ & 26.0 & $1.65, \mathrm{~s}$ \\
\hline 21 & 18.2 & $1.68, \mathrm{~s}$ & 18.3 & $1.68, \mathrm{~s}$ \\
\hline 22 & 23.2 & $1.24, \mathrm{~s}$ & 23.2 & $1.24, \mathrm{~s}$ \\
\hline 23 & 27.3 & $1.02, \mathrm{~s}$ & 27.3 & $1.02, \mathrm{~s}$ \\
\hline 24 & 30.1 & $2.09, \mathrm{~m}$ & 30.1 & $2.13, \mathrm{~m}$ \\
\hline 25 & 125.4 & $5.05, \mathrm{~m}$ & 125.4 & $4.98, \mathrm{~m}$ \\
\hline 26 & 133.7 & & 133.7 & \\
\hline 27 & 26.3 & $1.65, \mathrm{~s}$ & 26.4 & $1.65, \mathrm{~s}$ \\
\hline 28 & 18.1 & $1.48, \mathrm{~s}$ & 18.1 & $1.48, \mathrm{~s}$ \\
\hline 29 & 31.8 & $2.52, \mathrm{~m}$ & 31.8 & $2.51, \mathrm{~m}$ \\
\hline 30 & 120.5 & $5.17, \mathrm{~m}$ & 120.5 & $5.17, \mathrm{t}(7.5)$ \\
\hline 31 & 139.2 & & 139.1 & \\
\hline 32 & 27.7 & $2.00, \mathrm{~m}$ & 27.7 & $2.02, \mathrm{~m}$ \\
\hline 33 & 27.8 & $1.99, \mathrm{~m}$ & 27.7 & $2.00, \mathrm{~m}$ \\
\hline 34 & 125.1 & $5.09, \mathrm{~m}$ & 125.2 & $5.07, \mathrm{~m}$ \\
\hline
\end{tabular}




$\begin{array}{lllll}35 & 132.2 & & 132.2 & \\ 36 & 25.9 & 1.58, \mathrm{~s} & 25.9 & 1.58, \mathrm{~s} \\ 37 & 17.8 & 1.53, \mathrm{~s} & 17.8 & 1.53, \mathrm{~s} \\ 38 & 16.8 & 1.69, \mathrm{~s} & 16.9 & 1.69, \mathrm{~s}\end{array}$

Table S6. Comparison of ${ }^{1} \mathrm{H}$ NMR and ${ }^{13} \mathrm{C}$ NMR data for natural and synthetic oblongifolin $\mathrm{A}(\mathbf{5}){ }^{7}$ in $\mathrm{CD}_{3} \mathrm{OD}+0.1 \%$ TFA- $d$

\begin{tabular}{|c|c|c|c|c|c|c|}
\hline \multirow[b]{2}{*}{ Position } & \multicolumn{2}{|c|}{ oblongifolin A isolated } & \multicolumn{2}{|c|}{ oblongifolin A synthetic } & \multicolumn{2}{|c|}{ oblongifolin A synthetic ${ }^{8}$} \\
\hline & $\delta \mathrm{C}(151 \mathrm{MHz})$ & $\delta \mathrm{H}\left(600 \mathrm{MH}_{\mathrm{z}}\right)$ & $\delta \mathrm{C}\left(101 \mathrm{MH}_{\mathrm{Z}}\right)$ & $\delta \mathrm{H}\left(400 \mathrm{MH}_{\mathrm{z}}\right)$ & $\delta \mathrm{C}\left(125 \mathrm{MH}_{\mathrm{Z}}\right)$ & $\delta \mathrm{H}\left(500 \mathrm{MH}_{\mathrm{Z}}\right)$ \\
\hline 1 & 196.3 & & 195.9 & & 196.0 & \\
\hline 2 & 117.9 & & 117.9 & & 117.9 & \\
\hline 3 & 195.9 & & 195.3 & & 195.2 & \\
\hline 4 & 68.8 & & 68.0 & & 68.2 & \\
\hline 5 & 48.4 & & $\mathrm{CD}_{3} \mathrm{OD}$ signal & & $\mathrm{CD}_{3} \mathrm{OD}$ signal & \\
\hline 6 & 47.8 & $1.53, \mathrm{~m}$ & 47.8 & $1.53, \mathrm{~m}$ & 47.8 & $1.52, \mathrm{~m}$ \\
\hline 7 & 40.8 & $\begin{array}{l}\text { eq. } 2.16, \mathrm{~m} \\
\text { ax. } 2.08, \mathrm{~m}\end{array}$ & 40.9 & $\begin{array}{l}\text { eq. } 2.18, \mathrm{~m} \\
\text { ax. } 2.09, \mathrm{~m}\end{array}$ & 40.8 & $\begin{array}{l}2.21-2.12, \mathrm{~m} \\
2.10-2.02, \mathrm{~m}\end{array}$ \\
\hline 8 & 61.8 & & 61.9 & & 61.6 & \\
\hline 9 & 209.8 & & 209.9 & & 210.0 & \\
\hline 10 & 195.8 & & 194.7 & & 194.8 & \\
\hline 11 & 129.5 & & 129.5 & & 129.6 & \\
\hline 12 & 117.4 & $7.17, \mathrm{~d}(2.1)$ & 117.4 & $7.18, \mathrm{~d}(2.2)$ & 117.4 & $7.18, \mathrm{~d}(2.2)$ \\
\hline 13 & 146.2 & & 146.2 & & 146.2 & \\
\hline 14 & 152.5 & & 152.6 & & 152.5 & \\
\hline 15 & 115.2 & $6.70, \mathrm{~d}(8.3)$ & 115.2 & $6.70, \mathrm{~d}(8.3)$ & 115.1 & $6.70, \mathrm{~d}(8.3)$ \\
\hline 16 & 125.1 & $\begin{array}{l}6.97, \mathrm{dd}(8.3, \\
2.1)\end{array}$ & 125.1 & $\begin{array}{l}6.98, \text { dd }(8.4, \\
2.2)\end{array}$ & 125.1 & $\begin{array}{l}6.98, \mathrm{dd} \quad(8.3, \\
2.2)\end{array}$ \\
\hline 17 & 27.0 & $\begin{array}{l}2.71, \mathrm{dd} \quad(9.0 \\
13.0) \\
2.58, \mathrm{~m}\end{array}$ & 27.1 & $\begin{array}{l}2.73, \text { dd (9.1, } \\
13.6) \\
2.58, \mathrm{~m}\end{array}$ & 27.0 & $\begin{array}{l}2.72, \mathrm{dd} \quad(9.0, \\
13.1) \\
2.58, \mathrm{~m}\end{array}$ \\
\hline 18 & 120.7 & $4.94, \mathrm{~m}$ & 120.8 & $4.95, \mathrm{~m}$ & 120.8 & $4.95, \mathrm{~m}$ \\
\hline 19 & 135.5 & & 135.5 & & 135.4 & \\
\hline 20 & 26.3 & $1.71, \mathrm{~s}$ & 26.3 & $1.71, \mathrm{~s}$ & & $1.71, \mathrm{~s}$ \\
\hline 21 & 18.3 & $1.67, \mathrm{~s}$ & 18.3 & $1.68, \mathrm{~s}$ & 18.3 & $1.68, \mathrm{~s}$ \\
\hline 22 & 27.4 & $1.01, \mathrm{~s}$ & 27.3 & $1.02, \mathrm{~s}$ & 27.3 & $1.01, \mathrm{~s}$ \\
\hline 23 & 23.3 & $1.24, \mathrm{~s}$ & 23.3 & $1.25, \mathrm{~s}$ & 23.3 & $1.24, \mathrm{~s}$ \\
\hline 24 & 30.1 & $2.15, \mathrm{~m}$ & 30.0 & $2.15, \mathrm{~m}$ & 30.0 & $2.15, \mathrm{~m}$ \\
\hline
\end{tabular}




\begin{tabular}{|c|c|c|c|c|c|c|}
\hline & & $2.06, \mathrm{~m}$ & & $2.06, \mathrm{~m}$ & & $2.06, \mathrm{~m}$ \\
\hline 25 & 125.6 & $4.90, \mathrm{~m}$ & 125.7 & $\mathrm{CD}_{3} \mathrm{OD}$ signal & 125.7 & $\mathrm{CD}_{3} \mathrm{OD}$ signal \\
\hline 26 & 137.3 & & 137.4 & & 137.3 & \\
\hline 27 & 40.8 & $1.96, \mathrm{~m}$ & 40.8 & $1.96, \mathrm{~m}$ & 40.7 & $1.96, \mathrm{~m}$ \\
\hline 28 & 16.4 & $1.47, \mathrm{~s}$ & 16.4 & $1.47, \mathrm{~s}$ & 16.4 & $1.47, \mathrm{~s}$ \\
\hline \multirow[t]{2}{*}{29} & 32.0 & $\begin{array}{l}2.52, \mathrm{dd} \quad(8.0 \\
14.0)\end{array}$ & 32.2 & $\begin{array}{l}2.53, \mathrm{dd} \quad(8.3, \\
14.1)\end{array}$ & 32.1 & $\begin{array}{l}2.52, \text { dd }(8.0 \\
14.1)\end{array}$ \\
\hline & & $2.47, \mathrm{~m}$ & & $2.47, \mathrm{~m}$ & & $2.48-2.43, \mathrm{~m}$ \\
\hline 30 & 120.7 & $5.16, \mathrm{~m}$ & 120.8 & $5.17, \mathrm{t}(7.3)$ & 120.8 & $5.16, \mathrm{~m}$ \\
\hline 31 & 135.7 & & 135.7 & & 135.6 & \\
\hline 32 & 26.3 & $1.69, \mathrm{~s}$ & 26.3 & $1.68, \mathrm{~s}$ & 26.3 & $1.68, \mathrm{~s}$ \\
\hline 33 & 18.3 & $1.67, \mathrm{~s}$ & 18.2 & $1.67, \mathrm{~s}$ & 18.3 & $1.66, \mathrm{~s}$ \\
\hline 34 & 27.5 & $2.06, \mathrm{~m}$ & 27.5 & $2.06, \mathrm{~m}$ & 27.5 & $2.06, \mathrm{~m}$ \\
\hline 35 & 125.1 & $5.06, \mathrm{~m}$ & 125.1 & $5.06, \mathrm{t}(7.0)$ & 125.4 & $5.06, \mathrm{~m}$ \\
\hline 36 & 132.1 & & 132.1 & & 132.1 & \\
\hline 37 & 26.0 & $1.65, \mathrm{~s}$ & 26.0 & $1.66, \mathrm{~s}$ & 26.0 & $1.66, \mathrm{~s}$ \\
\hline 38 & 17.8 & $1.56, \mathrm{~s}$ & 17.8 & $1.59, \mathrm{~s}$ & 17.8 & $1.58, \mathrm{~s}$ \\
\hline
\end{tabular}

Table S7. Comparison of ${ }^{1} \mathrm{H}$ NMR and ${ }^{13} \mathrm{C}$ NMR data for natural and synthetic oblongifolin $\mathrm{D}(7)^{7}$ in $\mathrm{CD}_{3} \mathrm{OD}+0.1 \%$ TFA- $d$

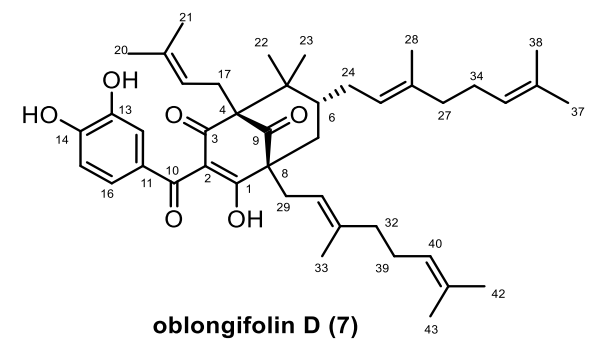

\begin{tabular}{lllll} 
& oblongifolin D isolated & \multicolumn{3}{c}{ oblongifolin D synthetic } \\
Position & $\delta \mathrm{C}(151 \mathrm{MHz})$ & $\delta \mathrm{H}\left(600 \mathrm{MH}_{\mathrm{z}}\right)$ & $\delta \mathrm{C}\left(101 \mathrm{MH}_{\mathrm{z}}\right)$ & $\delta \mathrm{H}\left(600 \mathrm{MH}_{\mathrm{z}}\right)$ \\
\hline 1 & 195.2 & & 195.3 & \\
2 & 118.0 & & 117.9 & \\
3 & 194.6 & & 194.8 & \\
4 & 68.2 & 68.1 & \\
5 & 49.2 & & 49.3 & $1.52, \mathrm{~m}$ \\
6 & 48.0 & $1.53, \mathrm{~m}$ & 47.8 & eq. $2.20, \mathrm{~m}$ \\
7 & 40.7 & eq. $2.20, \mathrm{~m}$ & 40.6 & ax. 2.11, m \\
& & ax. $2.11, \mathrm{~m}$ & & \\
8 & 66.5 & & 61.6 & \\
9 & 209.8 & & 209.8 & \\
10 & 195.9 & & 195.9 & $7.20, \mathrm{~d}(2.1)$ \\
11 & 129.7 & & 129.5 &
\end{tabular}




\begin{tabular}{|c|c|c|c|c|}
\hline 13 & 146.3 & & 146.2 & \\
\hline 14 & 152.7 & & 152.6 & \\
\hline 15 & 115.3 & $6.68, \mathrm{~d}(8.3)$ & 115.1 & $6.68, \mathrm{~d}(8.3)$ \\
\hline 16 & 125.6 & $6.98, \mathrm{dd}(8.3,2.1)$ & 125.4 & $6.98, \mathrm{dd}(8.3,2.2)$ \\
\hline \multirow[t]{2}{*}{17} & 27.3 & $2.73, \mathrm{dd}(9.0,13.0)$ & 27.1 & $2.73, \mathrm{dd}(9.1,13.6)$ \\
\hline & & $2.58, \mathrm{~m}$ & & $2.58, \mathrm{~m}$ \\
\hline 18 & 121.0 & 4.94, m & 120.9 & 4.97, m \\
\hline 19 & 135.9 & & 135.7 & \\
\hline 20 & 26.5 & $1.65, \mathrm{~s}$ & 26.4 & $1.66, \mathrm{~s}$ \\
\hline 21 & 18.4 & $1.68, \mathrm{~s}$ & 28.3 & $1.68, \mathrm{~s}$ \\
\hline 22 & 27.5 & $1.02, \mathrm{~s}$ & 27.4 & $1.02, \mathrm{~s}$ \\
\hline 23 & 23.4 & $1.23, \mathrm{~s}$ & 23.3 & $1.24, \mathrm{~s}$ \\
\hline \multirow[t]{2}{*}{24} & 30.2 & $2.15, \mathrm{~m}$ & 30.0 & $2.15, \mathrm{~m}$ \\
\hline & & $2.07, \mathrm{~m}$ & & $2.08, \mathrm{~m}$ \\
\hline 25 & 125.8 & $4.87, \mathrm{~m}$ & 125.7 & $4.88, \mathrm{~m}$ \\
\hline 26 & 137.5 & & 137.4 & \\
\hline 27 & 41.0 & $1.98, \mathrm{~m}$ & 40.9 & $1.98, \mathrm{~m}$ \\
\hline 28 & 16.6 & $1.48, \mathrm{~s}$ & 16.4 & $1.48, \mathrm{~s}$ \\
\hline 29 & 32.0 & $2.52, \mathrm{~m}$ & 31.9 & $2.52, \mathrm{~m}$ \\
\hline 30 & 120.7 & $5.16, \mathrm{~m}$ & 120.5 & $5.17, \mathrm{t}(7.2)$ \\
\hline 31 & 139.3 & & 139.1 & \\
\hline 32 & 41.1 & $1.98, \mathrm{~m}$ & 41.0 & $1.98, \mathrm{~m}$ \\
\hline 33 & 17.0 & $1.69, \mathrm{~s}$ & 16.9 & $1.69, \mathrm{~s}$ \\
\hline 34 & 27.8 & $2.05, \mathrm{~m}$ & 27.7 & $2.05, \mathrm{~m}$ \\
\hline 35 & 125.4 & $5.06, \mathrm{~m}$ & 125.3 & $5.06, \mathrm{~m}$ \\
\hline 36 & 132.2 & & 132.1 & \\
\hline 37 & 26.0 & $1.58, \mathrm{~s}$ & 26.0 & $1.58, \mathrm{~s}$ \\
\hline 38 & 17.9 & $1.53, \mathrm{~s}$ & 17.8 & $1.53, \mathrm{~s}$ \\
\hline 39 & 27.7 & $2.05, \mathrm{~m}$ & 27.5 & $2.05, \mathrm{~m}$ \\
\hline 40 & 125.4 & $5.06, \mathrm{~m}$ & 125.3 & $5.06, \mathrm{~m}$ \\
\hline 41 & 132.3 & & 132.2 & \\
\hline 42 & 26.1 & $1.65, \mathrm{~s}$ & 26.0 & $1.67, \mathrm{~s}$ \\
\hline 43 & 17.9 & $1.59, \mathrm{~s}$ & 17.8 & $1.58, \mathrm{~s}$ \\
\hline
\end{tabular}




\section{MTT assay of cell viability and Cytotoxicity data}

The 3-(4,5-dimethylthiazol-2-yl)-2,5-diphenyltetrazolium bromide (MTT) powder and dimethyl sulfoxide (DMSO) purchased from Sigma-Aldrich (St. Louis, USA). HeLa cells, HL-7702 cells, HCT-116 cells were obtained from ATCC (Manassas, USA) and cultured in DMEM (Invitrogen, Carlsbad, USA) containing 10\% FBS (Invitrogen, Carlsbad, USA). SGC-7901 cells were kindly provided by Department of Traditional Chinese Medicine of Changzheng Hospital (Second Military Medical University, Shanghai, China) and incubated in RPMI 1640 medium supplied with 10\% fetal bovine serum. All cell lines were maintained at $37^{\circ} \mathrm{C}$ in a humidified atmosphere with $5 \% \mathrm{CO}_{2}$.

Cells were seeded in 96-well plates and treated with different compounds at various concentrations $(1.25 \mu \mathrm{M}, 2.5$ $\mu \mathrm{M}, 5 \mu \mathrm{M}, 10 \mu \mathrm{M}$ and $20 \mu \mathrm{M})$ for $72 \mathrm{~h}$. Cells were incubated with $100 \mu \mathrm{L}$ of fresh medium containing $10 \mu \mathrm{L}$ MTT (5 $\mathrm{mg} / \mathrm{mL}$ ) at $37^{\circ} \mathrm{C}$ for $4 \mathrm{~h}$ and subsequent dissolving of formazan crystals in DMSO. Absorbance was measured at $570 \mathrm{~nm}$ using a microplate reader. The inhibition rates were calculated using the following formula: $\left[1-\left(\mathrm{T}_{72}-\mathrm{C}_{0}\right) /\left(\mathrm{C}_{72}-\mathrm{C}_{0}\right)\right] \times$ $100 \% . \mathrm{IC}_{50}$ is defined as the concentration of a compound inhibiting $50 \%$ of cell growth. Each $\mathrm{IC}_{50}$ test was performed in triplicate and repeated at least three times. All statistical analyses were performed by using SPSS 15.0 statistical software (SPSS). Quantified results were given as the means \pm SD from three independent experiments unless otherwise indicated. Comparisons were performed with probit regression analysis and differences were considered significant at $p<0.05$.

Table S8. Cytotoxicity data against the tested cell lines and SARs study ${ }^{\text {a }}$

$\begin{array}{llll}\text { Compound } & \mathrm{IC}_{50}(\mathrm{HeLa}) & \mathrm{IC}_{50}(\mathrm{SGC7901}) & \mathrm{IC}_{50}(\mathrm{HCT} 116) \\ \mathbf{1} & 11.97 \pm 3.39 & 5.42 \pm 0.30 & 6.87 \pm 1.82 \\ \mathbf{2} & 13.71 \pm 1.94 & 6.93 \pm 1.39 & 9.22 \pm 2.06 \\ \mathbf{3} & 7.65 \pm 0.18 & 4.20 \pm 0.33 & 5.96 \pm 0.13 \\ \mathbf{4} & 11.69 \pm 5.69 & 5.48 \pm 0.78 & 8.60 \pm 1.63 \\ \mathbf{7} & 8.09 \pm 1.81 & 4.05 \pm 0.39 & 4.92 \pm 1.17 \\ \mathbf{2 1} & 4.69 \pm 1.69 & 3.01 \pm 0.20 & 2.44 \pm 1.22 \\ \mathbf{1 5 b} & 6.64 \pm 1.89 & 5.60 \pm 0.68 & 6.46 \pm 1.36 \\ \mathbf{1 5 e} & 9.69 \pm 2.57 & 6.89 \pm 0.14 & 9.14 \pm 2.03 \\ \mathbf{1 5 f} & 11.65 \pm 4.61 & 8.5 \pm 2.20 & 10.91 \pm 2.50 \\ \text { Taxol } & 0.047 \pm 0.02 & 0.051 \pm 0.01 & 0.038 \pm 0.03\end{array}$

${ }^{\text {a }} C_{50}$ (mean values $\pm \mathrm{SD}, \mathrm{n}=3$ ) is the $50 \%$ inhibitory concentration of the compound against cell growth, reported in $\mu \mathrm{M}$.

For compounds not listed in the table: $\mathrm{IC}_{50}>20 \mu \mathrm{M}$.

\section{References}

1. Perry, M. A.; Hill, R. R.; Leong, J. J.; Rychnovsky, S. D. Org. Lett. 2015, 17, 3268-3271.

2. Tang, Q.; Sen, S. E. Tetrahedron Lett. 1998, 39, 2249-2252.

3. Sowell, C. G.; Wolin, R. L.; Little, R. D. Tetrahedron Lett. 1990, 31, 485-488.

4. Piccinelli, A. L.; Cuesta-Rubio, O.; Chica, M. B.; Mahmood, N.; Pagano, B.; Pavone, M.; Barone, V.; Rastrelli, L. Tetrahedron 2005, 61, 8206-8211.

5. Fu, W. W.; Wu, M; Zhu, L. L.; Lao, Y. Z.; Wang, L. P.; Tan, H. S.; Yuan, Q. H.; Xu, H. X. Rsc Adv. 2015, 5, $78259-78267$.

6. Nilar; Nguyen, L.-H. D.; Venkatraman, G.; Sim, K.-Y.; Harrison, L. J. Phytochem. 2005, 66, 1718-1723.

7. Hamed, W.; Brajeul, S.; Mahuteau-Betzer, F.; Thoison, O.; Mons, S.; Delpech, B.; Hung, N. V.; Sévenet, T.; Marazano, C. J. Nat. Prod. 2006, 69, 774-777.

8. Biber, N., Mows, K. \& Plietker, B. Nat. Chem. 2011, 3, 938. 


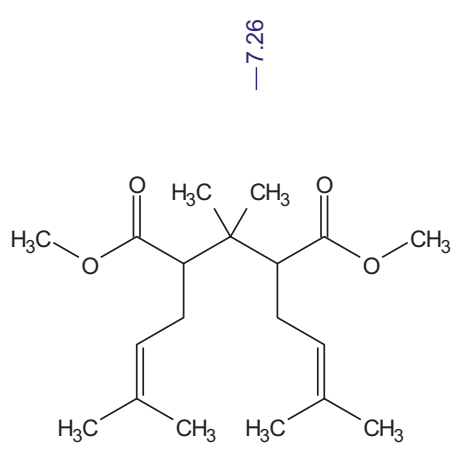

$10 a$

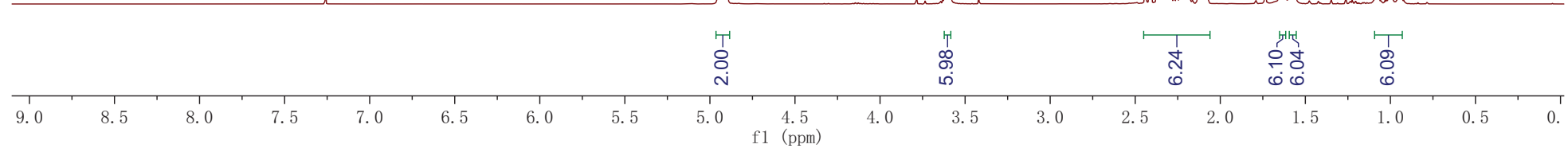

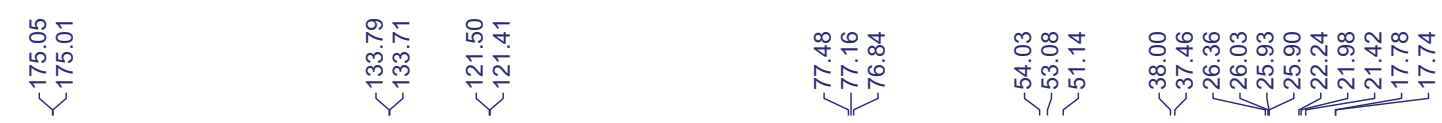<smiles>COC(=O)C(CC=C(C)C)C(C)(C)C(CC=C(C)C)C(=O)OC</smiles>

$10 a$ 
<smiles>CC(C)=CCC(CO)C(C)(C)C(CO)CC=C(C)C</smiles>

S1

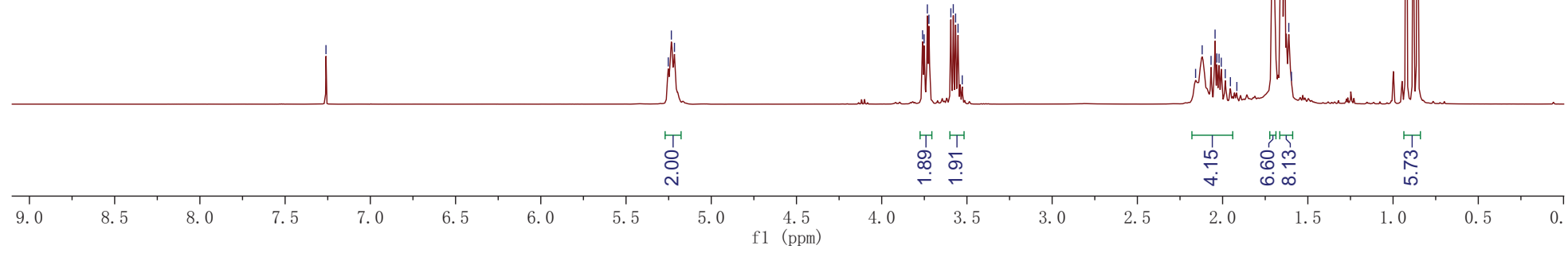

मूळ छा

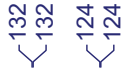

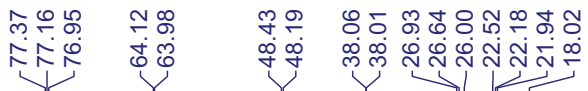<smiles>CC(C)=CCC(CO)C(C)(C)C(CO)CC=C(C)C</smiles>

S1 
<smiles>COC(=O)CC(C)(C)C(CC=C(C)C)C(=O)OC</smiles>

s3

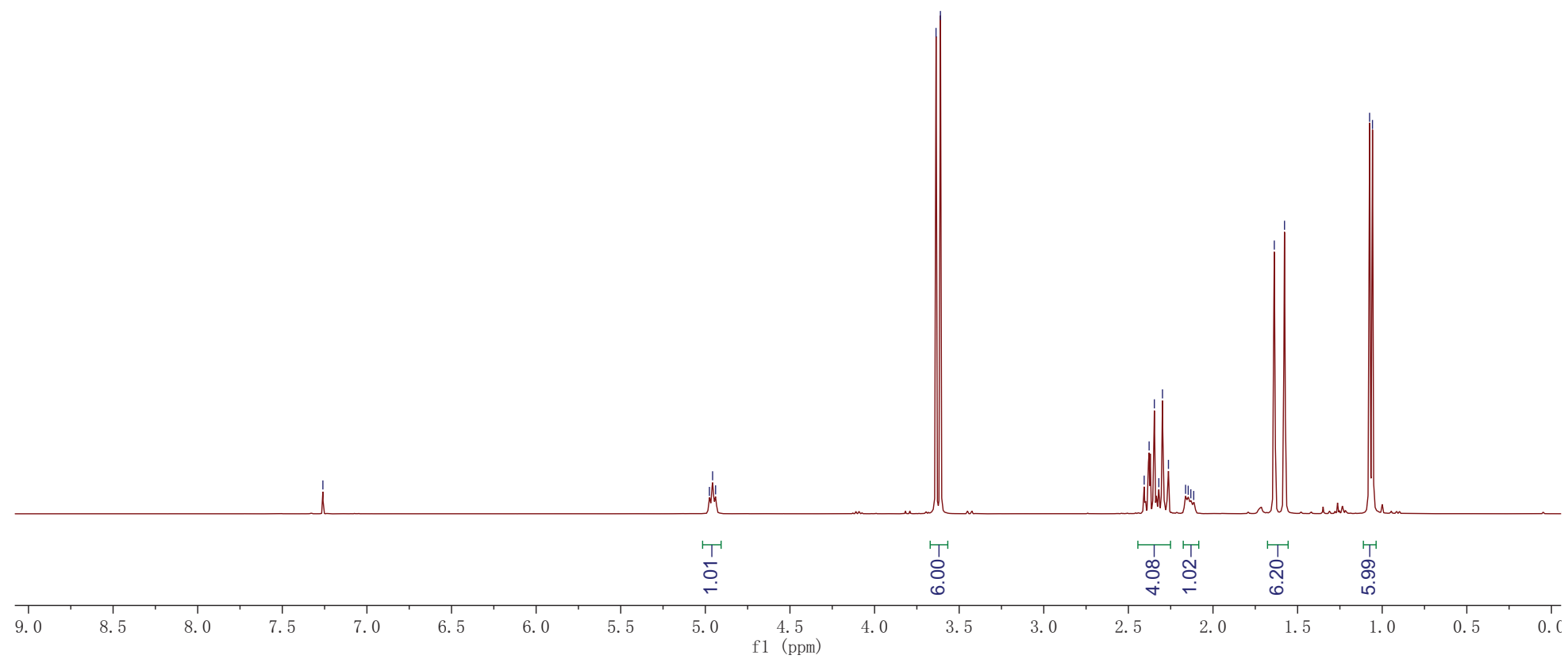

管<smiles>COC(=O)CC(C)(C)C(CC=C(C)C)C(=O)OC</smiles>

S3 
<smiles>CC(=O)C(CC=C(C)C)C(C)(C)C(CCl)CC=C(C)C</smiles>

$12 \mathrm{a}$
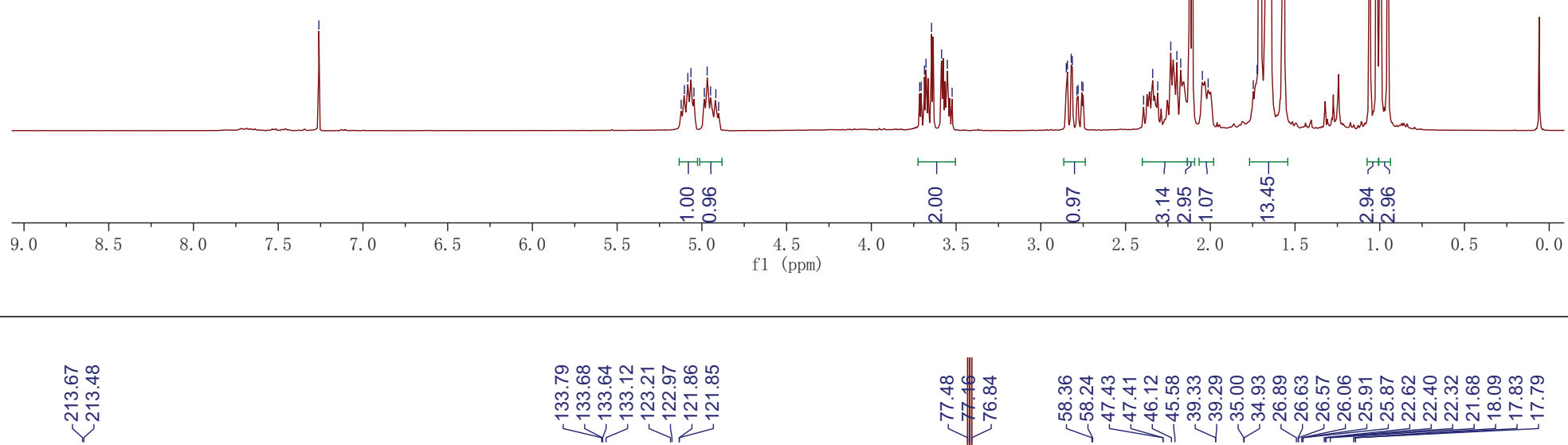

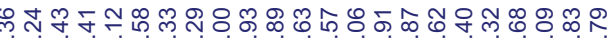

色

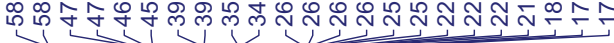<smiles>CC(=O)C(CC=C(C)C)C(C)(C)C(CCl)CC=C(C)C</smiles>

$12 \mathrm{a}$

$\begin{array}{rrrrrrrrrrrrr}220 & 210 & 200 & 190 & 180 & 170 & 160 & 150 & 140 & 130 & 120 & 110 & 100 \\ \mathrm{f} 1(\mathrm{ppm})\end{array}$




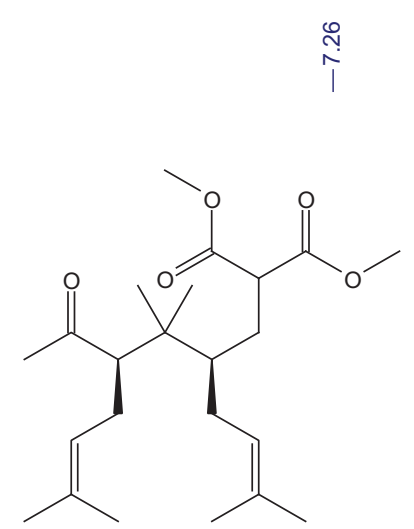

13a (cis)
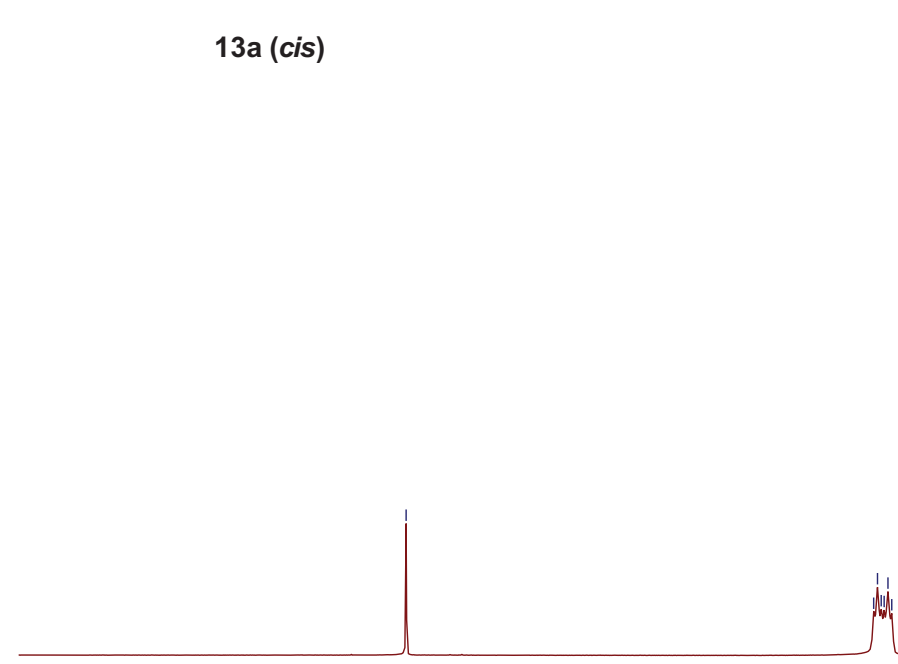

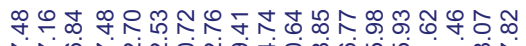

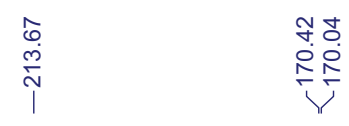

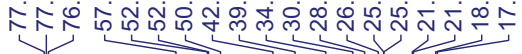

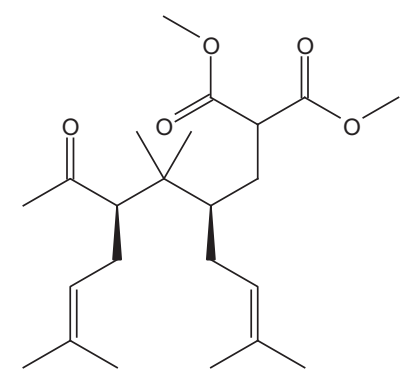

13a (cis)
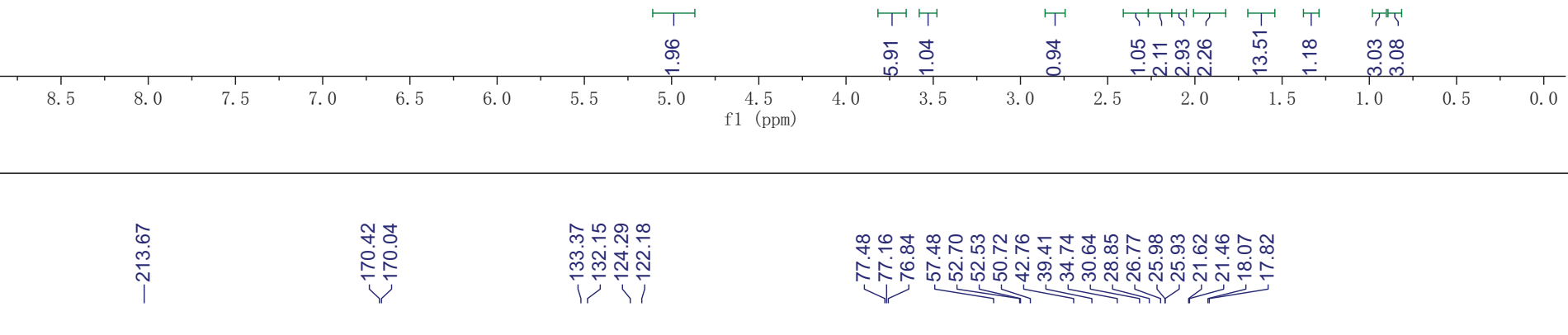

I 
<smiles>COC(=O)C(C[C@@H](CC=C(C)C)C(C)(C)[C@H](CC=C(C)C)C(C)=O)C(=O)OC</smiles>

13a (trans)
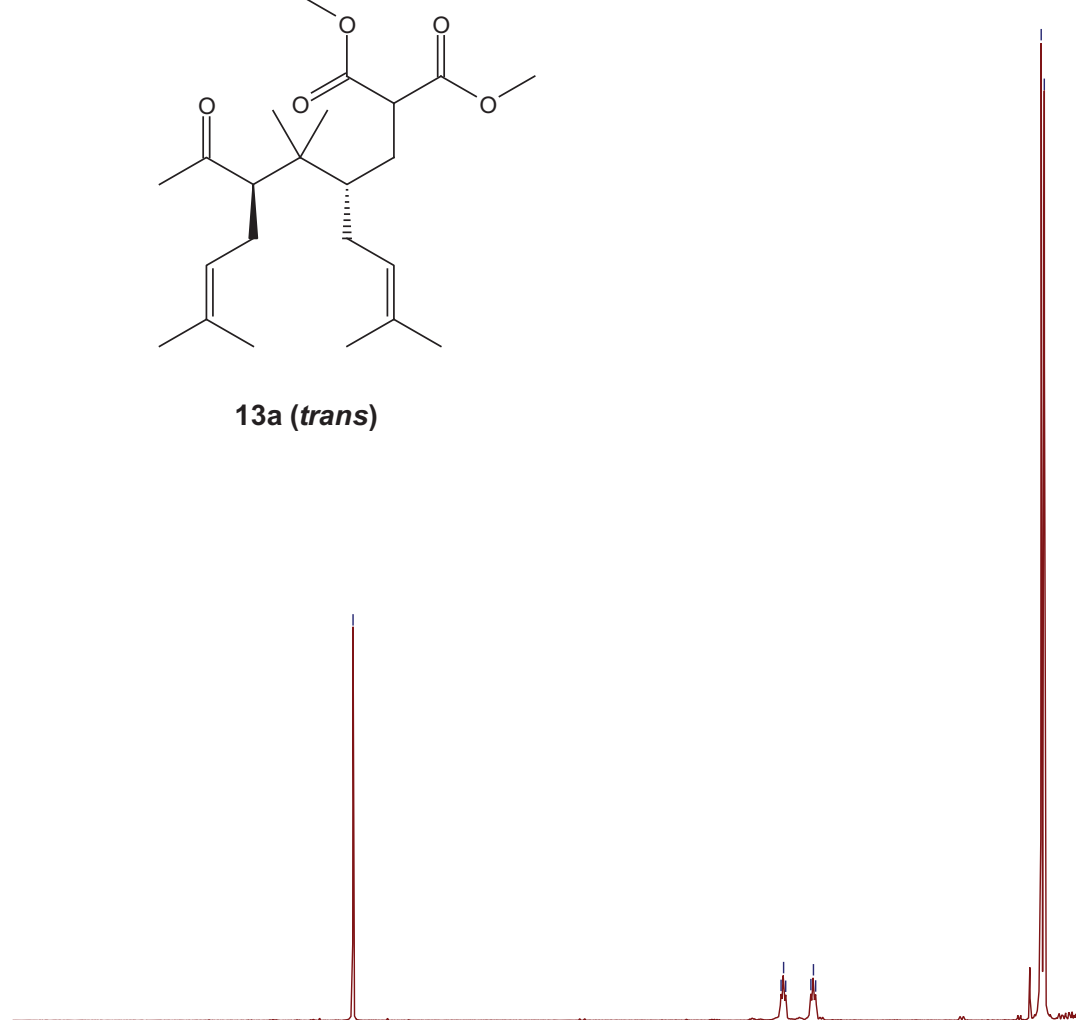

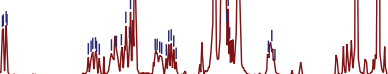

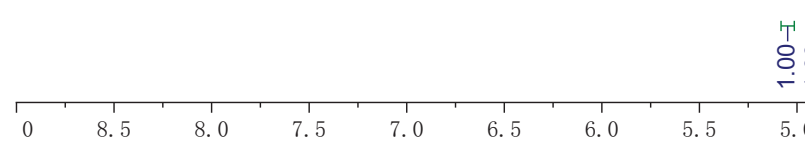

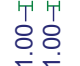

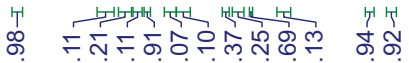

管<smiles>COC(=O)C(C[C@@H](CC=C(C)C)C(C)(C)[C@H](CC=C(C)C)C(C)=O)C(=O)OC</smiles>

13a (trans)

유융ำ

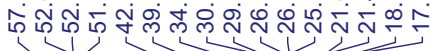

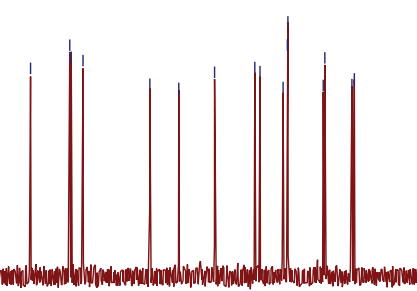


<smiles>COC(=O)C(CC=C(C)C)(CC(CC=C(C)C)C(C)(C)C(CC=C(C)C)C(C)=O)C(=O)OC</smiles>

$14 a$

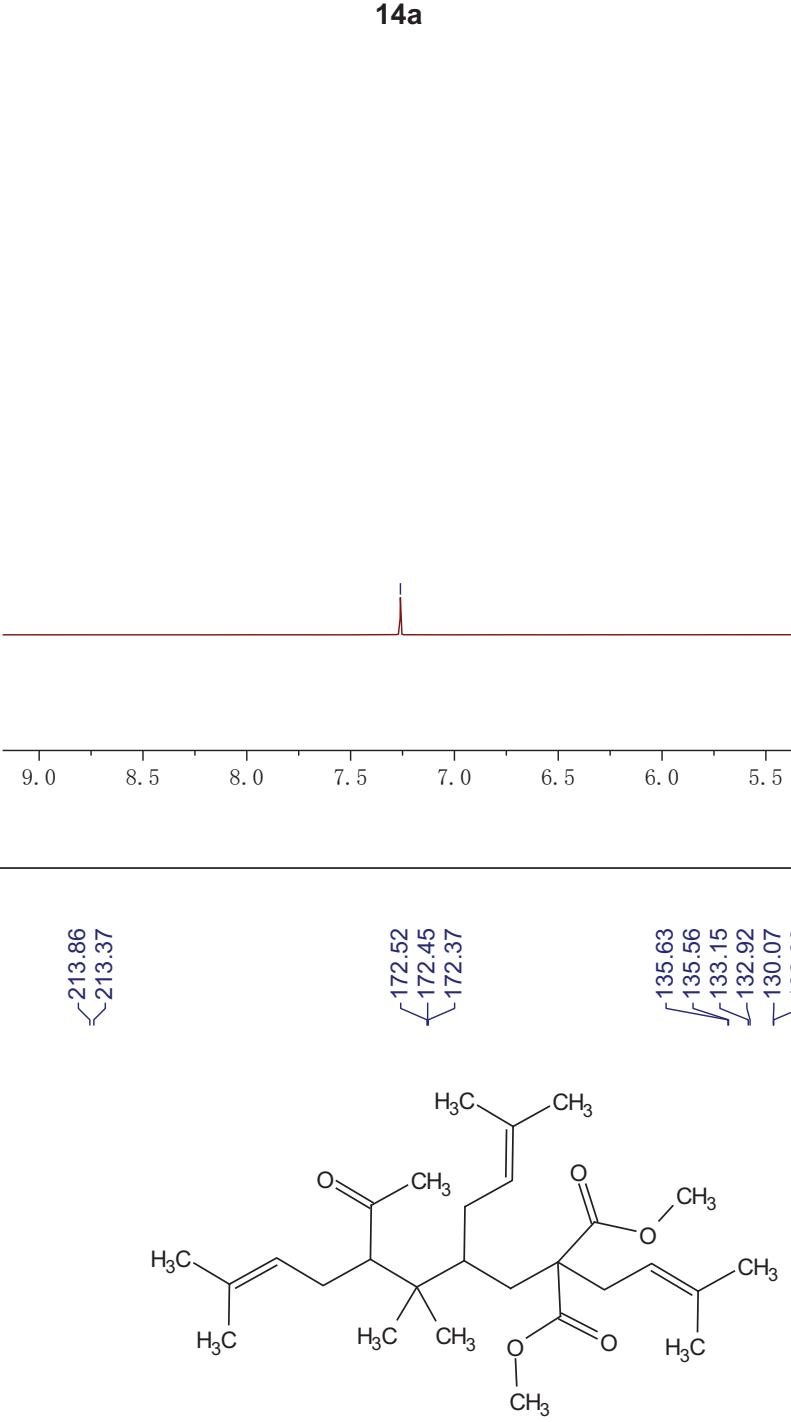

$14 a$

临

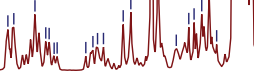
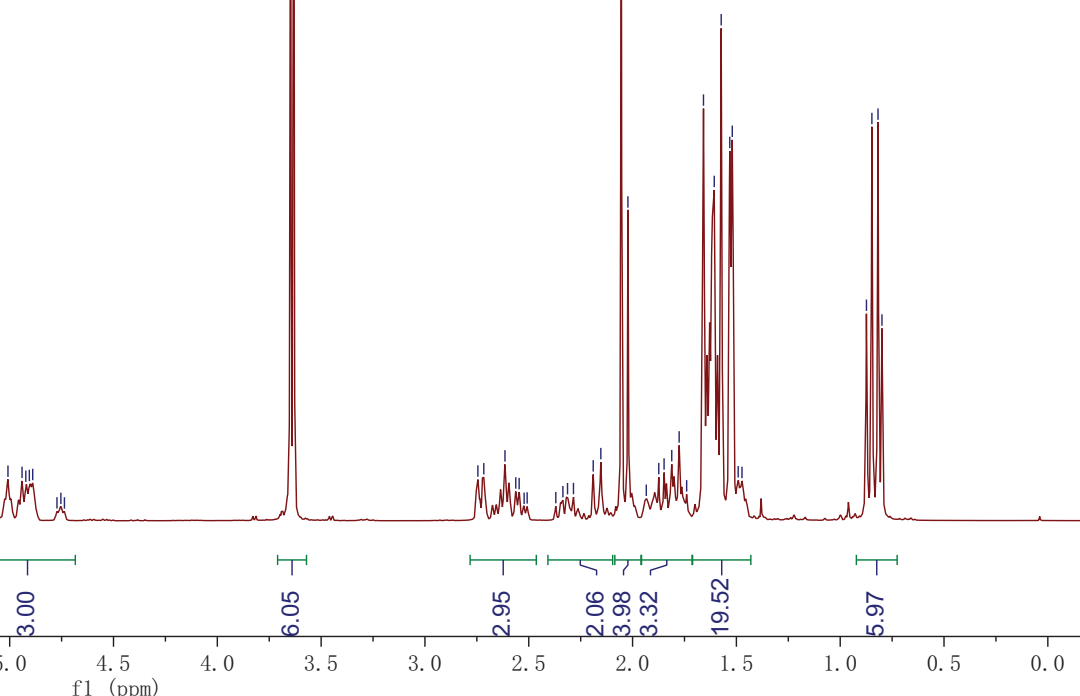
<smiles>COC(=O)C(C/C=C(\C)CCC=C(C)C)(CC(CC=C(C)C)C(C)(C)C(CC=C(C)C)C(C)=O)C(=O)OC</smiles>

$14 b$

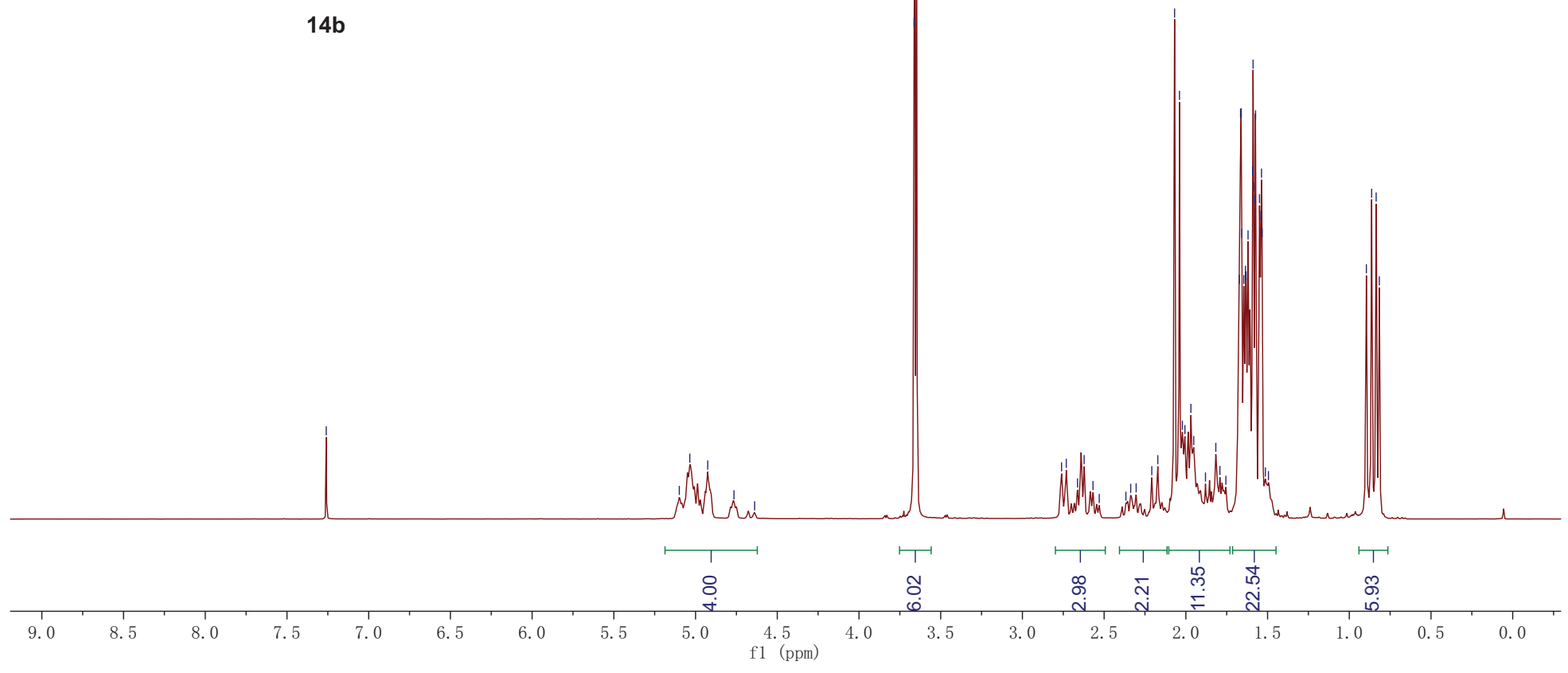

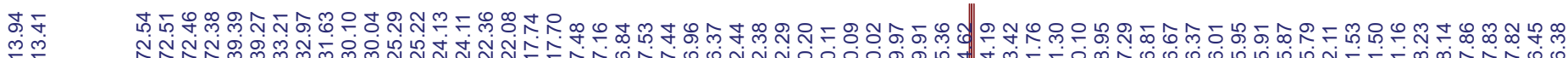

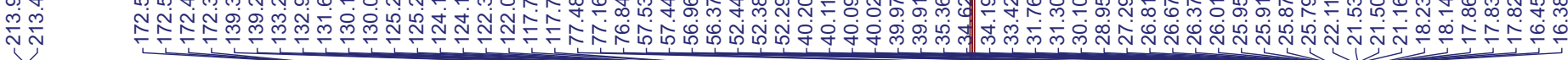<smiles>COC(=O)C(C/C=C(\C)CCC=C(C)C)(CC(CC=C(C)C)C(C)(C)C(CC=C(C)C)C(C)=O)C(=O)OC</smiles>

$14 b$ 


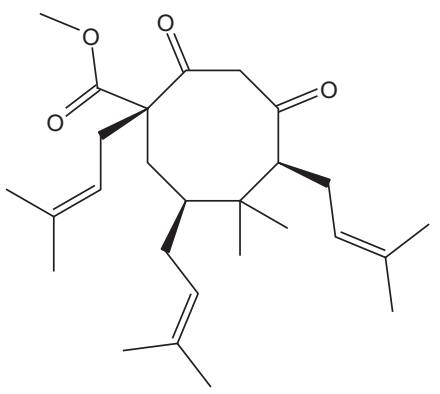

16

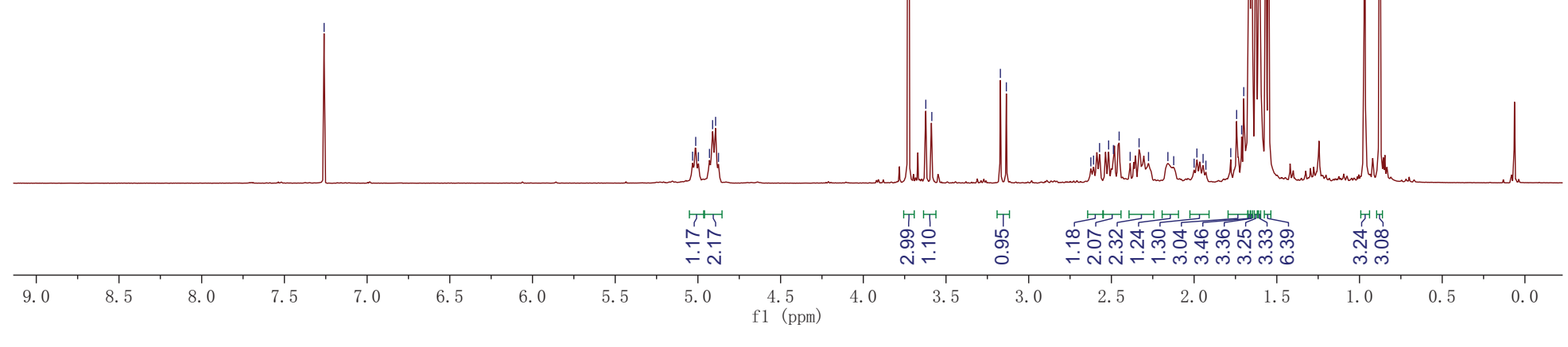

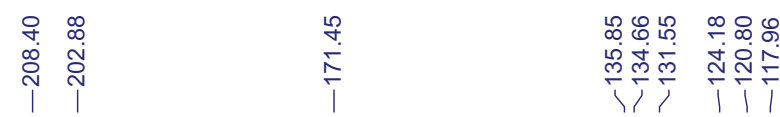

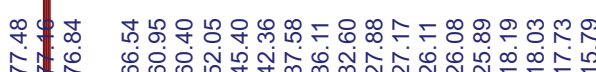

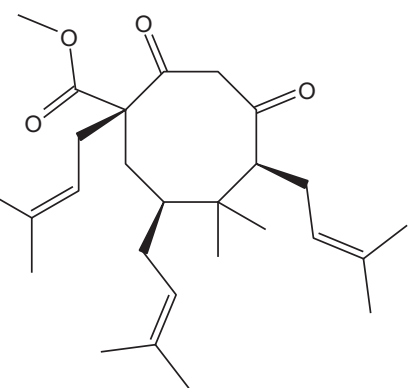

16

许

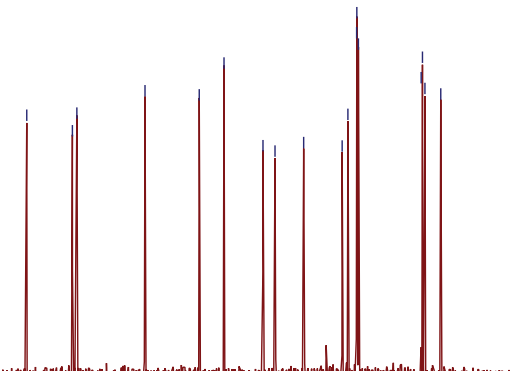



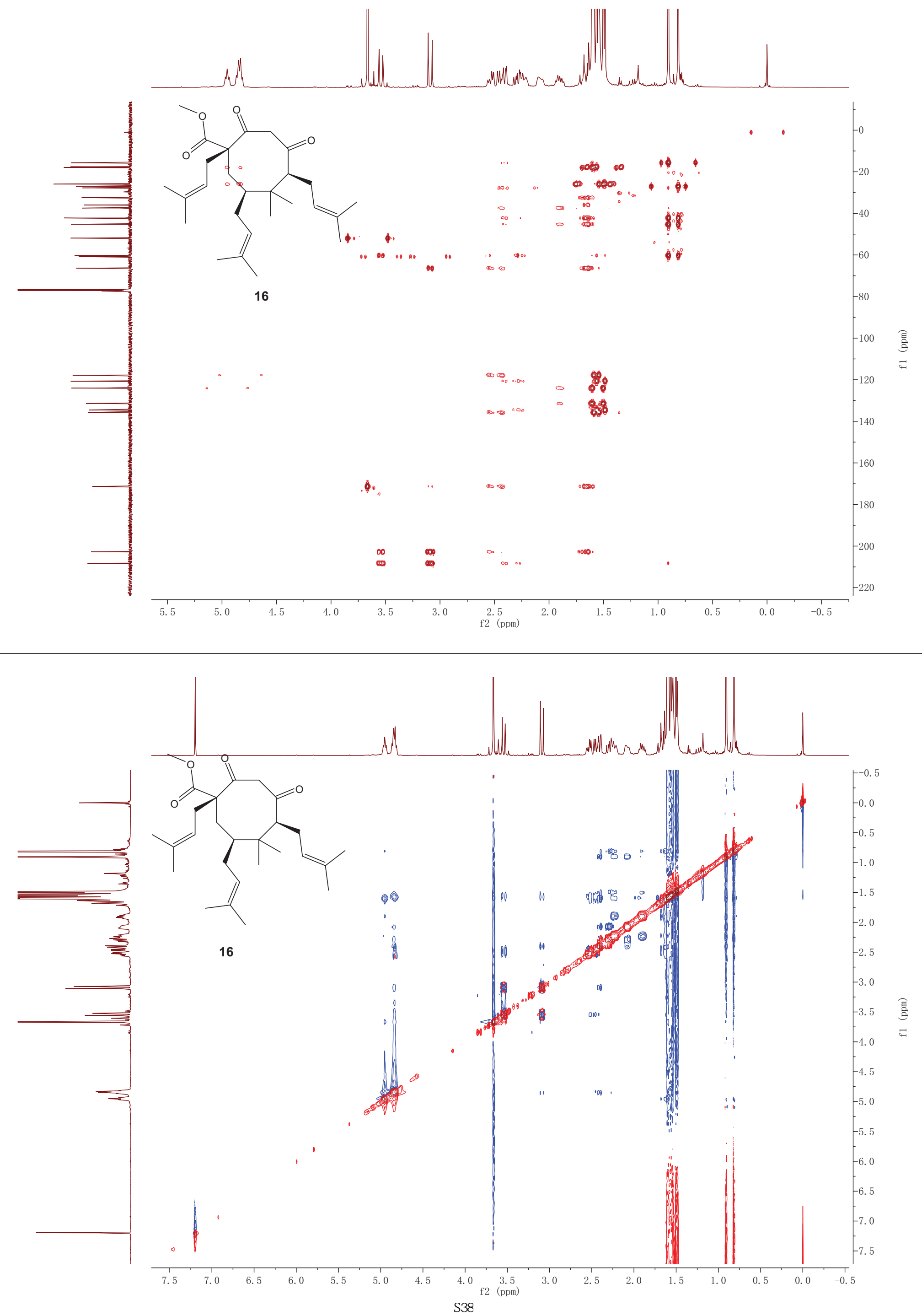

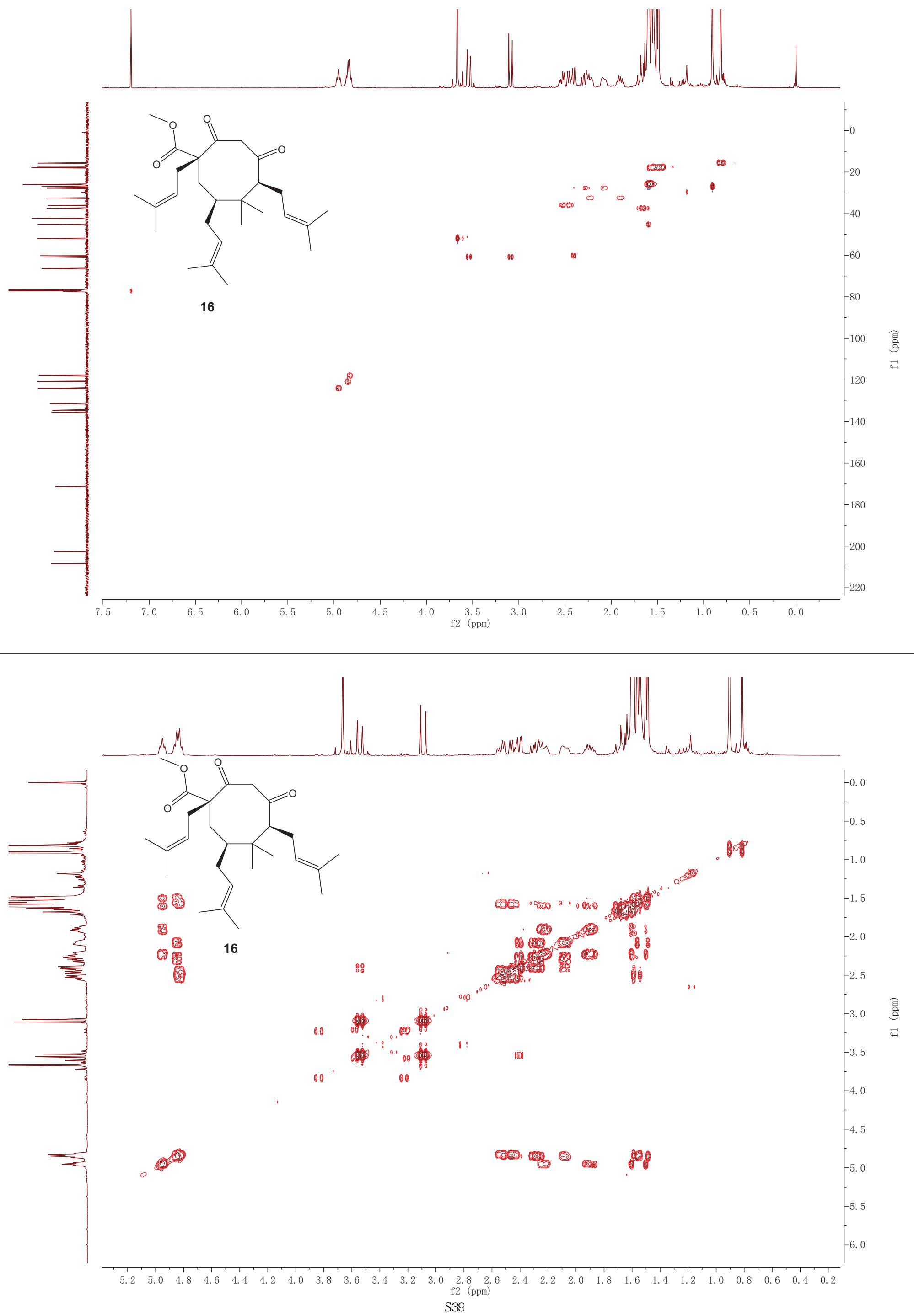

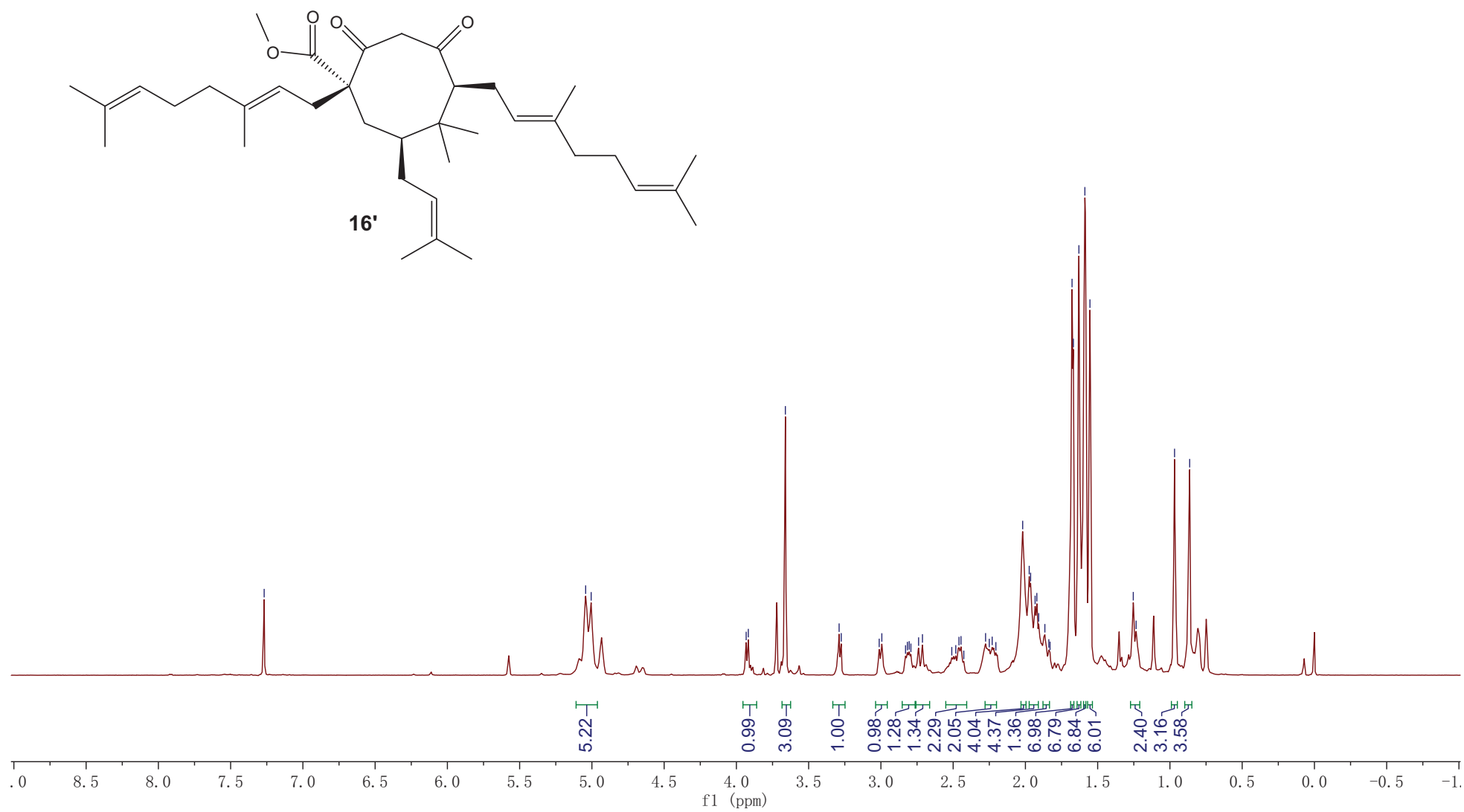

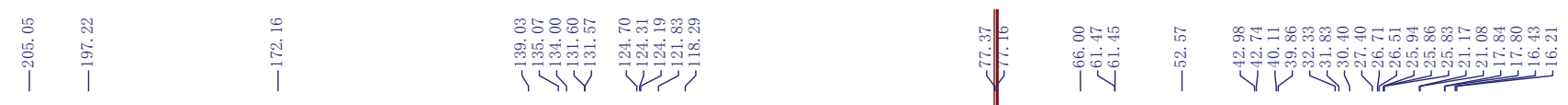

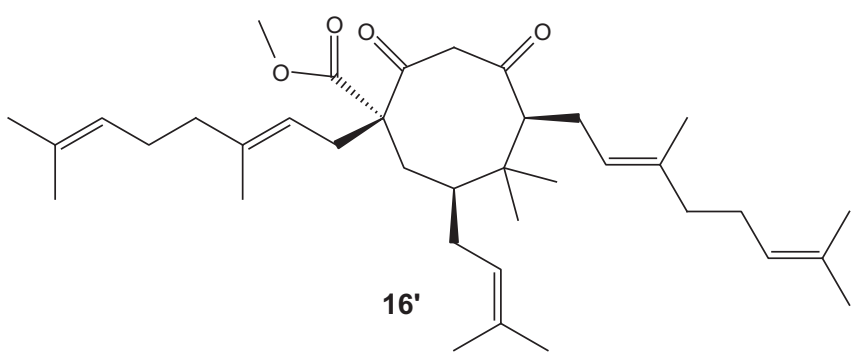

\begin{tabular}{|c|c|c|c|c|c|c|c|c|c|c|c|c|c|c|c|c|c|c|c|c|c|c|}
\hline 210 & 200 & 190 & 180 & 170 & 160 & 150 & 140 & 130 & 120 & 110 & $\mathrm{f} 1 \begin{array}{c}100 \\
(\mathrm{ppm})\end{array}$ & 90 & 80 & 70 & 60 & 50 & 40 & 30 & 20 & 10 & 0 & -10 \\
\hline
\end{tabular}




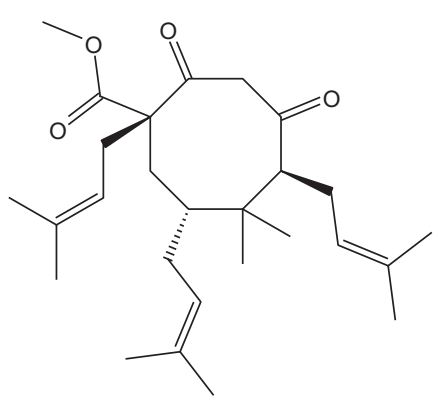

17
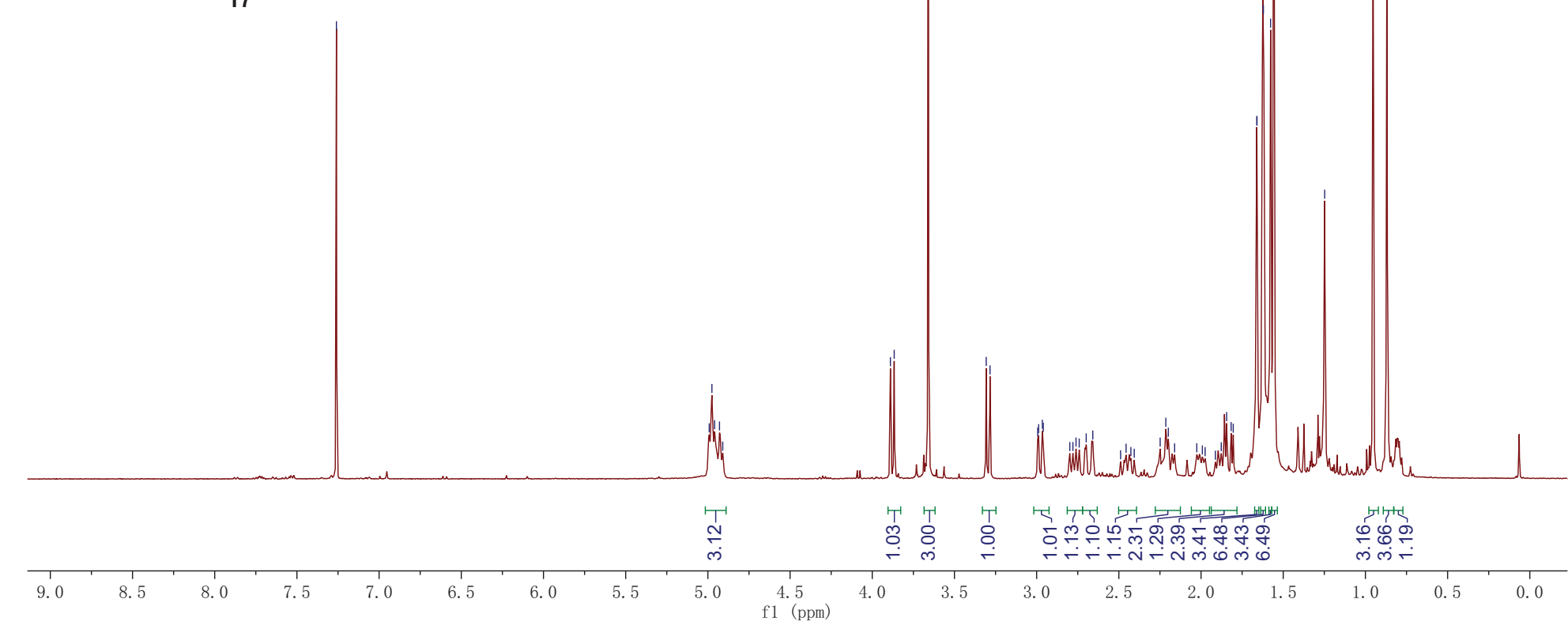

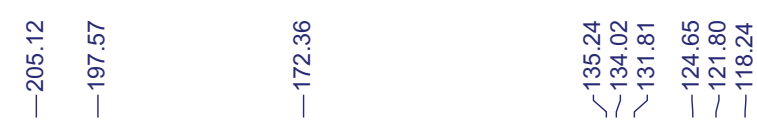

$\underbrace{\infty}$

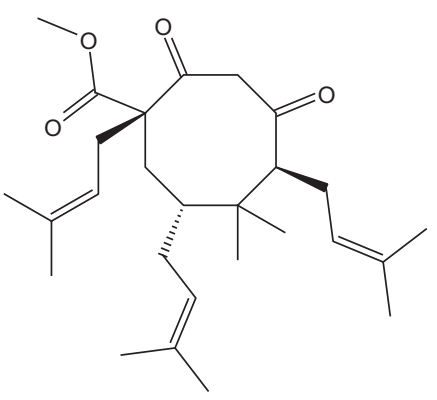

17

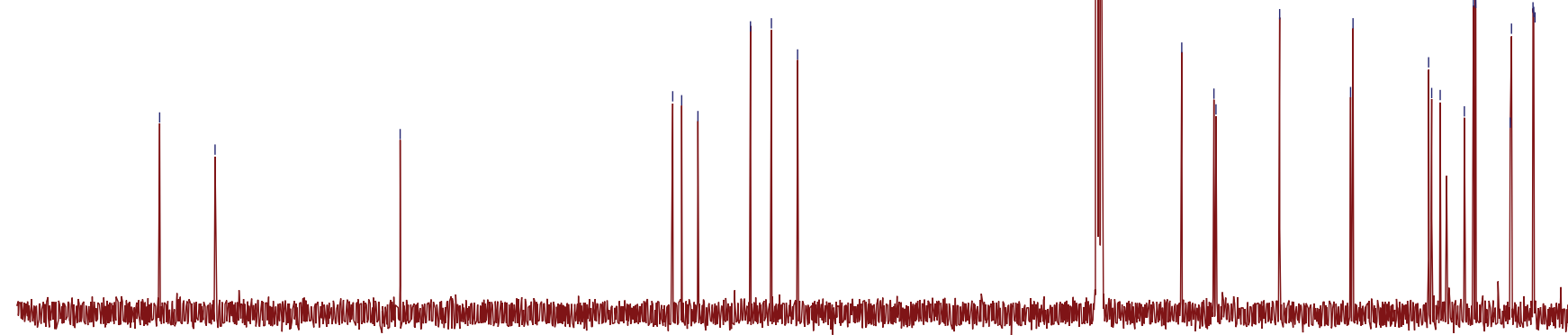



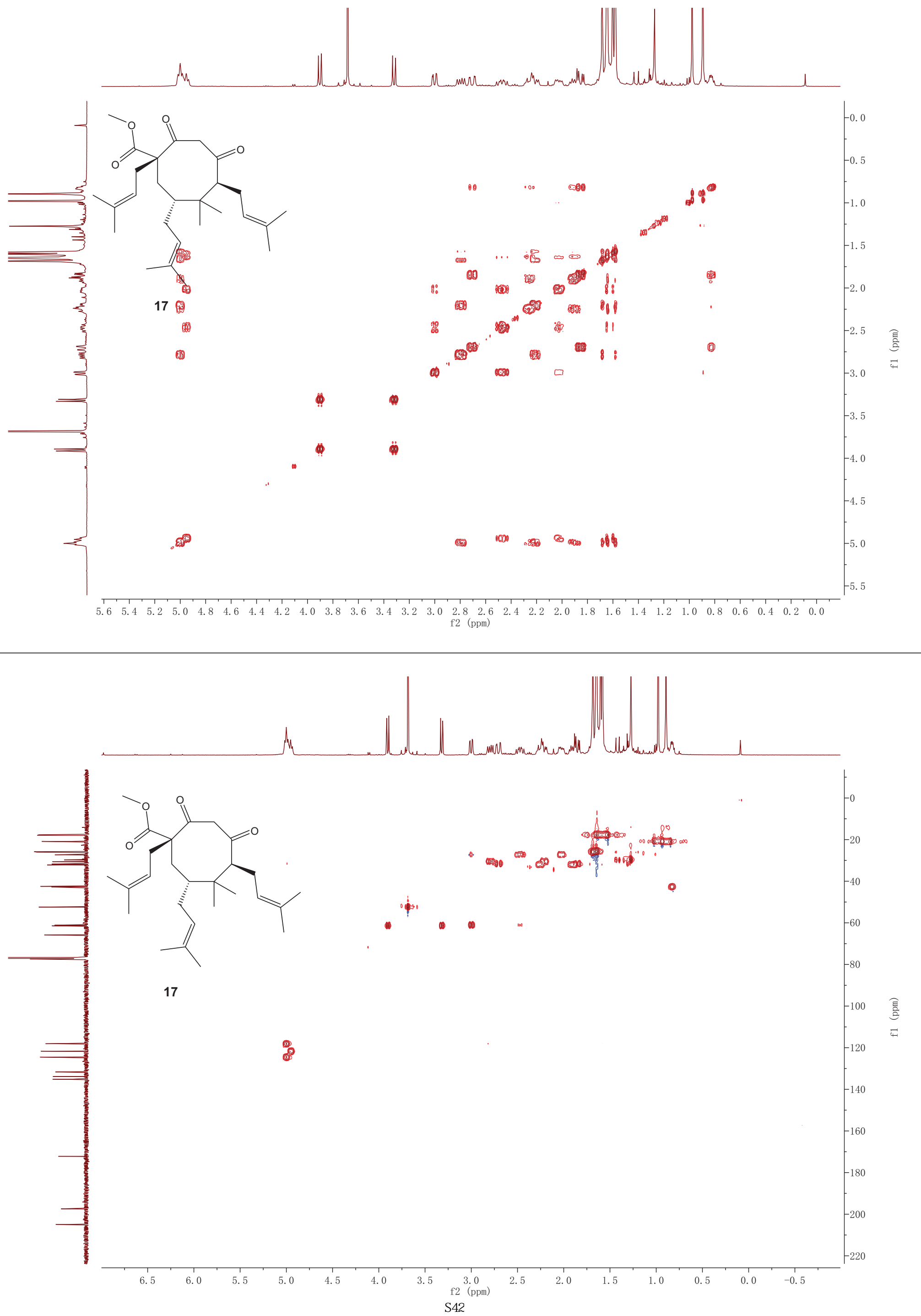

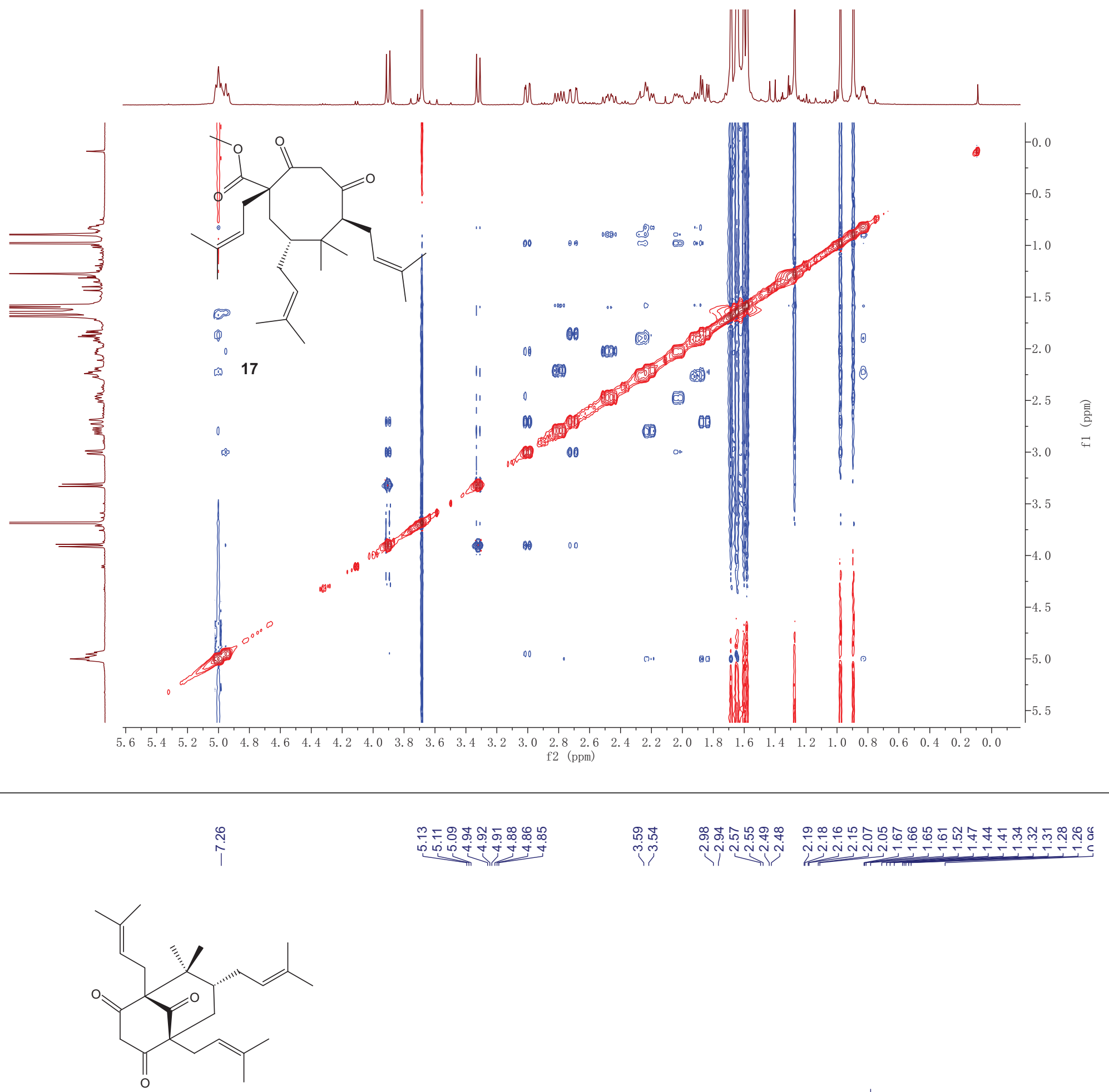

$15 a$

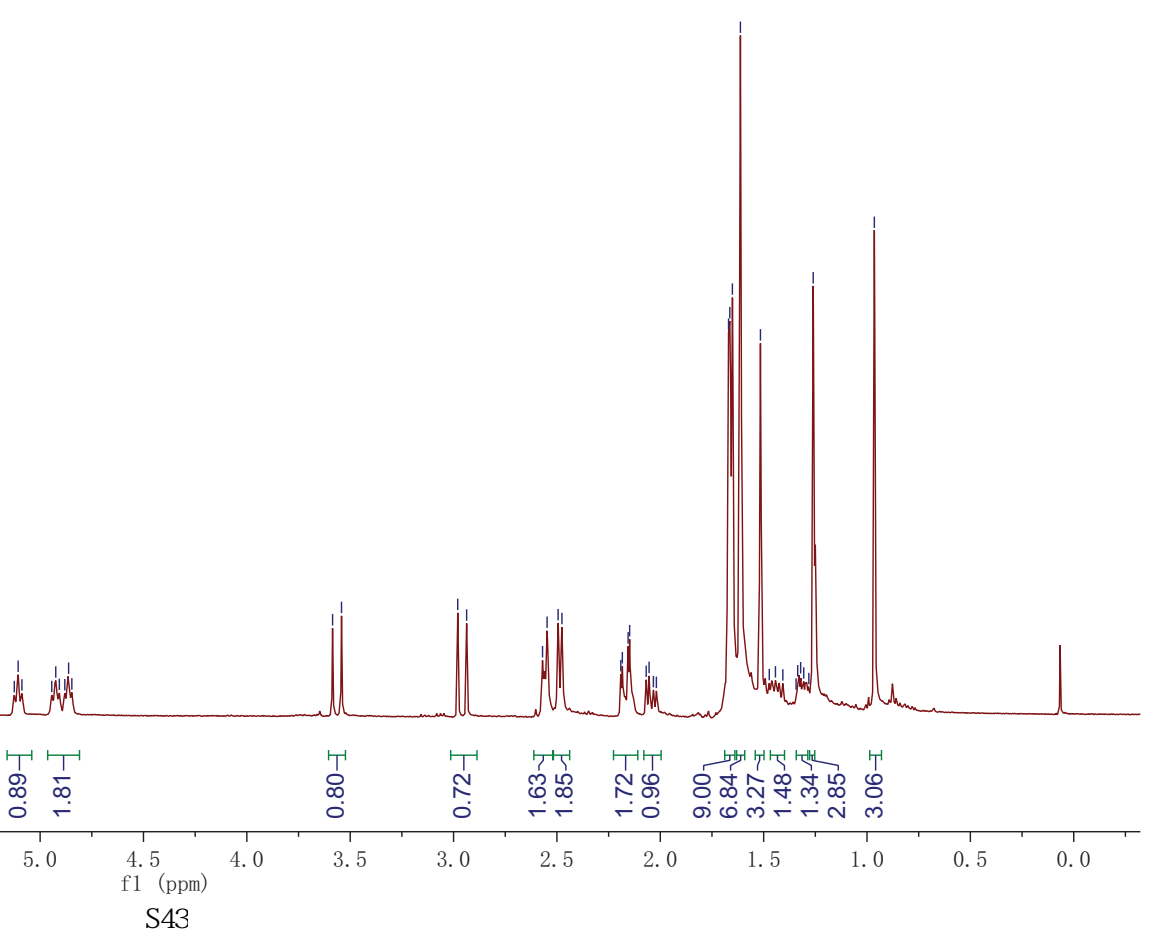




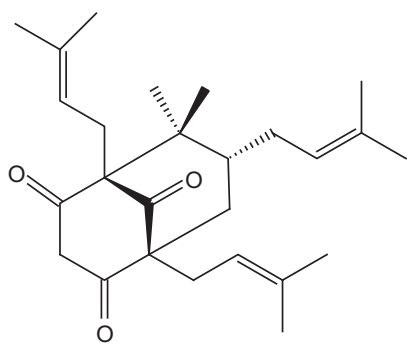

$15 a$

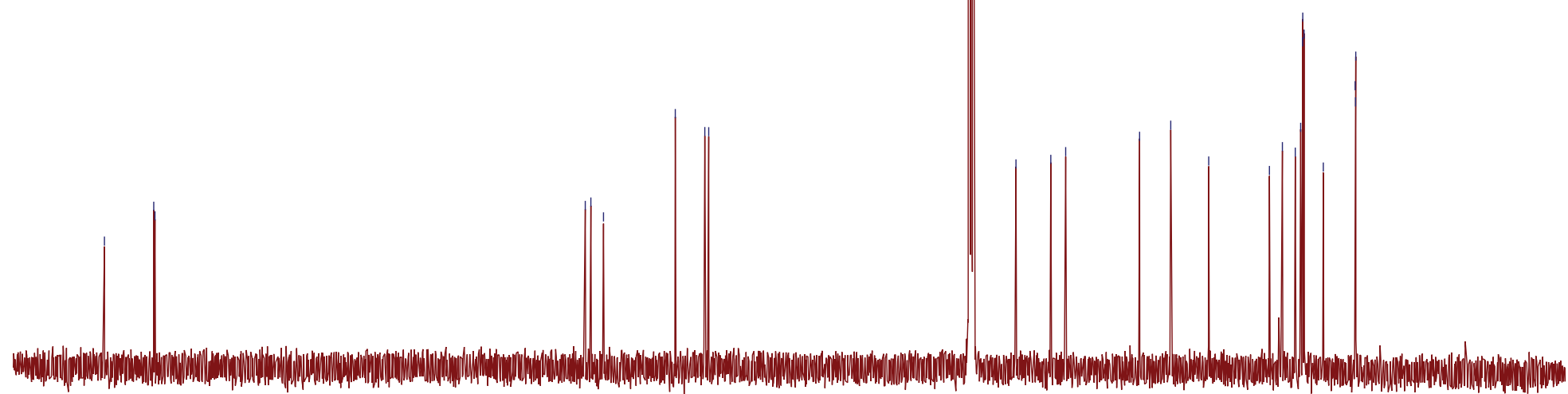

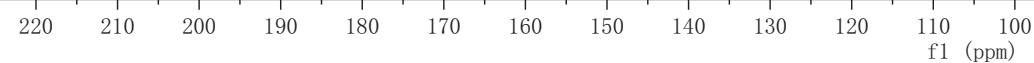

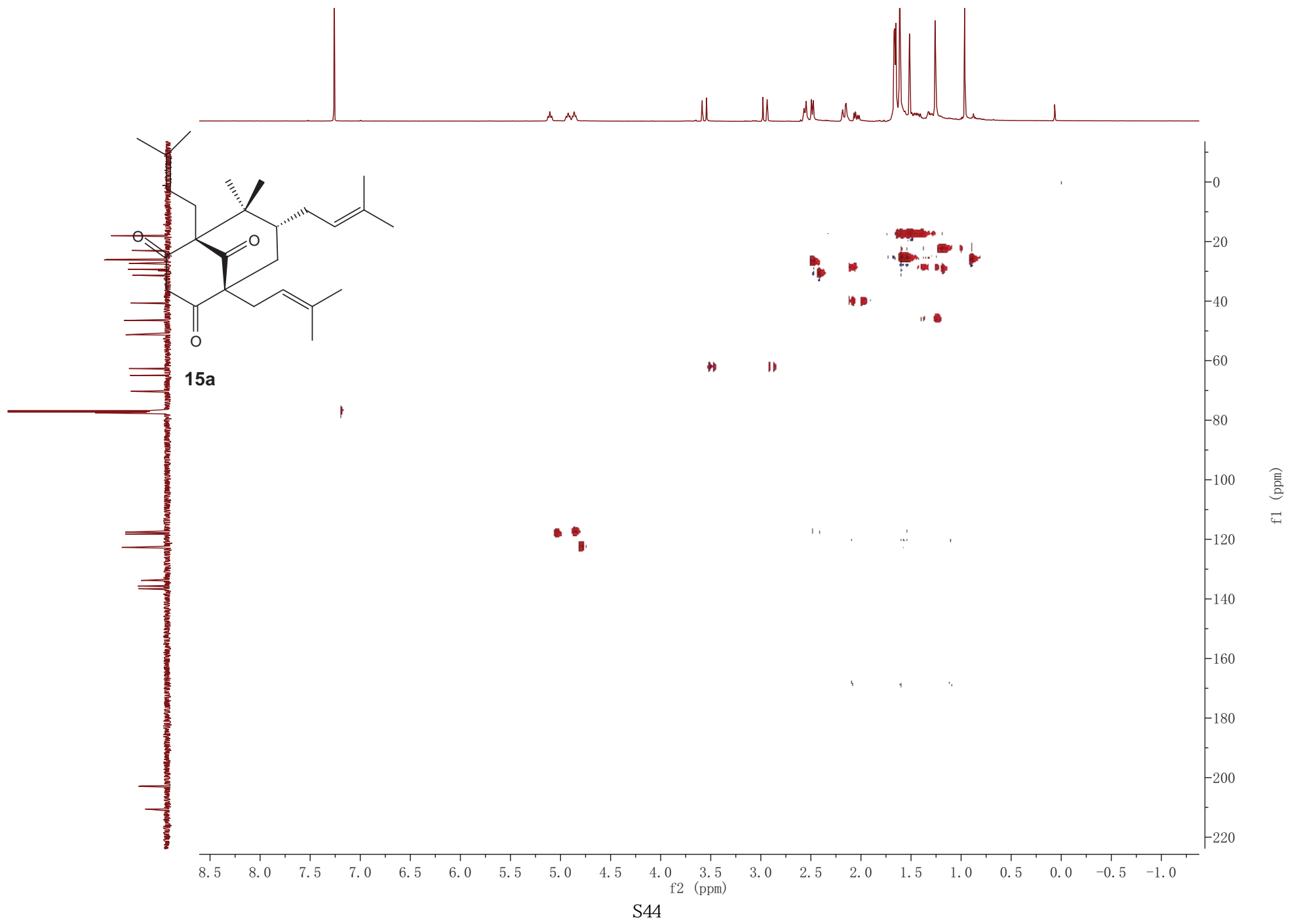



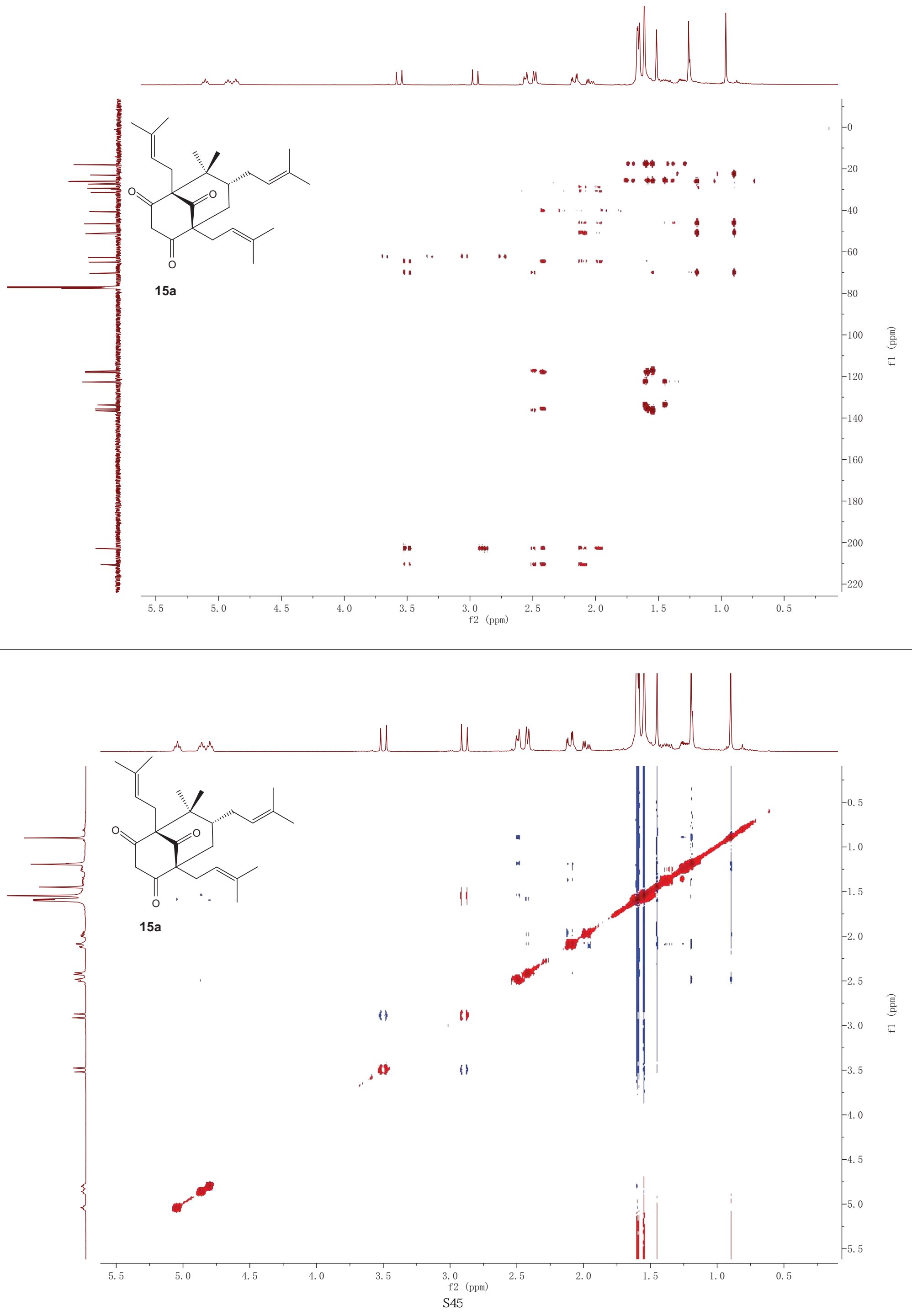

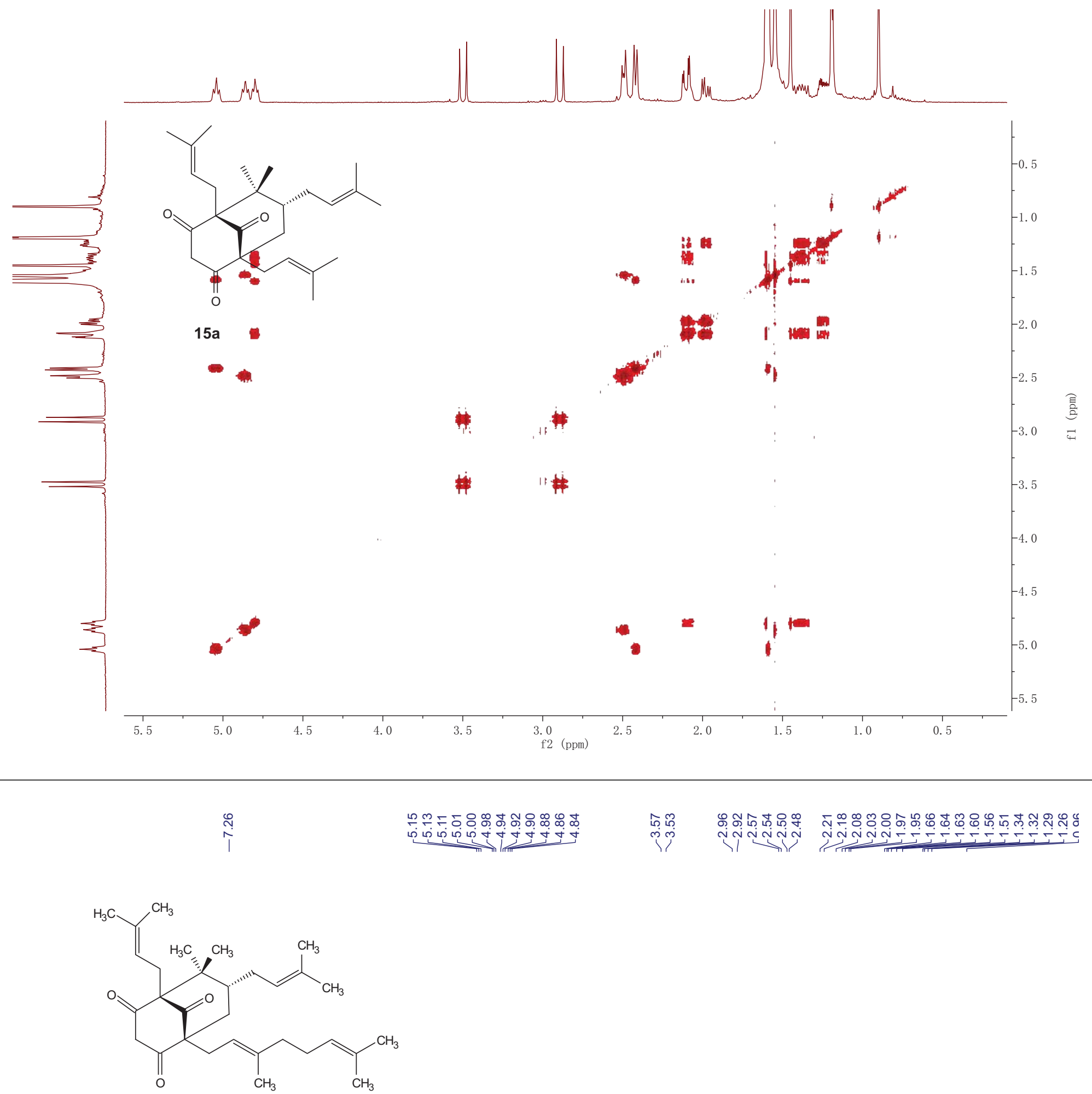

15b

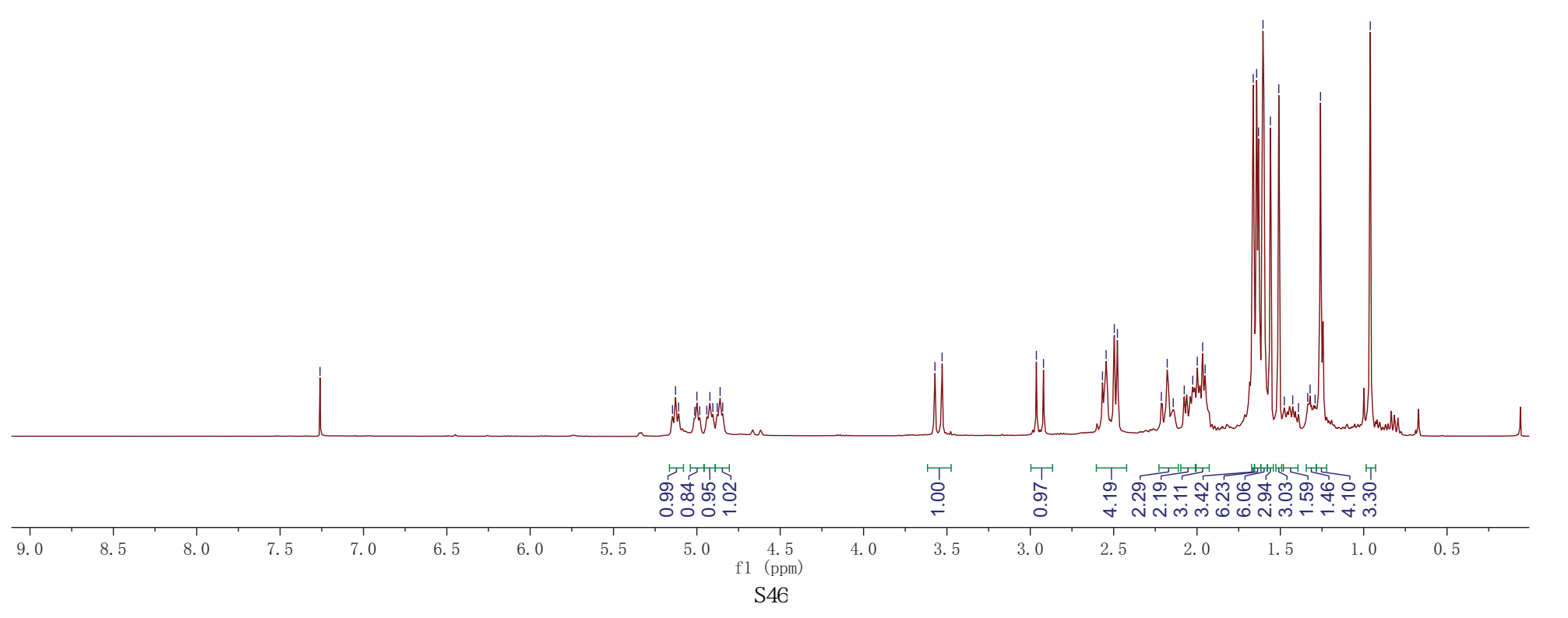


<smiles>CC(C)=CCC/C(C)=C/CC12CC[C@@H](CC=C(C)C)C(C)(C)C1(CC=C(C)C)C(=O)CC2=O</smiles>

$15 b$

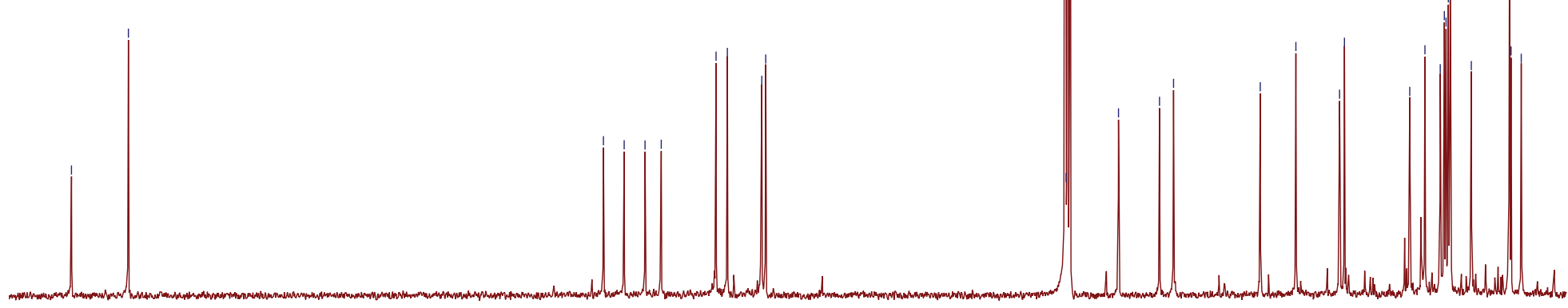

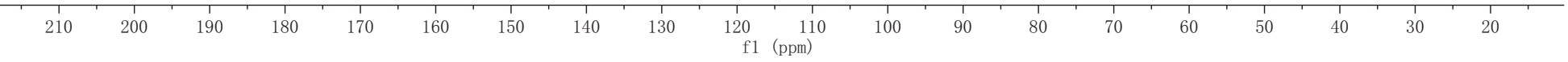

萬<smiles>COC(=O)C(CC=C(C)C)C(C)(C)C(CC=C(C)C)C(=O)OC</smiles>

$10 \mathrm{~b}$

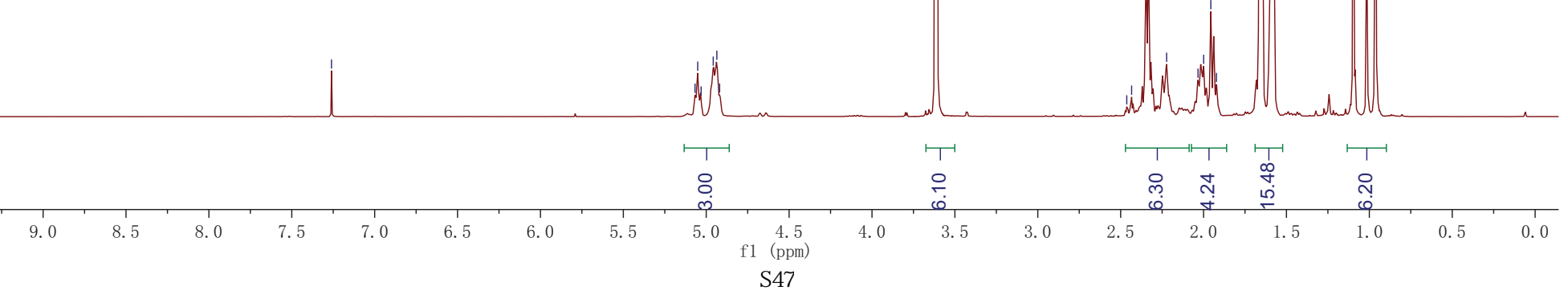


<smiles>COC(=O)C(CC=C(C)C)C(C)(C)C(CC=C(C)C)C(=O)OC</smiles>

$10 \mathrm{~b} \mathrm{cis} /$ trans $=6: 1$

only the peaks for cis-product was labelled

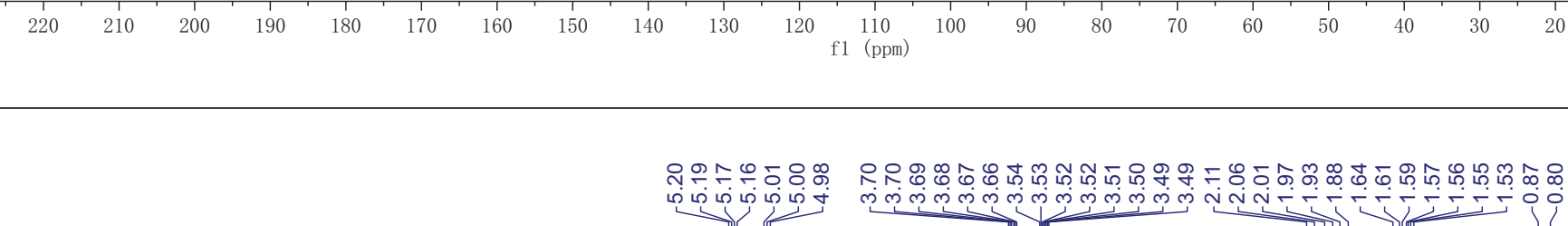<smiles>CC(C)=CCC/C(C)=C\C[C@@H](CO)C(C)(C)[C@H](CO)CC=C(C)C</smiles>

\begin{tabular}{|c|c|c|c|c|c|c|c|c|c|c|c|c|c|c|c|c|c|c|}
\hline & & & & & & & & 'T' 'T & & & 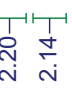 & & & 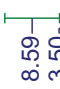 & 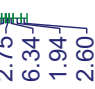 & & & \\
\hline 9.0 & 8.5 & 8.0 & 7.5 & 7.0 & 6.5 & 6.0 & 5.5 & 5.0 & $\begin{array}{c}4.5 \\
\text { f1 (ppm) }\end{array}$ & 4.0 & 3.5 & 3.0 & 2.5 & 2.0 & 1.5 & 1.0 & 0.5 & 0.0 \\
\hline
\end{tabular}


<smiles>CC(C)=CCC/C(C)=C\C[C@@H](CO)C(C)(C)C(CO)[C@H](C)C=C(C)C</smiles>

S4<smiles>CC(C)=CCC/C(C)=C\C[C@H](CO)C(C)(C)[C@H](CO)CC=C(C)C</smiles>

S4

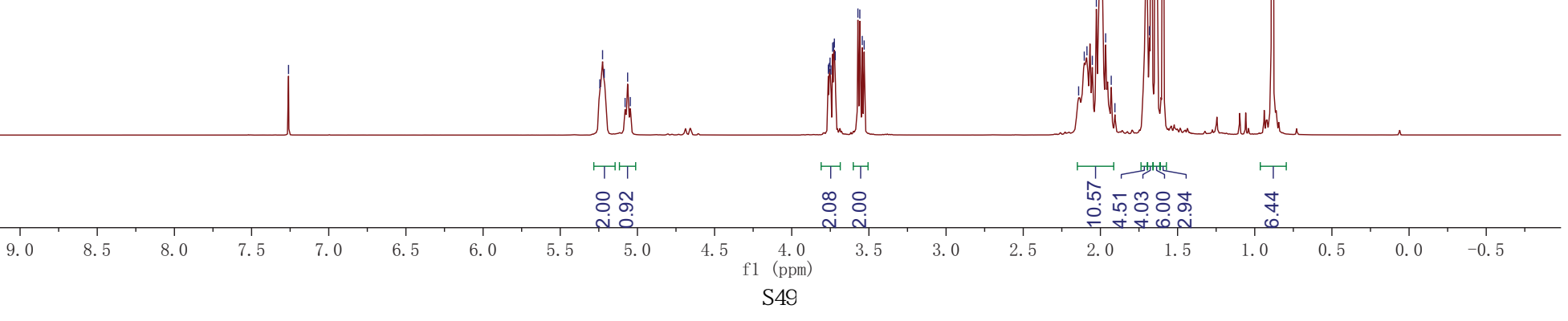


<smiles>CC(C)=CCC/C(C)=C\C[C@@H](CO)C(C)(C)[C@@H](CO)CC=C(C)C</smiles>

S4<smiles>CC(C)=CCC/C(C)=C/C[C@@H]1COC(=O)[C@H](CC=C(C)C)C1(C)C</smiles>

11b

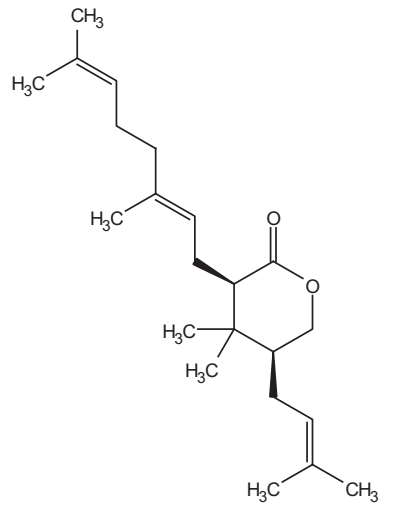

11b' 


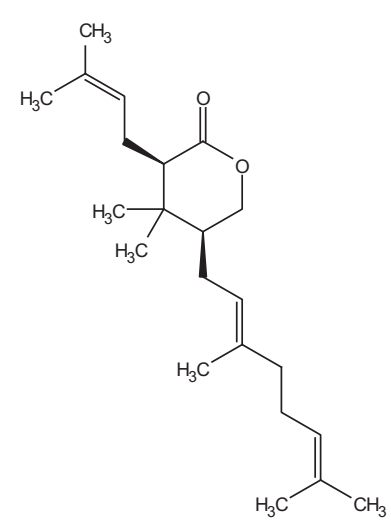

11b
站先

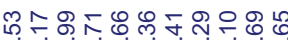

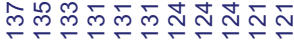

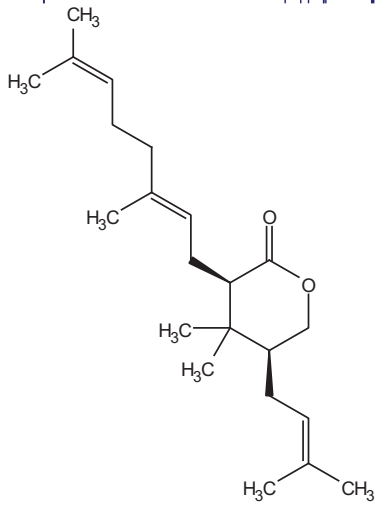

11b'

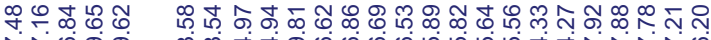

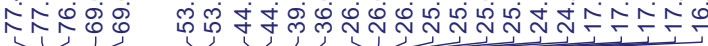

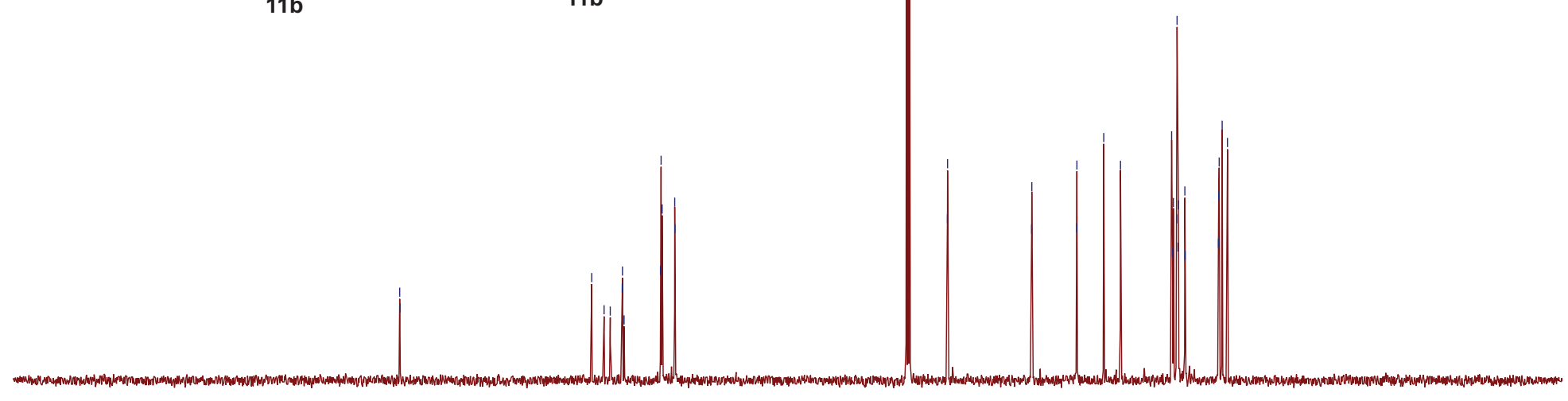

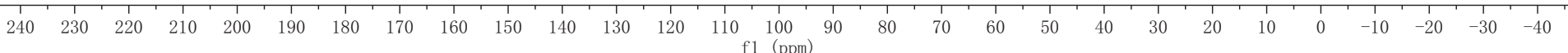

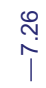

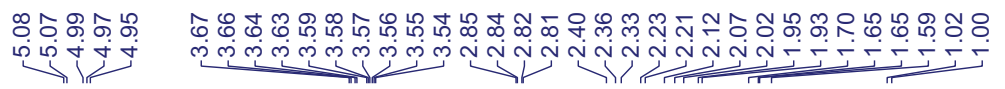<smiles>CC[C@@H](CC=C(C)CCC=C(C)C)C(C)(C)[C@@H](CC=C(C)C)C(C)=O</smiles>

$12 b$<smiles>CC(=O)[C@H](CC=C(C)CCC=C(C)C)C(C)(C)[C@@H](CCl)CC=C(C)C</smiles>

$12 b^{\prime}$ 
<smiles>CC(=O)[C@H](CC=C(C)C)C(C)(C)[C@@H](CC=C(C)C)CC=C(C)CCC=C(C)C</smiles>

$12 b$

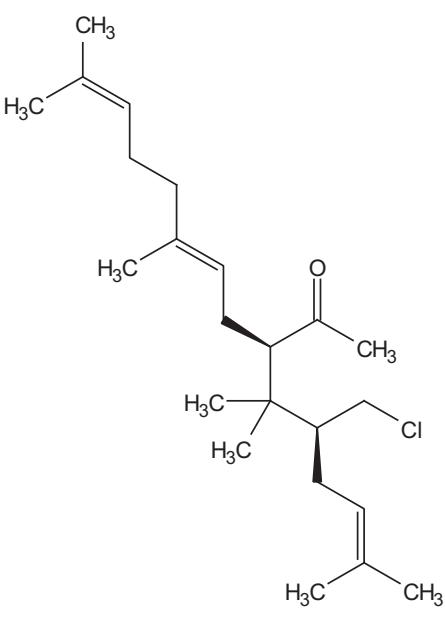

$12 b^{\prime}$<smiles>COC(=O)C(C[C@@H](CC(C)=C(C)CCC=C(C)C)C(C)(C)[C@H](CC=C(C)C)C(C)=O)C(=O)OC</smiles>

$13 b$

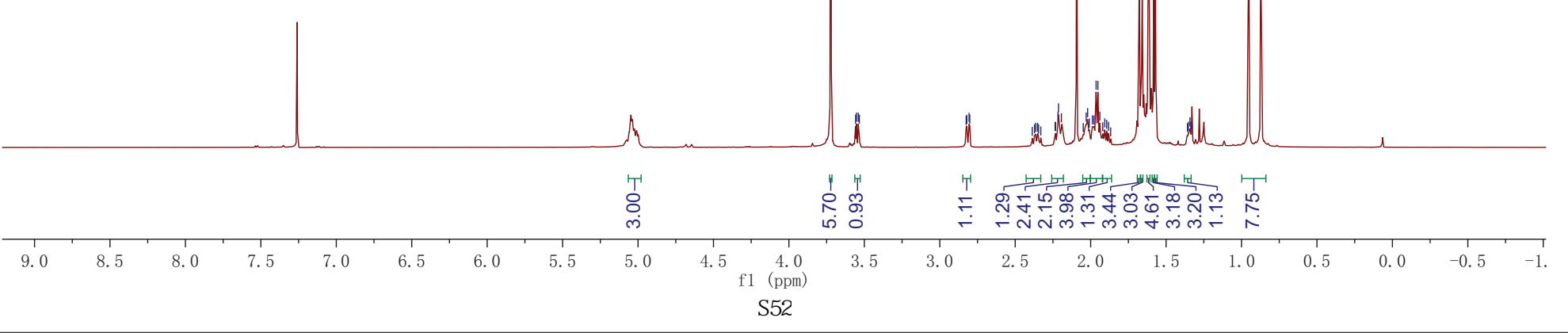


<smiles>COC(=O)C(C[C@@H](CC(C)=C(C)CCC=C(C)C)C(C)(C)[C@H](CC=C(C)C)C(C)=O)C(C)=O</smiles>

$13 b$

$\begin{array}{rrrrrrrrrrr}1 & 1 & 1 & 1 & 1 & 1 & 1 & 1 & 1 & 1 & 1 \\ \mathrm{f} 1 & 200 & 190 & 180 & 170 & 160 & 150 & 140 & 130 & 120 & 100 \\ (\mathrm{ppm})\end{array}$

ڤ人 ก மூ<smiles>COC(=O)C1CC(C(C)(C)[C@@H](C/C=C(/C)CCC=C(C)C)C(C)=O)CC(=O)C1=O</smiles><smiles>CC[C@H](COC(C)=O)C(C)(C)[C@@H](CC=C(C)C)C(C)=O</smiles>

13b' (major)<smiles>[Si]C1CC1</smiles>
in

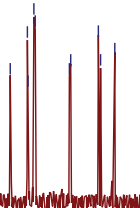

3b (minor)

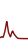


事

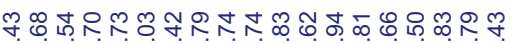

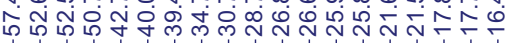<smiles>COC(=O)C1CC(C(C)(C)C(C/C=C(/C)CCC=C(C)C)C(C)=O)CC(=O)C1=O</smiles><smiles>CC=CCCC=C1CC(C(C)(C)C(CC=C(C)C)C(C)=O)CC(C(=O)OC(C)=O)C1=O</smiles>

13b' (major)

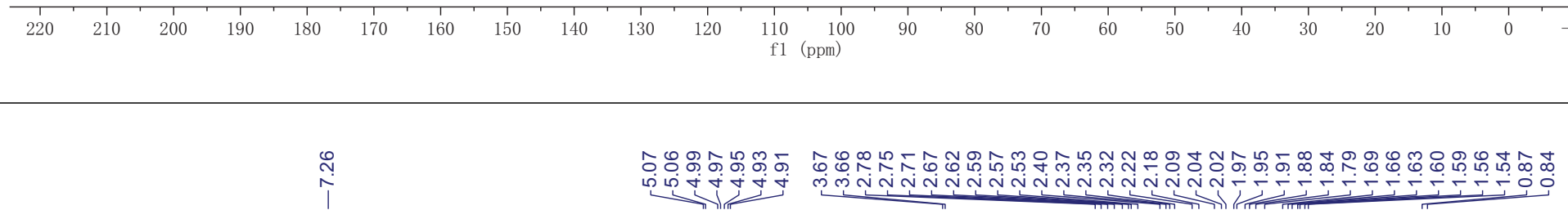<smiles>COC(=O)C(CC=C(C)C)(C[C@@H](CC=C(C)CCC=C(C)C)C(C)(C)[C@@H](CC=C(C)C)C(C)=O)C(=O)OC</smiles>

$14 c$

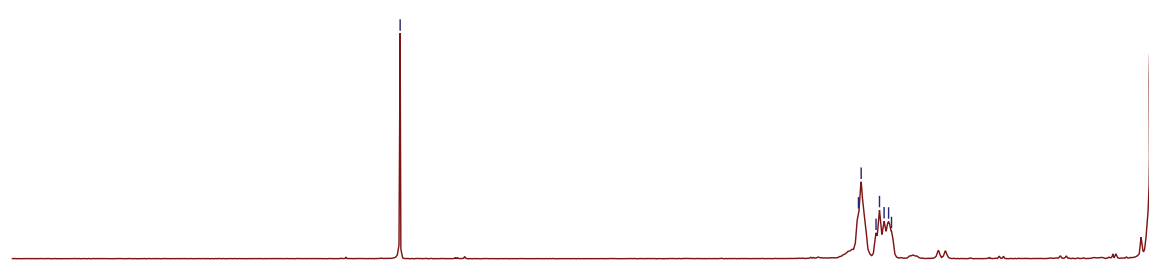

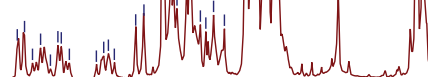

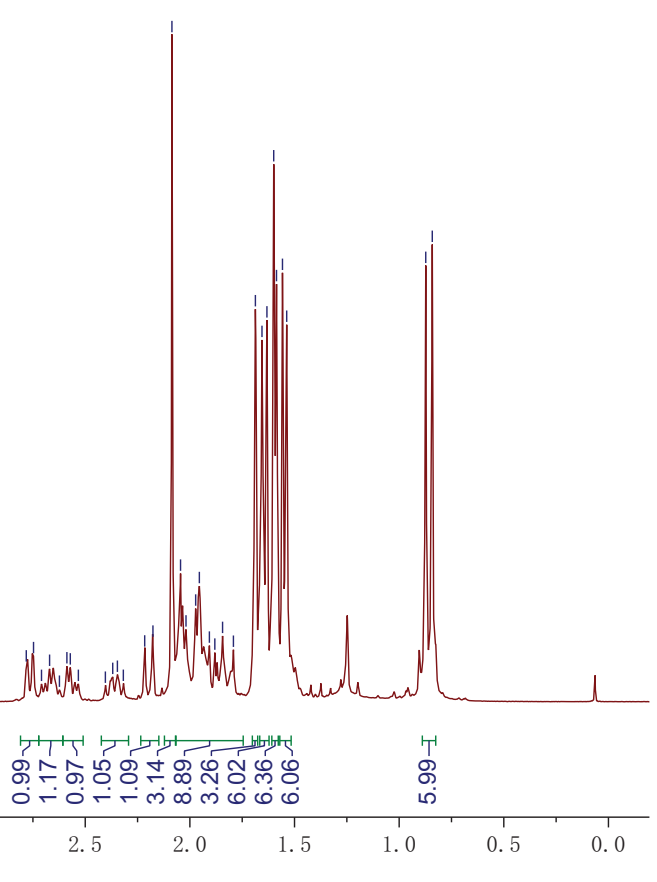




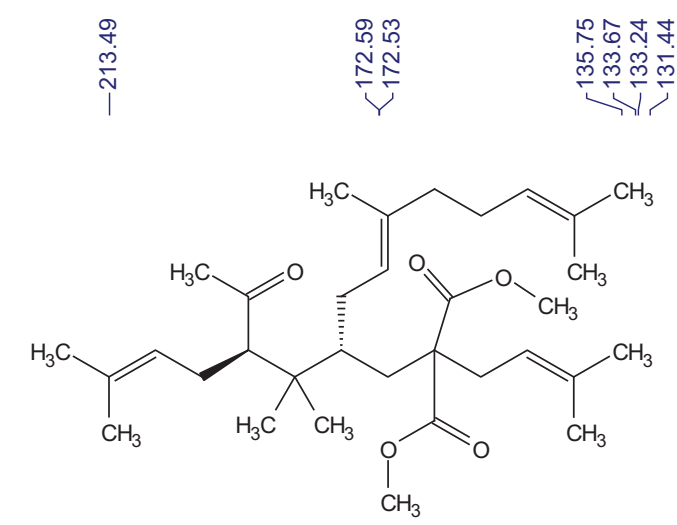

$14 c$ 
<smiles>COC(=O)C(CC=C(C)C)(C[C@@H](CC=C(C)C)C(C)(C)[C@@H](C/C=C(/C)CCC=C(C)C)C(C)=O)C(=O)OC</smiles>

$14 d$

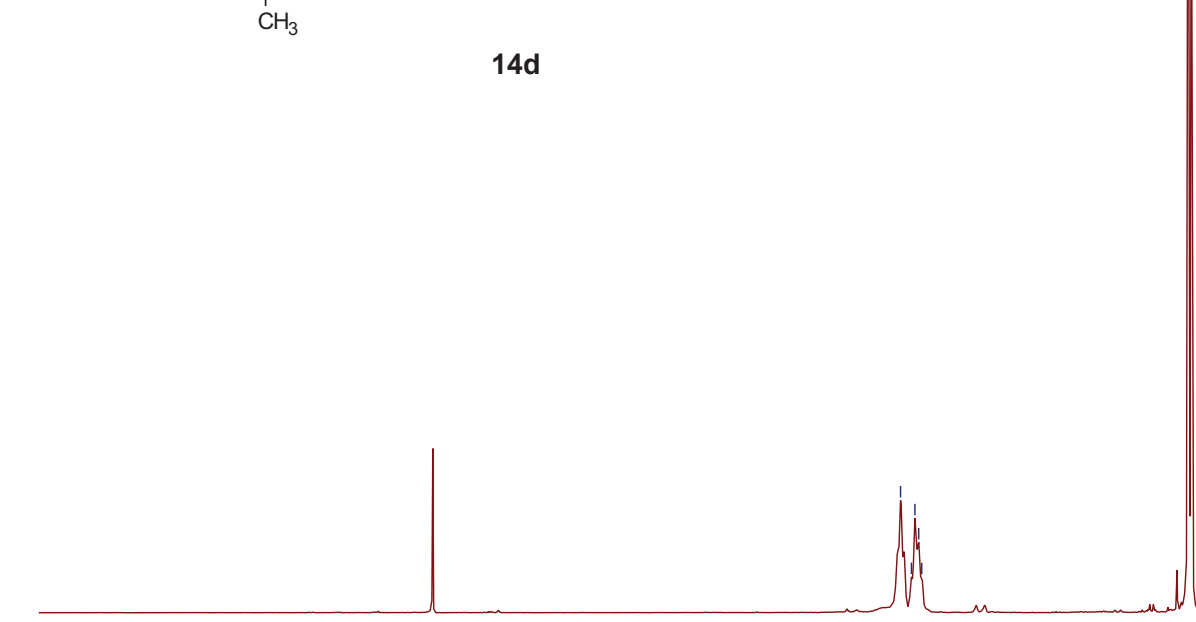

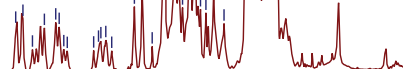

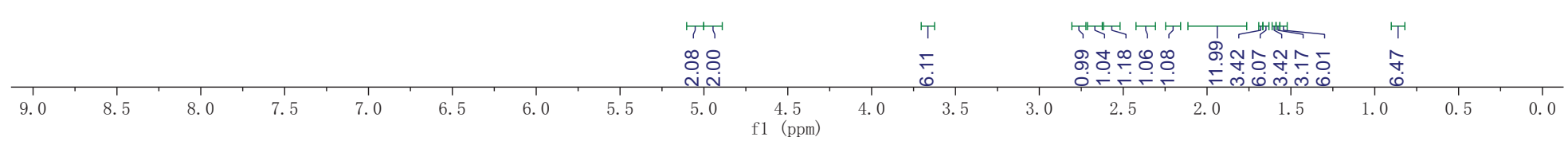<smiles>COC(=O)C(CC=C(C)C)(C[C@@H](CC=C(C)C)C(C)(C)[C@H](C/C=C(/C)CCC=C(C)C)C(C)=O)C(=O)OC</smiles>

$14 d$ 

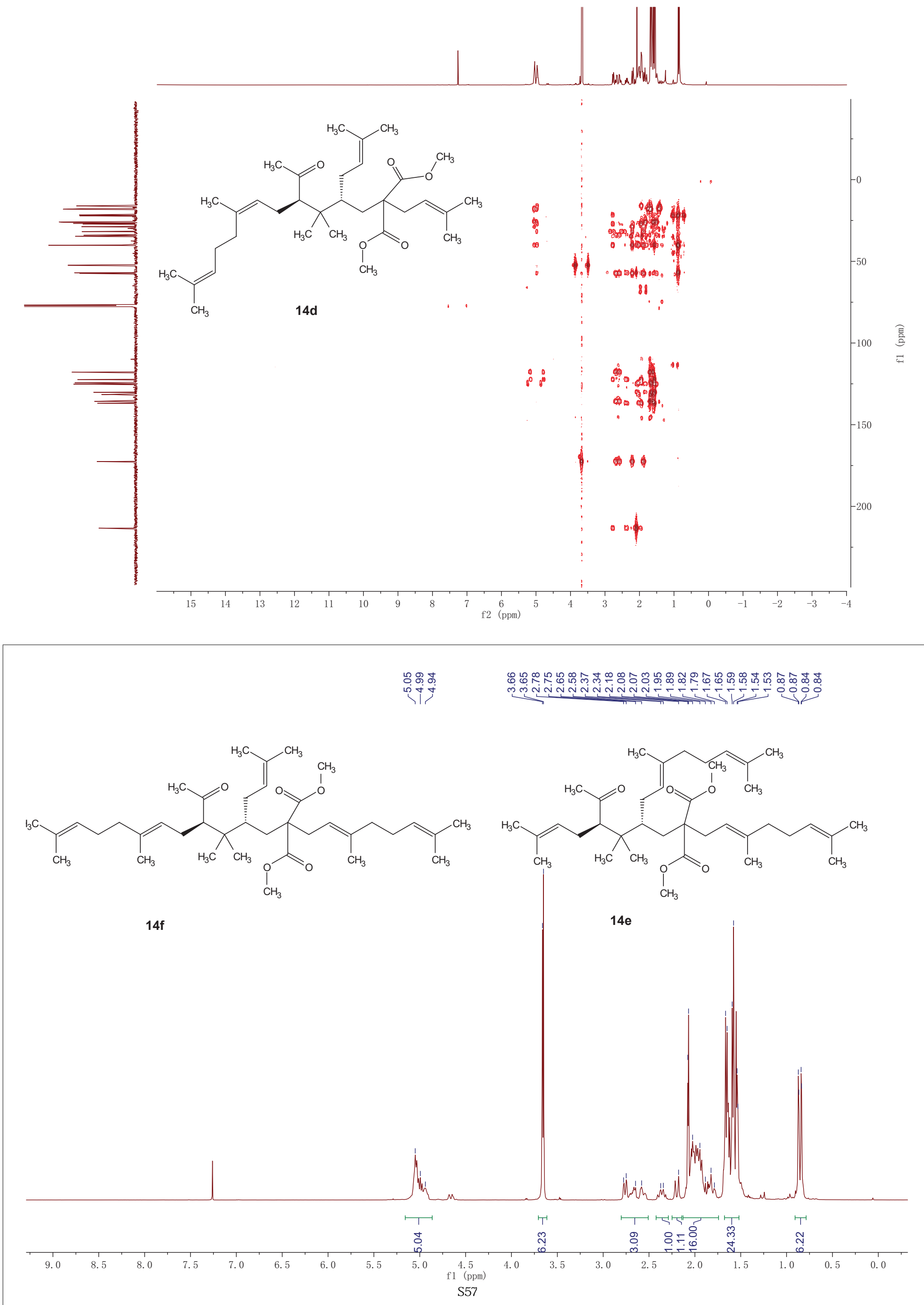


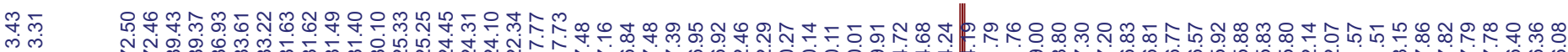
స̃<smiles>COC(=O)C(C/C=C(\C)CCC=C(C)C)(C[C@H](CC=C(C)C)C(C)(C)[C@@H](C/C=C(\C)CCC=C(C)C)C(C)=O)C(=O)OC</smiles>

$14 f$<smiles>COC(=O)C(C/C=C(\C)CCC=C(C)C)(C[C@@H](CC=C(C)CC=C(C)C)C(C)(C)[C@H](CC(C)=C(C)C)C(C)=O)C(=O)OC</smiles>

$14 \mathrm{e}$

$\sqrt{\frac{1}{4}}$<smiles>CC(C)=CCC/C(C)=C/C[C@@H]1CC2(CC=C(C)C)C(=O)CC(=O)[C@@](CC=C(C)C)(C1=O)C2(C)C</smiles>

$15 c$

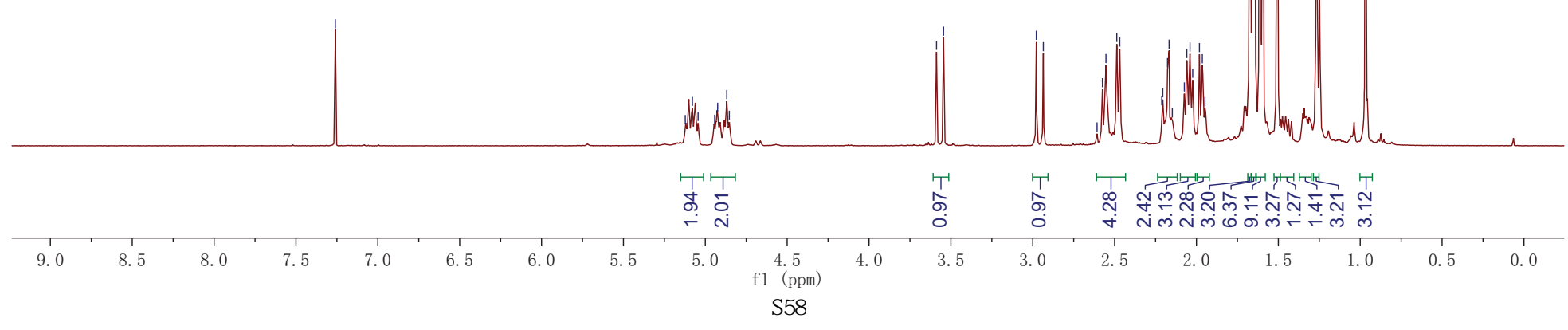




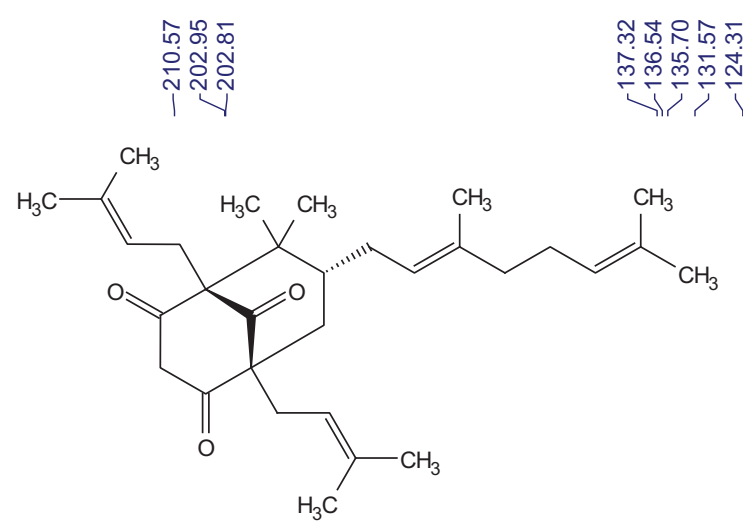

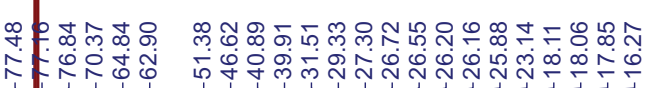

$15 c$

$\begin{array}{llllllllllllllll}240 & 230 & 220 & 210 & 200 & 190 & 180 & 170 & 160 & 150 & 140 & 130 & 120 & 110 & 100 & 90 \\ \mathrm{f} 1(\mathrm{pmm}) & \end{array}$

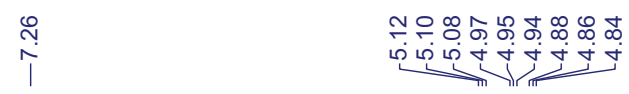

i্j

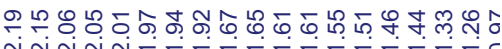

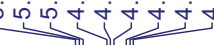

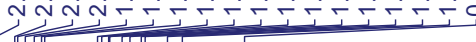
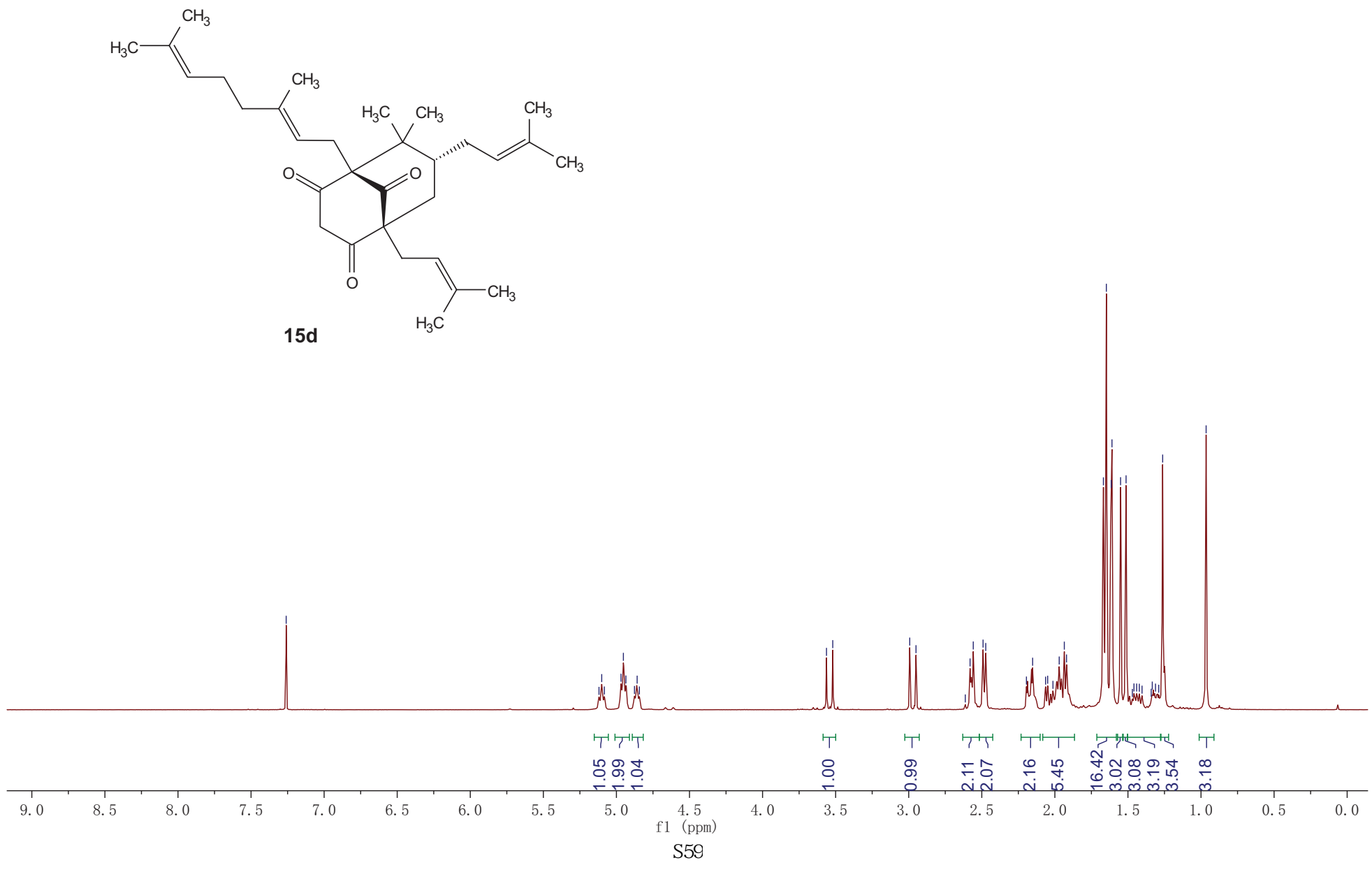


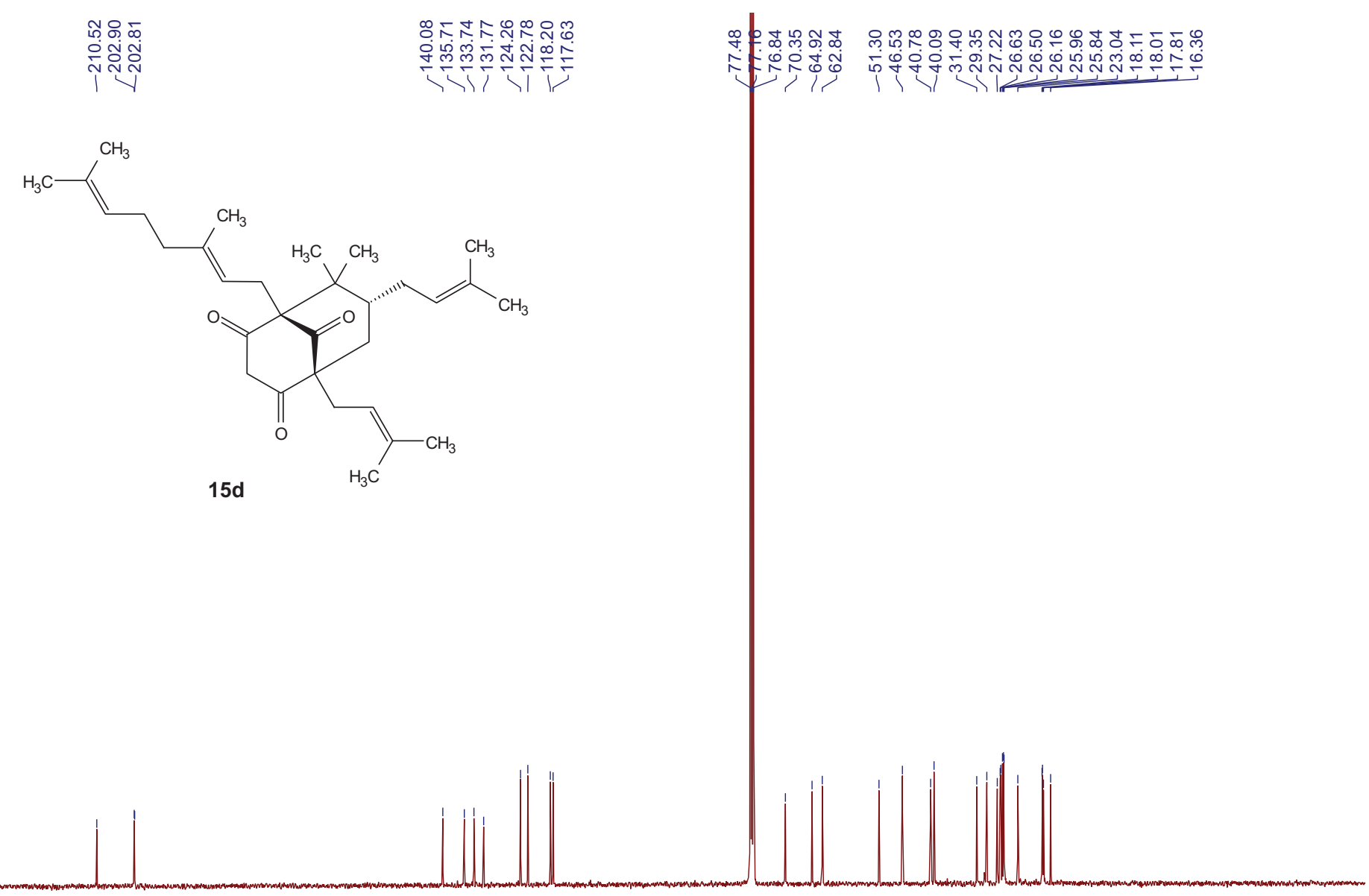

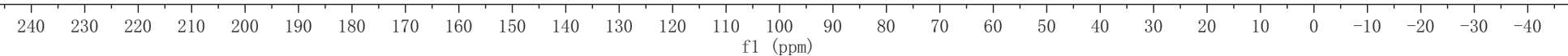

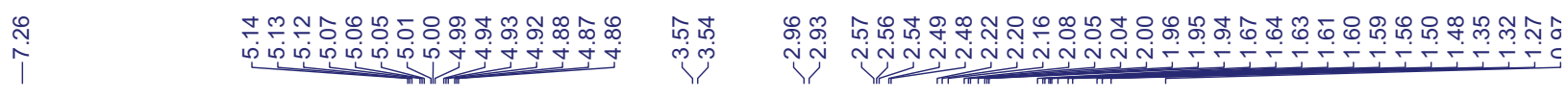<smiles>CC(C)=CCC/C(C)=C/C[C@@H]1CC2(C/C=C(\C)CCC=C(C)C)C(=O)CC(=O)[C@@](CC=C(C)C)(C1=O)C2(C)C</smiles>

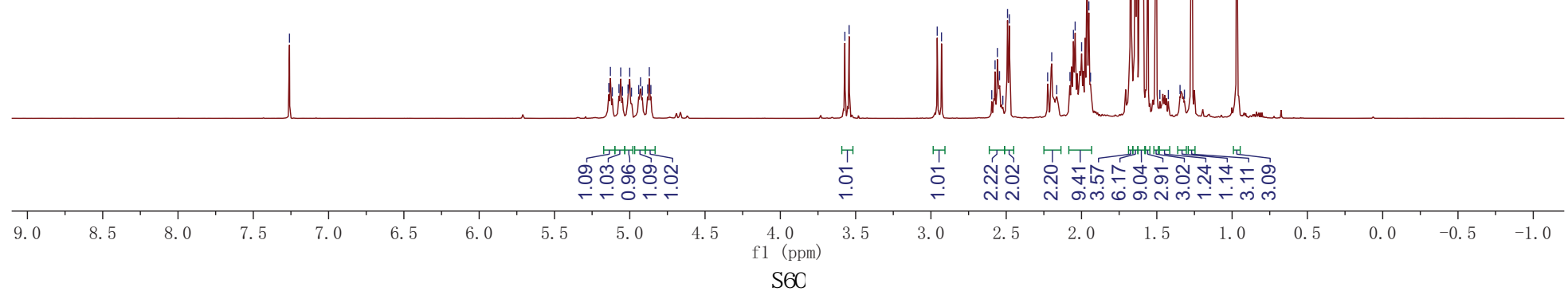



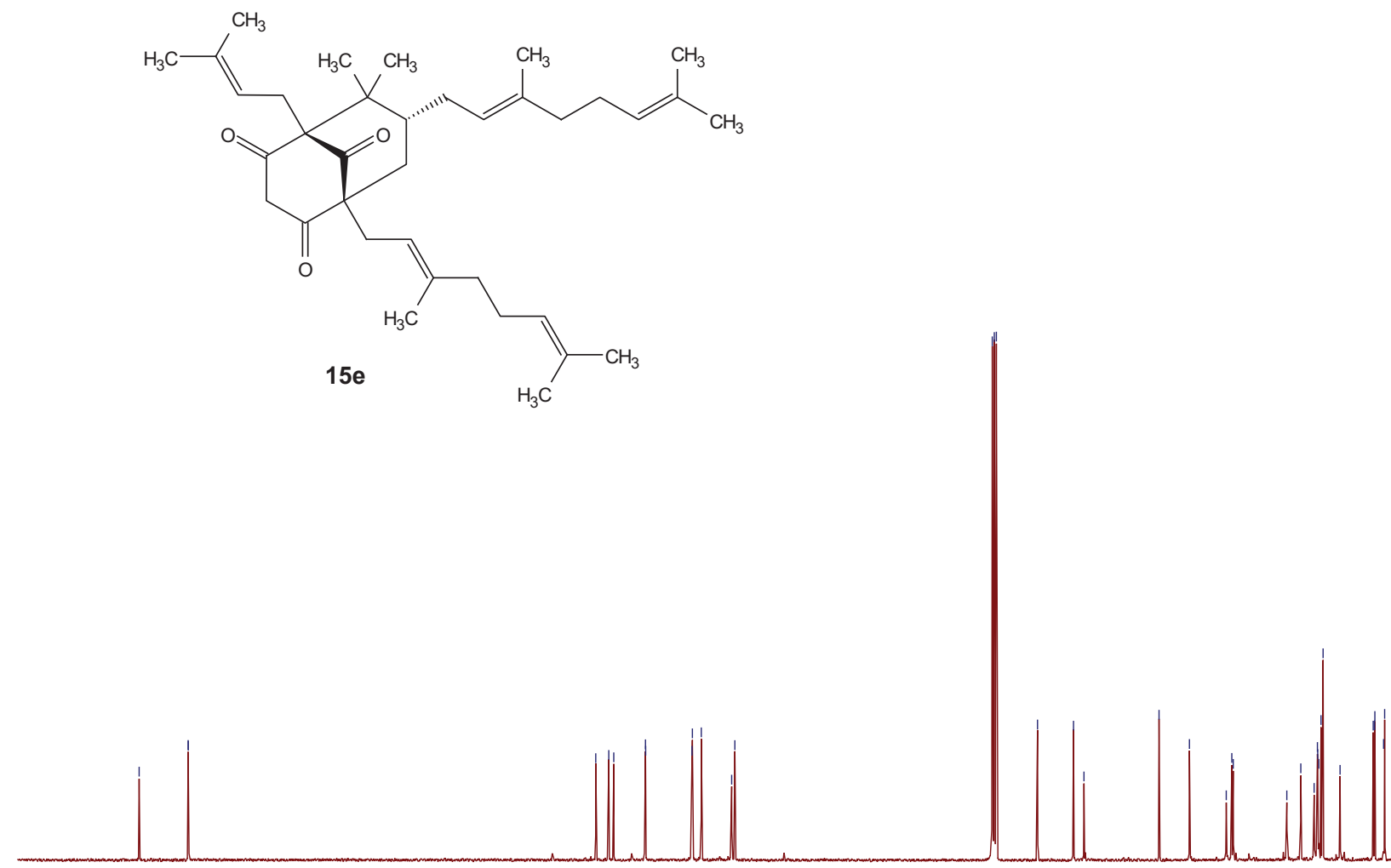

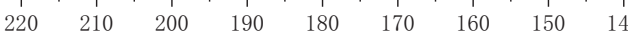

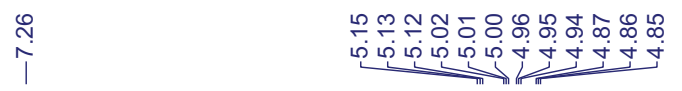
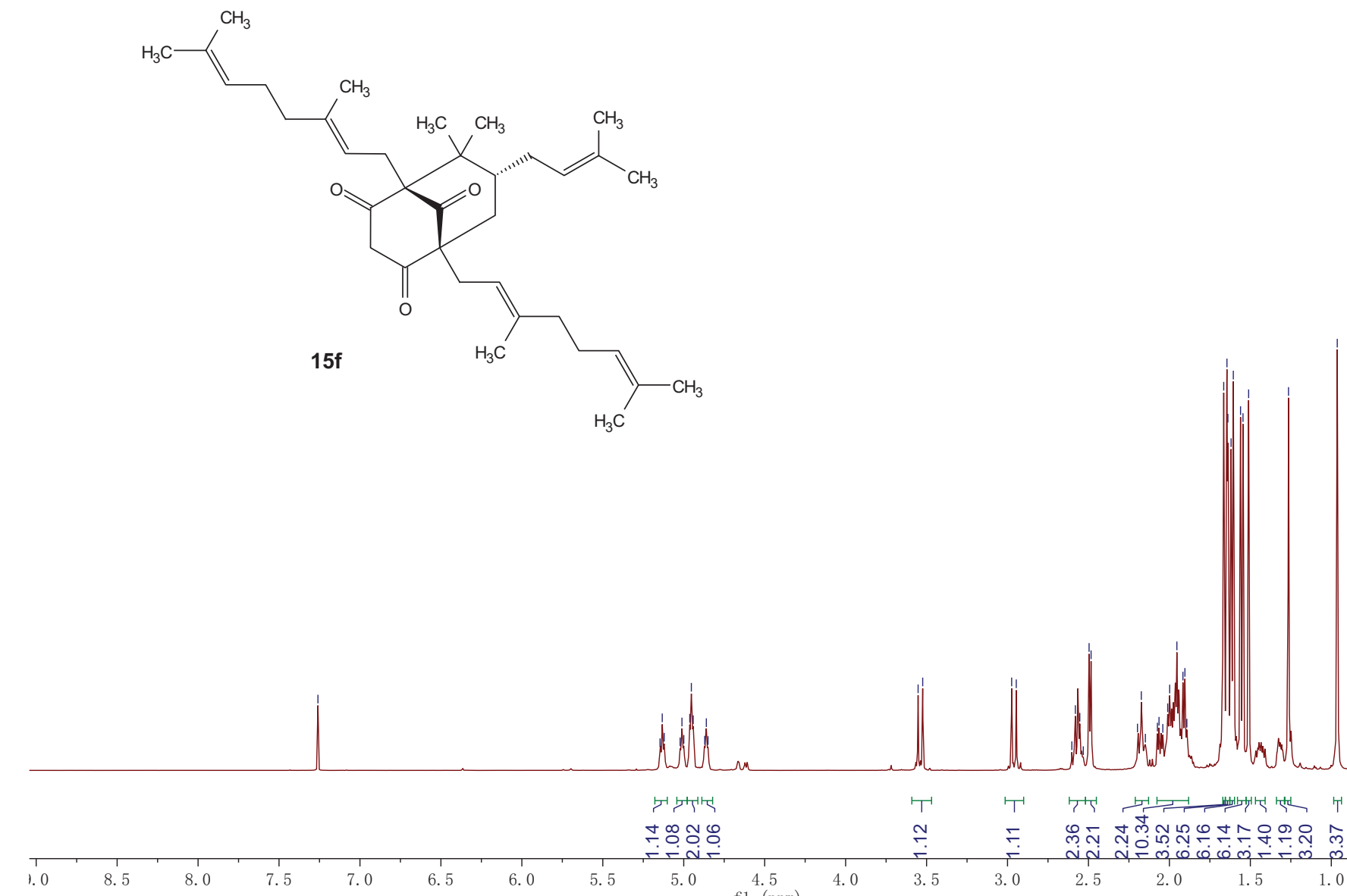

$\div \infty \mathcal{+}$ 

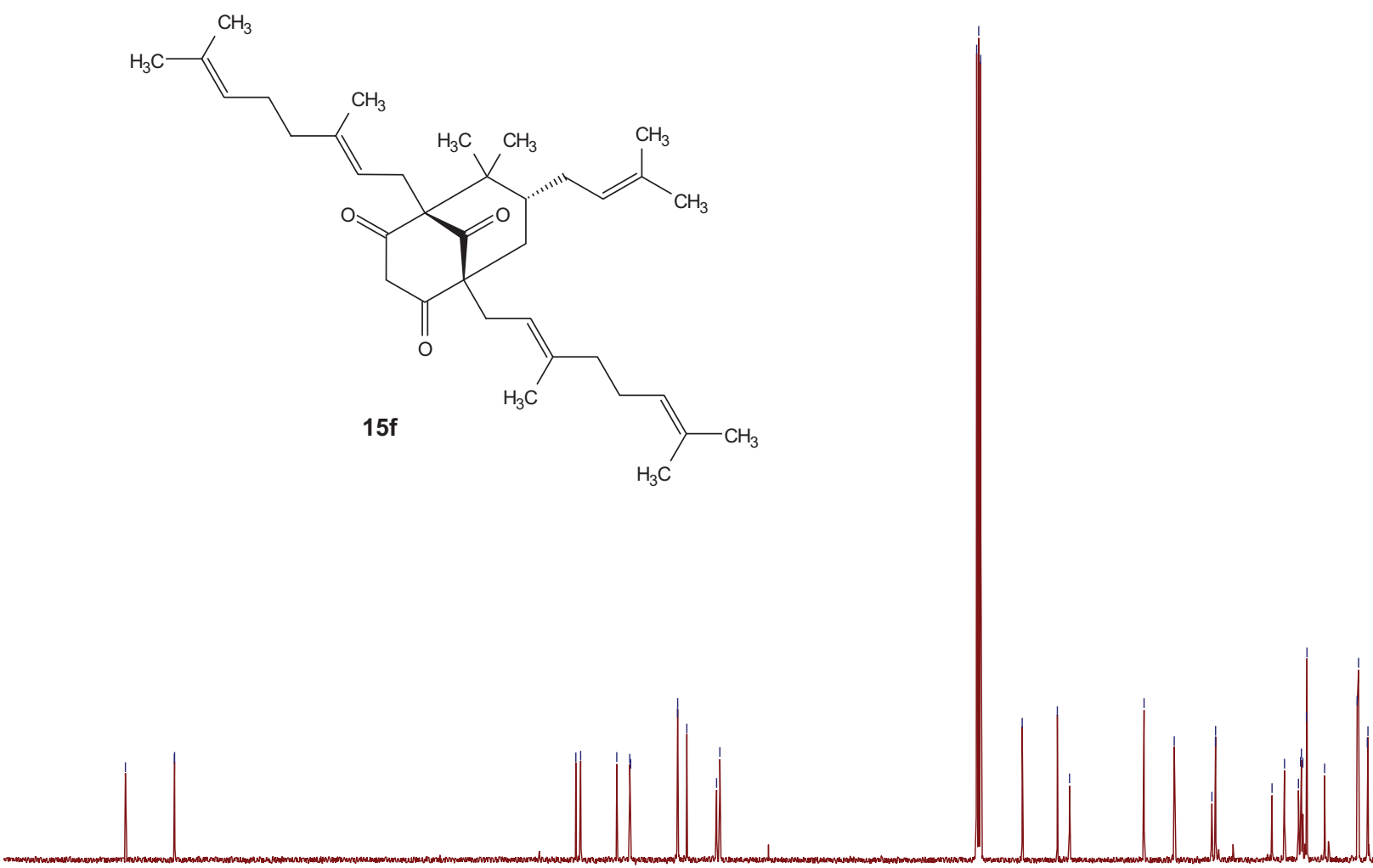

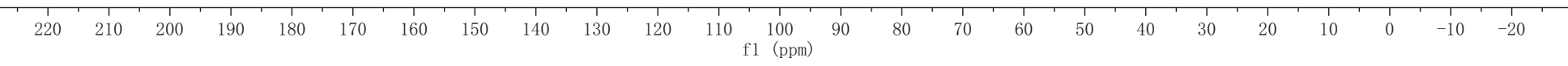




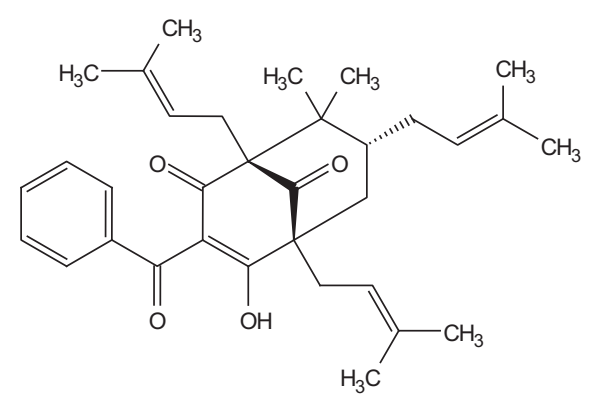

epi-clusianone (1)
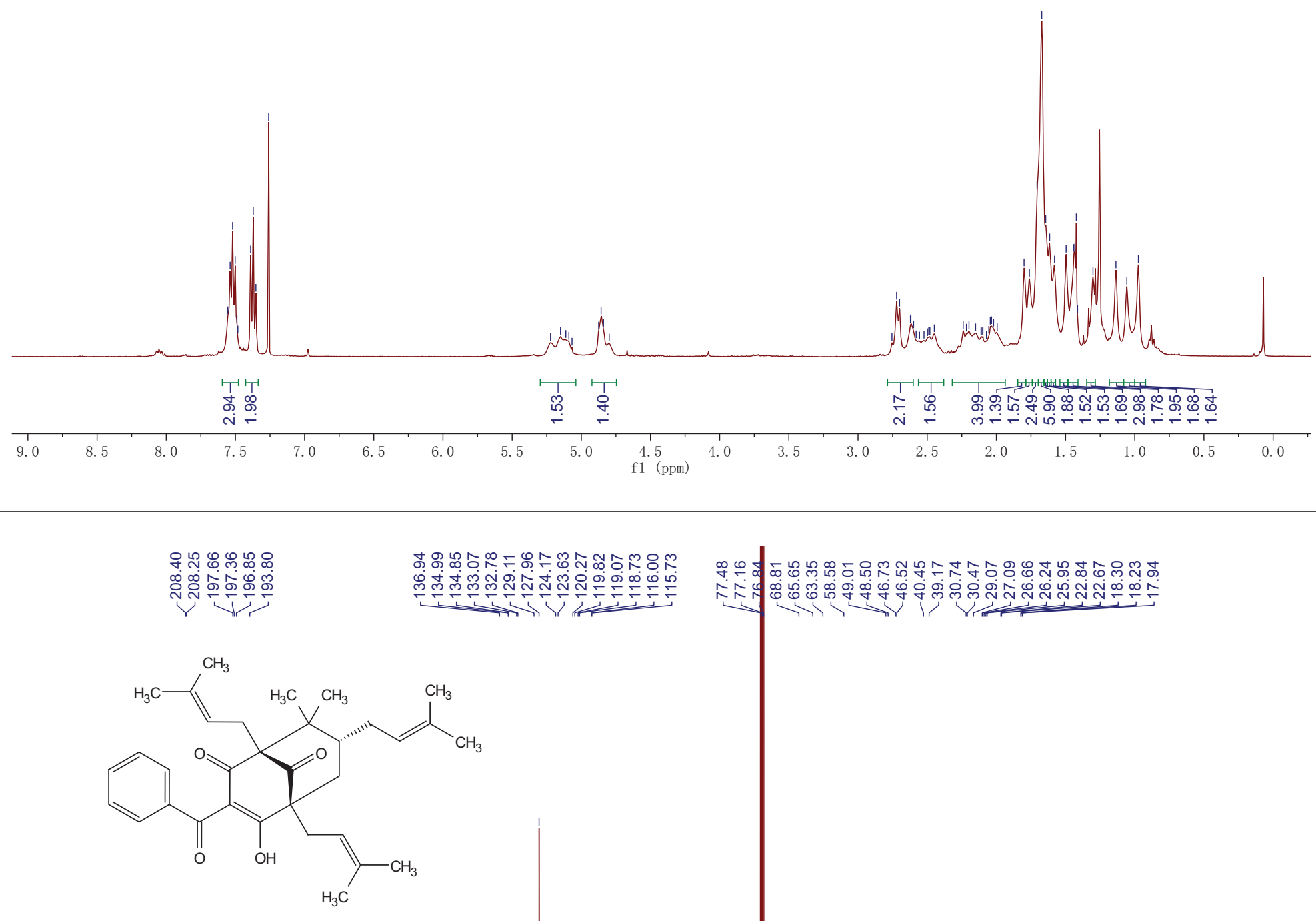

epi-clusianone (1) 
<smiles>CC(C)=CC[C@@H]1CC2(CC=C(C)C)C(=O)[C@]3(CC=C(C)C)C(=O)C(C(=O)c4ccc(O)c(O)c4)=C(O)C2(CC=C(C)C)C13C</smiles>

garcimultiflorone $\mathbf{H}$ (2)

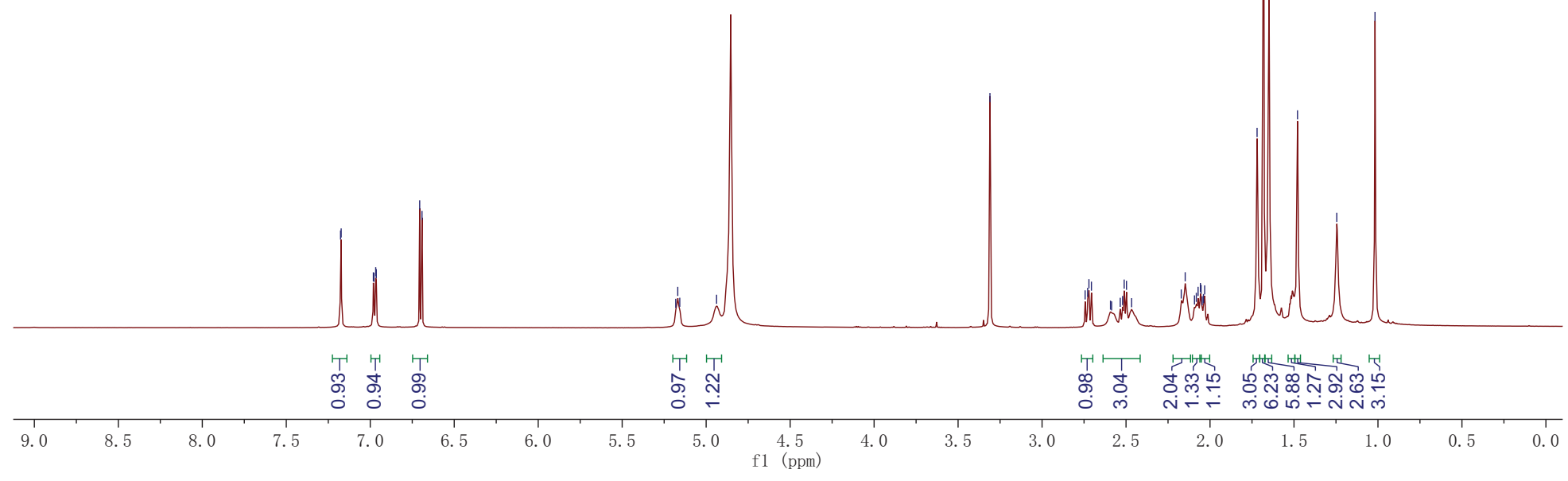

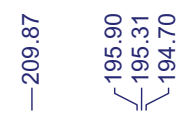

L

กิ 守

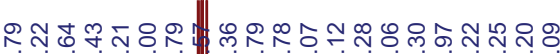

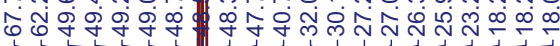<smiles>CC(C)=CC[C@@H]1C[C@@]2(CC=C(C)C)C(=O)[C@]3(CC=C(C)C)C(=O)C(C(=O)c4ccc(O)c(O)c4)=C(O)C1(C)C23C</smiles>

garcimultiflorone H (2) 
๕)<smiles>CC(C)=CC[C@@H]1C[C@@]2(CC=C(C)C)C(=O)c3c(oc4c(O)c(O)ccc4c3=O)C1(CC=C(C)C)C(C)(C)[C@@H]2O</smiles>

regio-garcimultiflorone I (21)

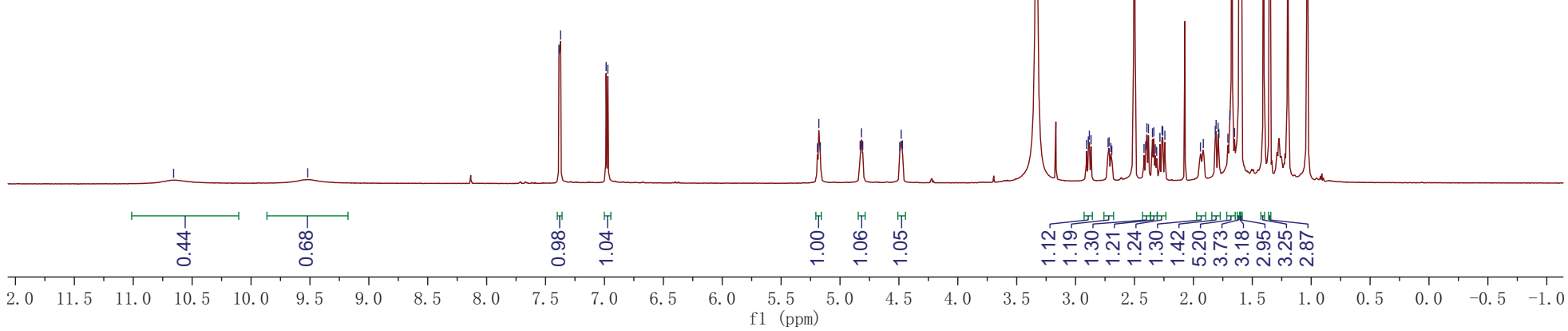

a. एँ<smiles>CC(C)=CC[C@@H]1CC2(CC=C(C)C)C(=O)c3c(oc4c(O)c(O)ccc4c3=O)[C@](CC=C(C)C)(C1=O)C2(C)C</smiles>

regio-garcimultiflorone I (21) 


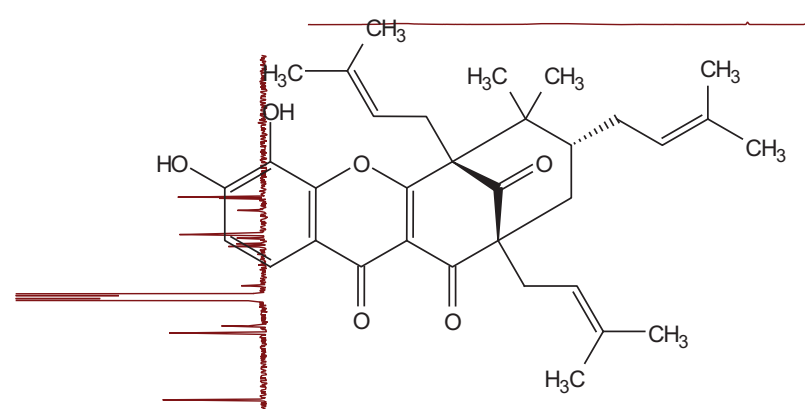

egio-garcimultiflorone I (21)
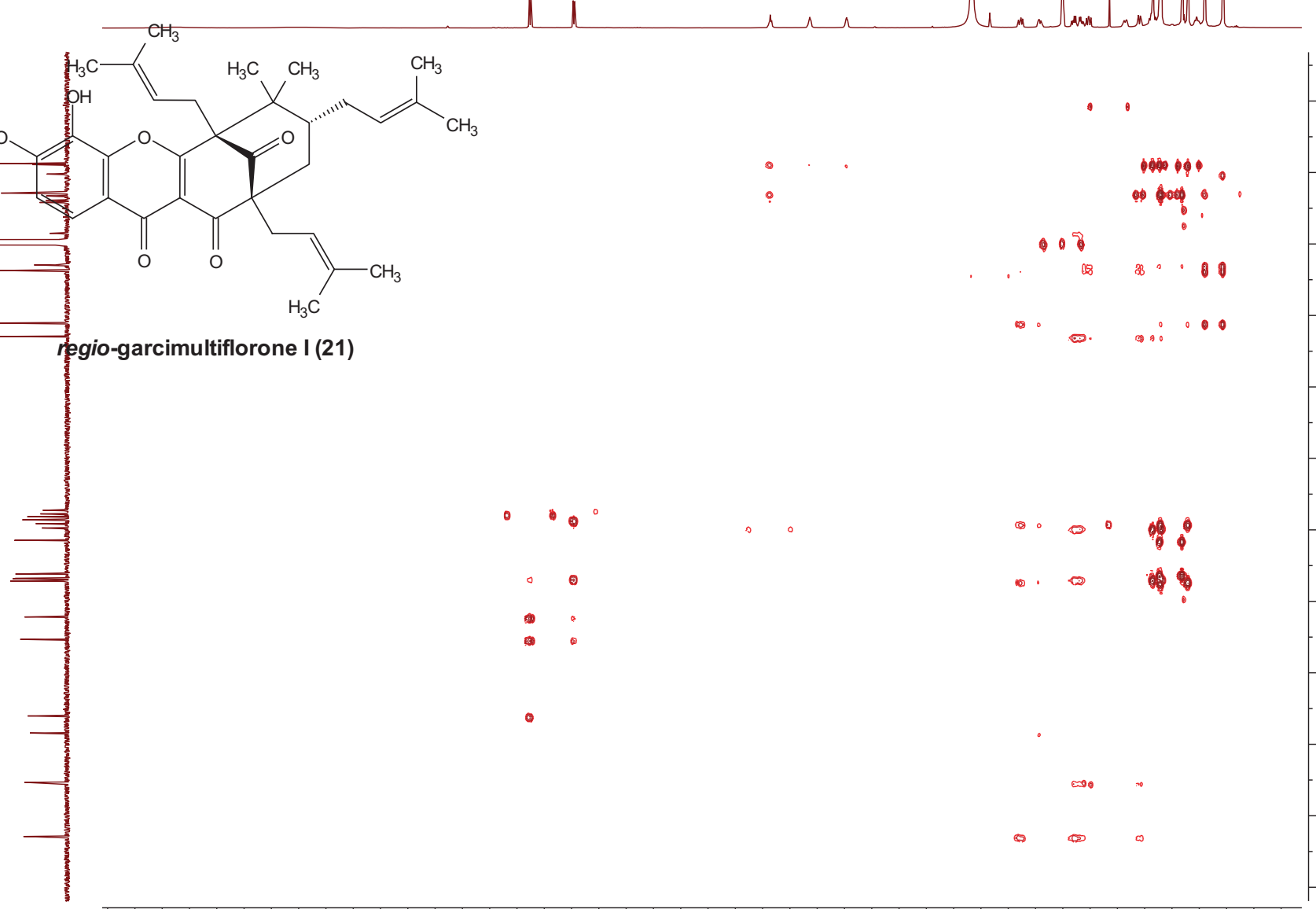

-0
-20
-40
-60
-80
$-100 \quad$ 형
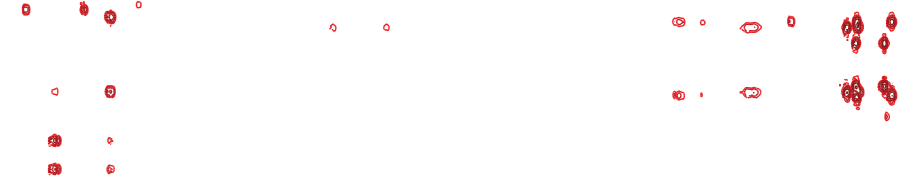<smiles>CC(C)=CC[C@@H]1CC2(CC=C(C)C)C(=O)c3c(oc4cc(O)c(O)cc4c3=O)[C@](CC=C(C)C)(C1=O)C2(C)C</smiles>

garcimultiflorones I (3)

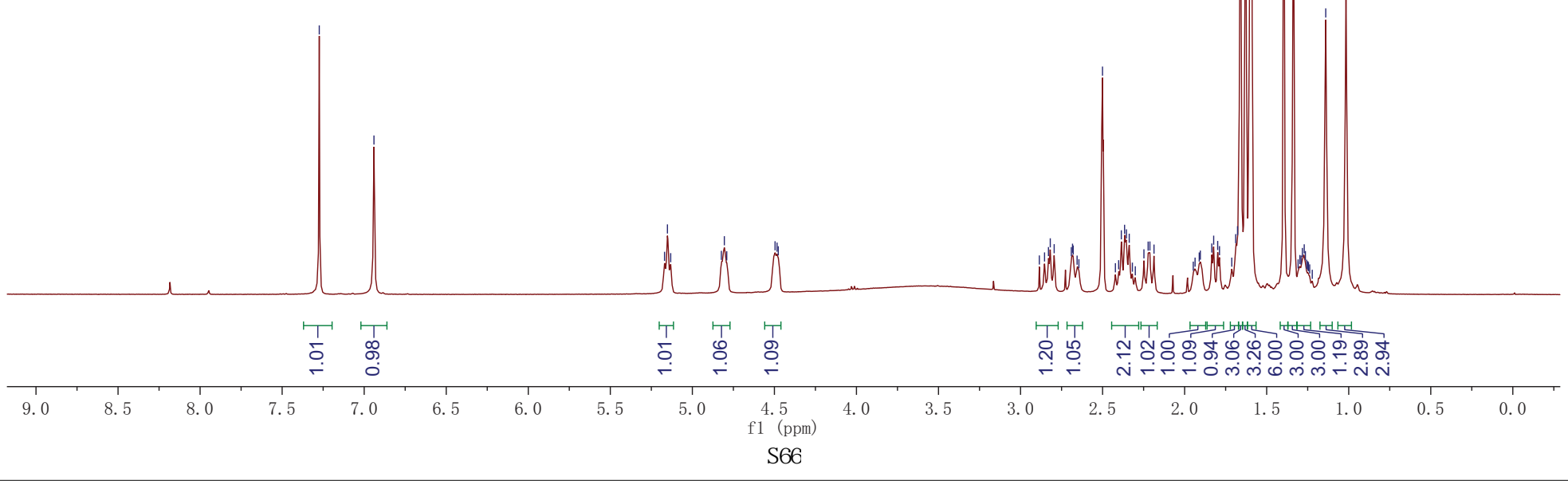


<smiles>CC(C)=CC[C@@H]1C[C@@]2(CC=C(C)C)C(=O)c3c(oc4cc(O)c(O)cc4c3=O)C1(CC=C(C)C)C(C)(C)[C@H]2O</smiles>

garcimultiflorones I (3)

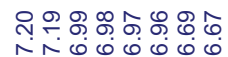

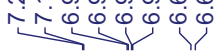

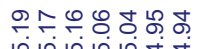

مि<smiles>CC(C)=CCC/C(C)=C/C[C@@]12C[C@H](CC=C(C)C)C(C)(C)[C@@](CC=C(C)C)(C1=O)C(O)=C(C(=O)c1ccc(O)c(O)c1)C2=O</smiles>

guttiferone I (4)

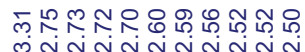

minnNinnNN

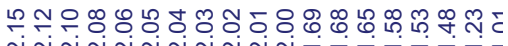

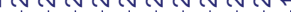
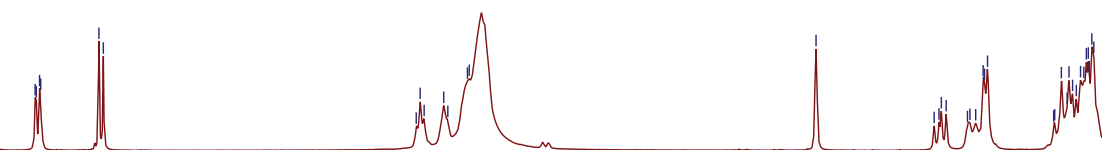

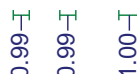

\section{कृष}

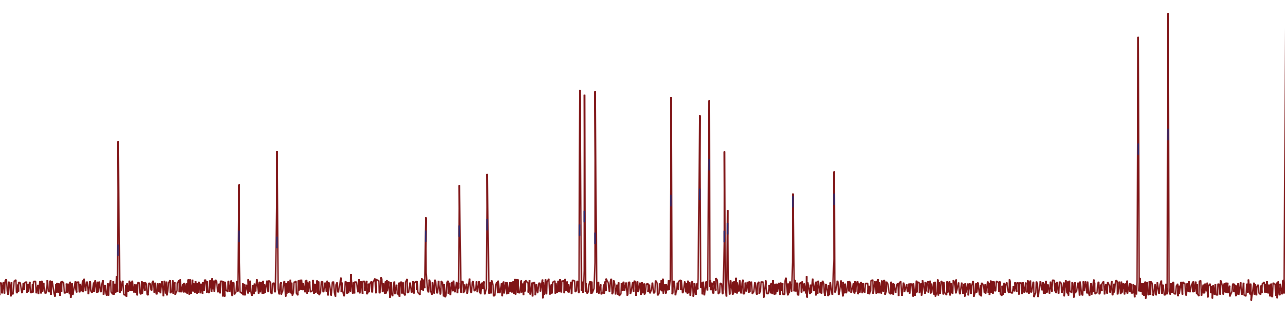


<smiles>CC(C)=CCC/C(C)=C/CC12C[C@H](CC=C(C)C)C(C)(C)C(CC=C(C)C)(C1=O)C(O)=C(C(=O)c1ccc(O)c(O)c1)C2=O</smiles>

guttiferone I (4)

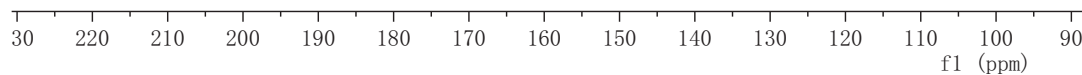<smiles>CC=CC[C@H]1[C@H](C/C=C(\C)CCC=C(C)C)CC2(CC=C(C)C)C(=O)C(CC=C(C)C)(C(=O)C(C(=O)c3ccc(O)c(O)c3)=C2O)C1(C)C</smiles>

oblongifolin A (5)

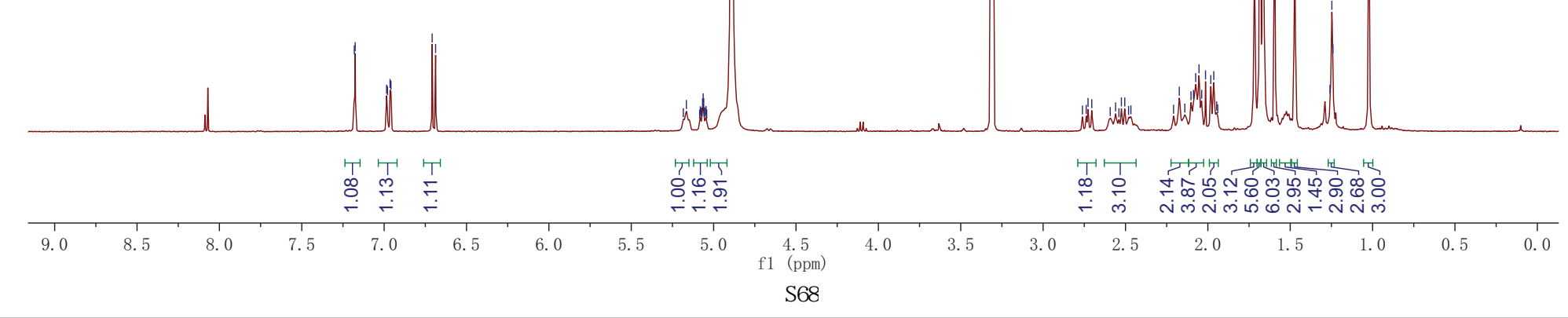


<smiles>CC(C)=CCC/C(C)=C/C[C@H]1CC2(CC=C(C)C)C(=O)C(CC=C(C)C)(C1=O)C(C)(C)C(C(=O)c1ccc(O)c(O)c1)=C2O</smiles>

oblongifolin A (5)
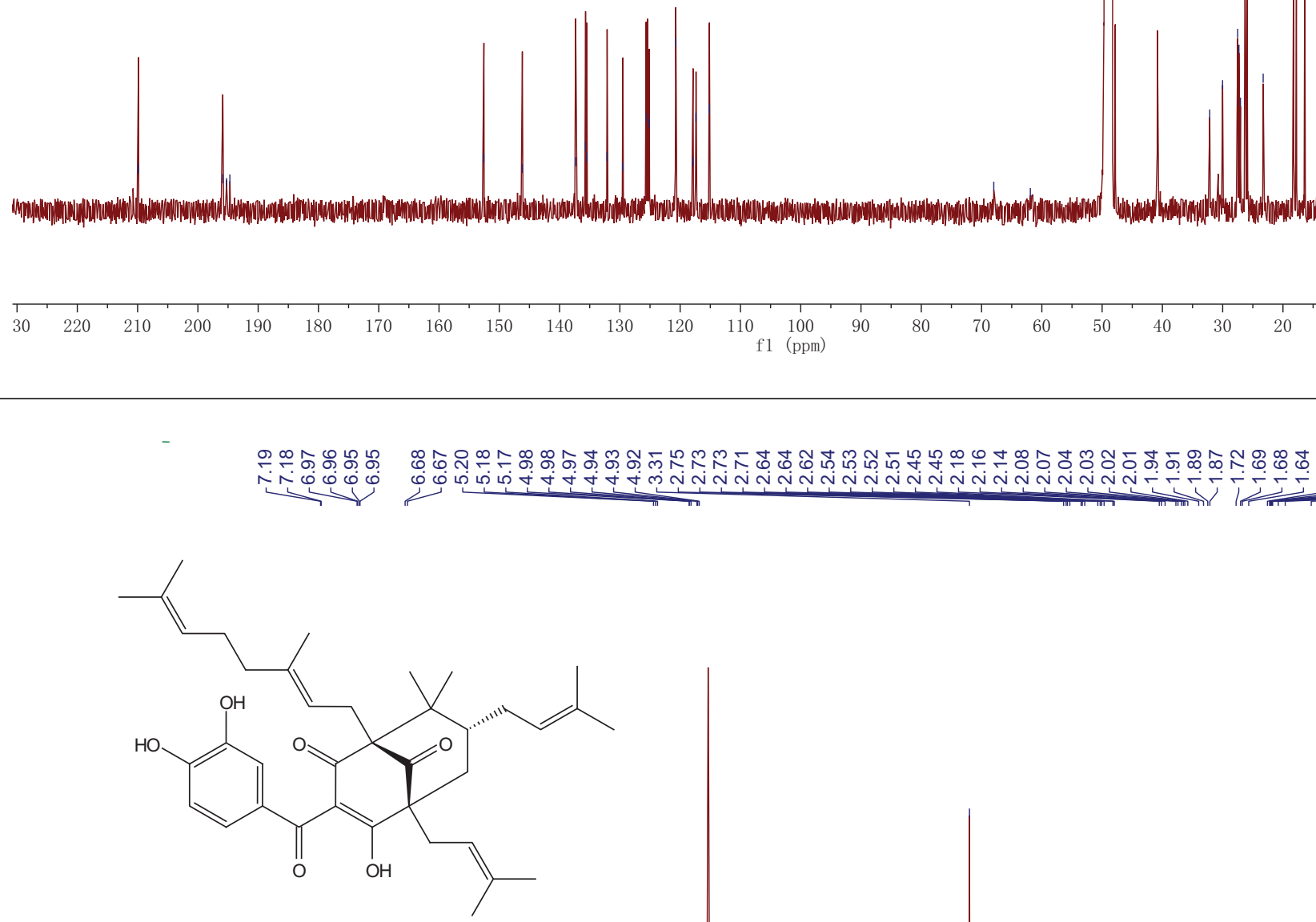

regio-guttiferone I (6)
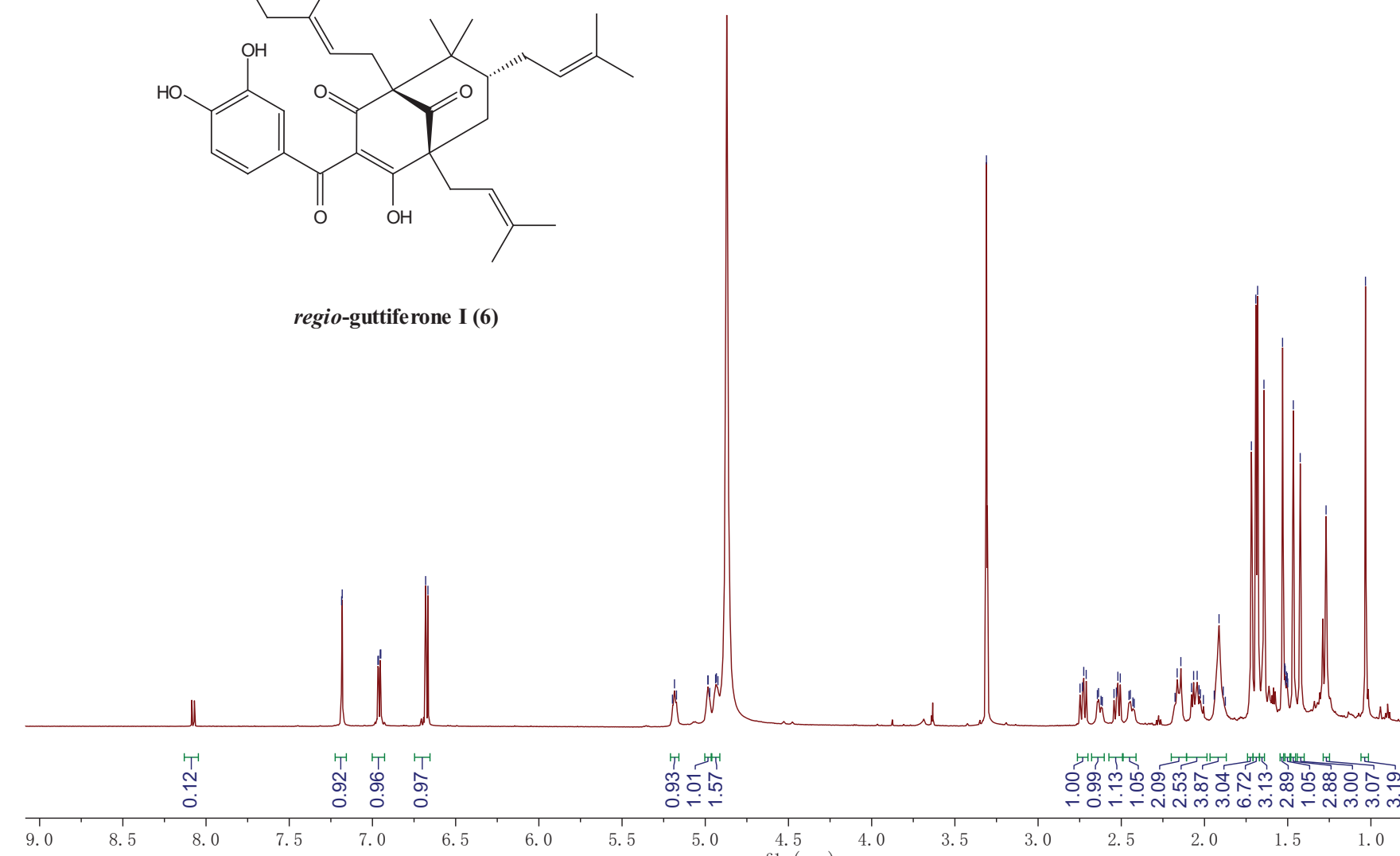

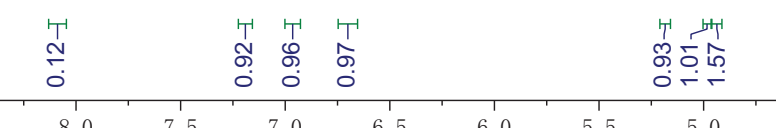




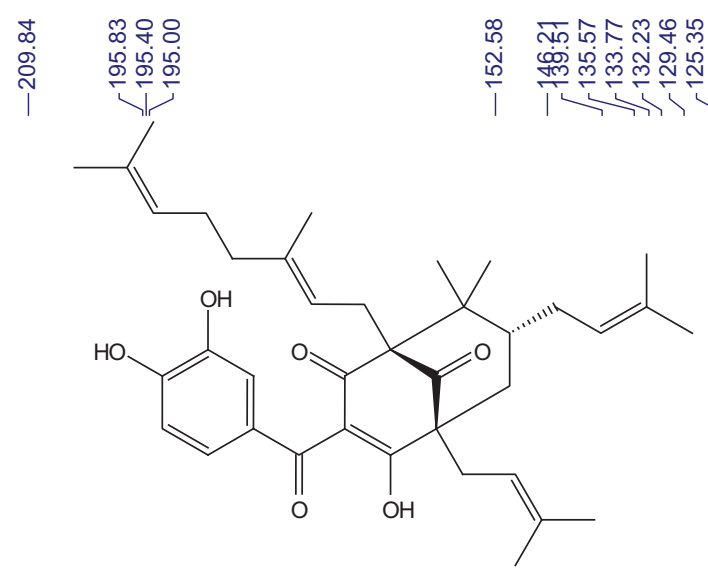

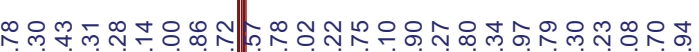

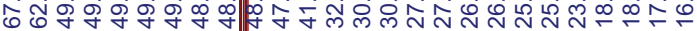

regio-guttiferone I (6)

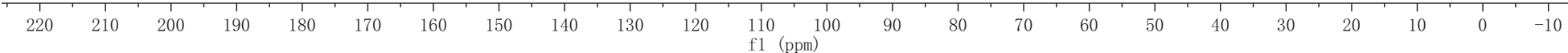

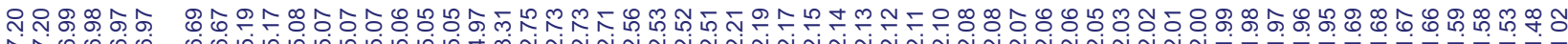
Л

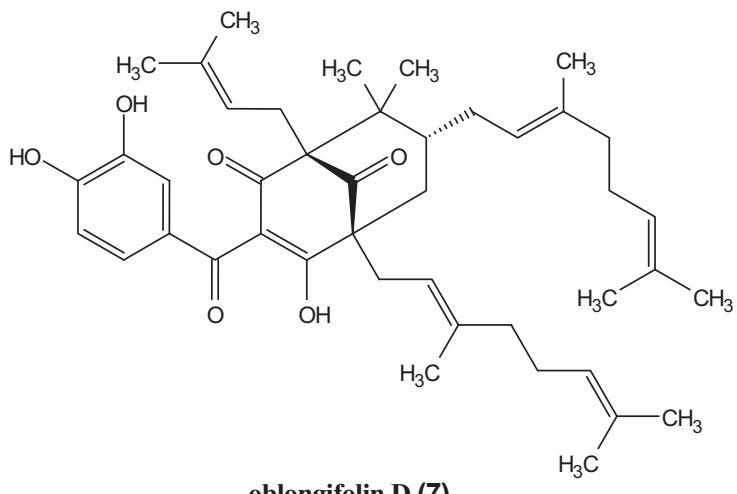

oblongifolin D (7)

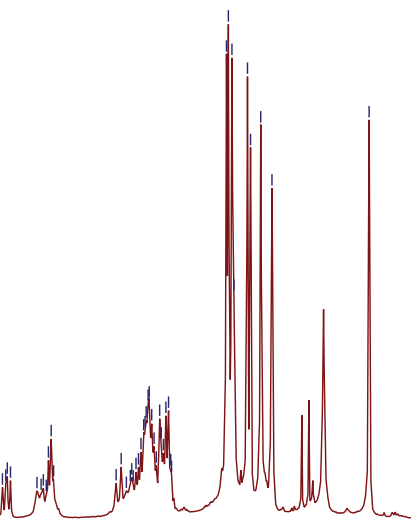

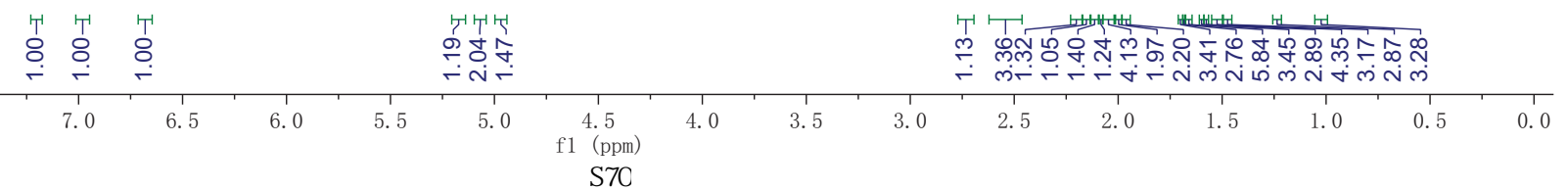


<smiles>CC(C)=CCC/C(C)=C/C[C@@H]1C[C@]2(C/C=C(\C)CCC=C(C)C)C[C@]13C(C)(C)C(O)=C(C(=O)c1ccc(O)c(O)c1)C(=O)[C@@]3(CC=C(C)C)C2=O</smiles>

blongifolin D (7)

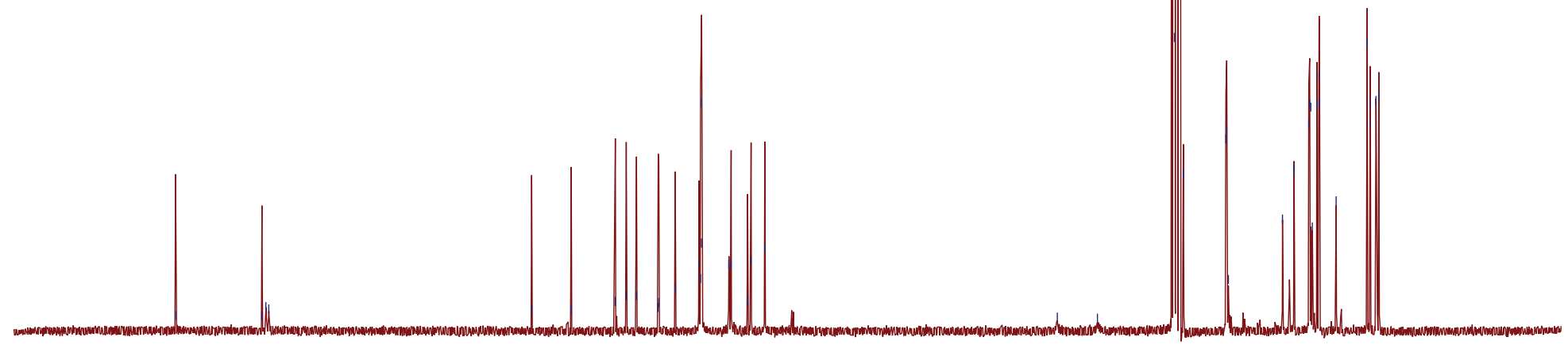

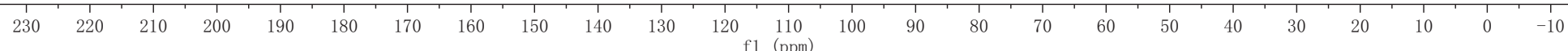

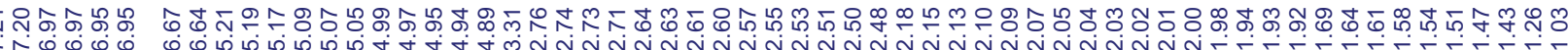

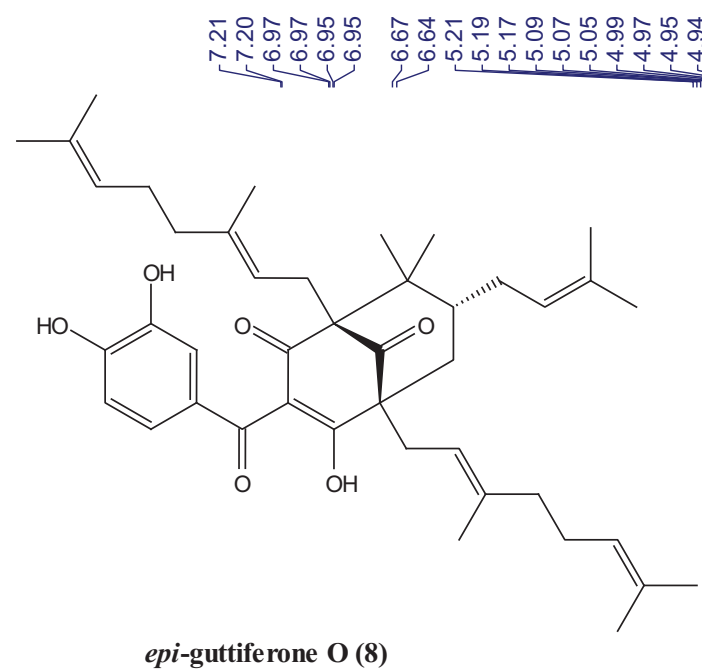

epi-guttiferone $\mathbf{O}(8)$

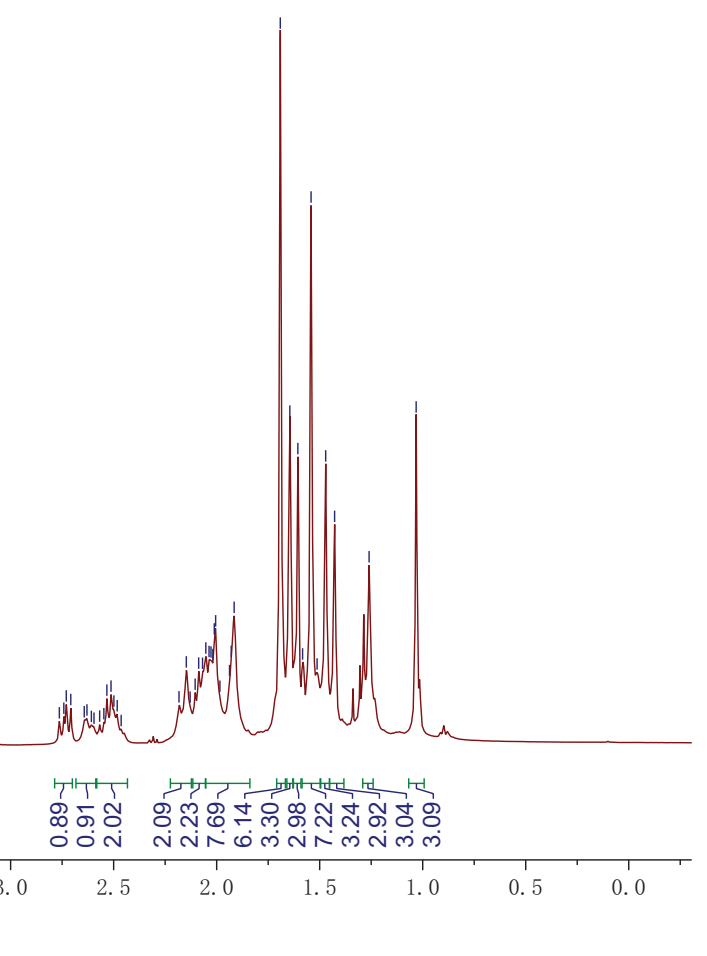


ம

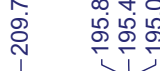

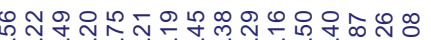

กิ่0ํำ

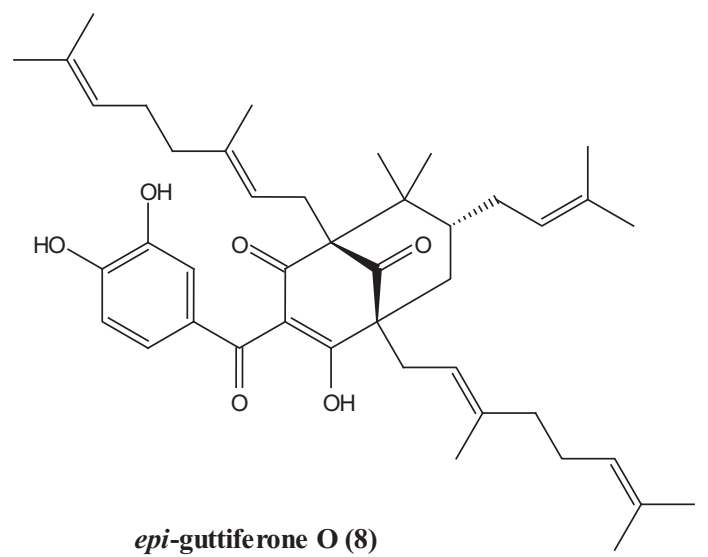

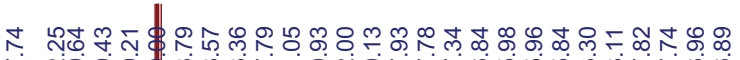

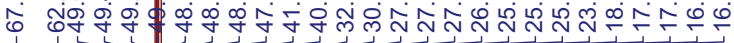

$\mid$

\begin{tabular}{llllllllllllll}
\hline 30 & 220 & 210 & 200 & 190 & 180 & 170 & 160 & 150 & 140 & 130 & 120 & 110 & 100 \\
$\mathrm{f} 1$ & $(\mathrm{ppm})$
\end{tabular}

$90 \quad 80 \quad 70 \quad 60$

$50 \quad 40$

20 